hep-th/0212188

YITP-02-62

ITFA-2002-39

December, 2002

\title{
Exact Description of D-branes via Tachyon Condensation
}

\author{
Tsuguhiko Asakawa ${ }^{1}$, Shigeki Sugimoto ${ }^{2}$ and Seiji Terashima ${ }^{3}$ \\ 1 Yukawa Institute for Theoretical Physics, Kyoto University, \\ Kyoto 606-8502, Japan \\ 2 The Niels Bohr Institute, \\ Blegdamsvej 17, DK-2100 Copenhagen Ø, Denmark \\ ${ }^{3}$ Institute for Theoretical Physics, University of Amsterdam \\ Valckenierstraat 65, 1018 XE, Amsterdam, The Netherlands
}

\begin{abstract}
We examine the fluctuations around a $\mathrm{D} p$-brane solution in an unstable D-brane system using boundary states and also boundary string field theory. We show that the fluctuations correctly reproduce the fields on the $\mathrm{D} p$-brane. Plugging these into the action of the unstable D-brane system, we recover not only the tension and RR charge, but also full effective action of the D $p$-brane exactly. Our method works for general unstable D-brane systems and provides a simple proof of D-brane descent/ascent relations under the tachyon condensation. In the lowest dimensional unstable D-brane system, called K-matrix theory, D-branes are described in terms of operator algebra. We show the equivalence of the geometric and algebraic descriptions of a D-brane world-volume manifold using the equivalence between path integral and operator formulation of the boundary quantum mechanics. As a corollary, the Atiyah-Singer index theorem is naturally obtained by looking at the coupling to RR-fields. We also generalize the argument to type I string theory.
\end{abstract}

\footnotetext{
${ }^{1}$ E-mail: asakawa@yukawa.kyoto-u.ac.jp

${ }^{2} \mathrm{E}$-mail: sugimoto@nbi.dk

${ }^{3}$ E-mail: sterashi@science.uva.nl
} 


\section{Contents}

1 Introduction $\quad 2$

2 Boundary states and BSFT action $\quad 4$

3 D-branes in K-matrix theory $\quad 11$

3.1 K-matrix theory and D-brane solutions . . . . . . . . . . . . . 11

3.2 The gauge field . . . . . . . . . . . . . . . . . 13

3.3 The scalar fields . . . . . . . . . . . . . . . . . 16

3.4 The tachyon field . . . . . . . . . . . . . . . 17

3.5 Superfield formulation and general fluctuations . . . . . . . . . . . 18

3.6 CS-term and the index theorem . . . . . . . . . . . . . . . . 23

3.6.1 General CS-terms . . . . . . . . . . . . . . . . 24

3.6.2 Ascent relations of CS-terms . . . . . . . . . . . . 27

3.6.3 An interpretation of the index theorem . . . . . . . . . 28

3.7 Application to non-commutative gauge theory . . . . . . . . . . 33

4 Generalization to higher dimensional systems $\quad 34$

4.1 D-brane descent relations . . . . . . . . . . . . . . . . . 35

$4.2 \mathrm{D} p$-branes from unstable $\mathrm{D} q$-brane systems . . . . . . . . . . . . . 36

$5 \quad$ Generalization to type I string theory $\quad 38$

5.1 Hidden Clifford algebra structure . . . . . . . . . . . . . . 38

5.2 D-branes in type I K-matrix theory . . . . . . . . . . . . . . . . 41

5.3 CS-term, real superconnections and the index theorem . . . . . . . . 44

6 Conclusions and Discussions $\quad 47$

$\begin{array}{ll}\text { A Operator vs. path integral } & 48\end{array}$

B Normalization $\quad 52$

C A-roof genus in CS-term 


\section{Introduction}

A D-brane was first introduced in [1] as an extended object on which the end points of open strings can be attached. Thanks to the duality between open and closed strings, it can also be seen as a source of closed strings, which is described by a boundary state $[2,3]$. On the other hand, D-branes are constructed as non-commutative configurations in matrix models [4]. More recently, D-branes are realized as solitons in the gauge theory with tachyon fields defined on higher dimensional unstable D-brane systems [5]. The relation between the higher dimensional unstable D-brane system and the lower dimensional D-brane soliton is called D-brane descent relation. From this construction, it has been revealed that the D-brane charges are classified by K-theory [6, 7, 8]. Analogously, it has been shown in $[9,10,11]$ that D-branes can also be constructed as bound states of a lower dimensional unstable D-brane system. We call this relation as D-brane ascent relation. In [11], construction of D-branes from a matrix theory based on an unstable D-instanton or D-particle system, which we call K-matrix theory, is investigated in detail. In particular, it has been shown that the classification of the D-branes is naturally given by analytic K-homology, which is consistent with the Ktheory result mentioned above. Note that in the latter two approaches, the tachyon condensation ${ }^{4}$ plays a crucial role in the construction of D-branes.

In this paper, we will make the equivalence among these different descriptions of D-branes transparent. The basic idea is as follows. We start with a boundary state representing an unstable D-brane system, and turn on the boundary interaction which represents the $\mathrm{D} p$-brane solution. Then, we show that the resulting boundary state is nothing but the boundary state of the $\mathrm{D} p$-brane. This strategy has been taken in several works. In $[5,13]$, the boundary states with non-trivial tachyon configurations are considered in some special cases in which the boundary interaction become exactly marginal operators. In [14], non-commutative D-branes are constructed by turning on non-commutative configurations of scalar fields in the boundary state representing infinitely many D-instantons. In [11], this strategy is used to construct D-brane boundary states from the D-brane solutions in K-matrix theory. One of the purposes

\footnotetext{
${ }^{4}$ For early works on tachyon condensation in string theory, see [12].
} 
of this paper is to extend the program given in [11] to include fluctuations around the D-brane solution. We will show that the fluctuations around the D-brane solution correctly reproduce the fields on the D-brane. We allow the fluctuations to be off-shell so that we can apply the results to the boundary string field theory (BSFT) [15]-[21]. 5 It immediately follows that the effective action for the fluctuations around the D-brane solution precisely reproduces the BSFT action for the D-brane. Therefore, we recover not only the tension and RR charge, but also full effective action of the $\mathrm{D} p$-brane exactly. Since the boundary state contains all the information about the D-brane, we think this provides a formal proof of the D-brane descent/ascent relations. Furthermore, we will develop a superfield formulation of the boundary interaction, which was introduced in [23]. The derivation of the D-brane descent/ascent relations is drastically simplified by utilizing this superfield formulation.

Although our method works for general unstable D-brane systems, we demonstrate it by mainly dealing with the lowest dimensional unstable D-brane system, i.e. the K-matrix theory. K-matrix theory is the simplest set up which can realize any Dbrane configurations. We consider a system with infinitely many non-BPS D-instantons (for type IIA or type I) or D-instanton - anti D-instanton pairs (for type IIB). Thus the matrix variables in the theory are considered as linear operators acting on an infinite dimensional Hilbert space. D-branes are represented algebraically by using these operators. Actually, as it has been shown in [11], a D-brane is realized as (a limit of) a spectral triple, which is introduced by Connes as a realization of Riemannian manifold in non-commutative geometry [24]. This observation makes it possible to naturally realize a D-brane whose world-volume is a non-commutative space. We will repeatedly see that the equivalence of the geometric and algebraic descriptions of a Dbrane world-volume manifold follows from the equivalence between operator formalism and path integral formalism of boundary quantum mechanics $[14,11]$. It is quite interesting that we can understand the correspondence between operator algebra and geometry, which is the starting point for the non-commutative geometry, using string theory. Another application for the correspondence between geometric and algebraic descriptions is the Atiyah-Singer index theorem, which relates the index of a Dirac

\footnotetext{
${ }^{5}$ Similar approach can also be found in [22].
} 
operator with the Chern number of the gauge bundle. We will give a direct physical interpretation for this relation by examining the coupling to RR-fields.

We also generalize the argument to type I string theory. The hidden real Clifford algebra structure of the Chan-Paton Hilbert space for type I unstable D-brane systems found in [25] plays a crucial role. We will see that the Chern-Simons terms for type I D-branes can be written down by using real superconnections.

The paper is organized as follows. In section 2, we review the boundary states with boundary interactions and the BSFT action for unstable D-brane systems. Section 3 is devoted to the construction of D-branes in K-matrix theory. We show that the fluctuations around a $\mathrm{D} p$-brane solution in K-matrix theory correctly reproduce the fields on the $\mathrm{D} p$-brane. The $\mathrm{D} p$-brane boundary states with the boundary interactions are precisely reproduced from the boundary state of unstable D-instanton system. We generalize this argument to higher dimensional systems in section 4 . In particular, the decent relations of D-branes are shown in terms of the boundary states. Section 5 deals with the generalization to Type I string theory. Finally we discuss further application and some speculative discussions in section 6 .

\section{Boundary states and BSFT action}

In this section we review the boundary states and the BSFT action for unstable Dbrane systems in type II string theory. The D-brane boundary states are obtained as linear combinations of the boundary states defined by [2] ${ }^{6}$

$$
|B p ; \pm\rangle=\int\left[d x^{\alpha}\right]\left[d \psi^{\alpha}\right]\left|x^{\alpha}, x^{i}=0\right\rangle\left|\psi^{\alpha}, \psi^{i}=0 ; \pm\right\rangle
$$

where the superscript $\alpha$ and $i$ represent the direction tangent and transverse to the D-brane, respectively. The state $|x\rangle$ and $|\psi\rangle$ are the coherent states satisfying

$$
\begin{aligned}
X^{\mu}(\sigma)|x\rangle & =x^{\mu}(\sigma)|x\rangle, \\
\Psi_{ \pm}^{\mu}(\sigma)|\psi ; \pm\rangle & =\psi^{\mu}(\sigma)|\psi ; \pm\rangle,
\end{aligned}
$$

\footnotetext{
${ }^{6}$ We omit the ghost part which does not play any role in this paper. See for example [3].
} 
where $^{7}$

$$
\begin{aligned}
X^{\mu}(\sigma) & =\widehat{x}_{0}^{\mu}+i \sum_{m \neq 0}\left(\frac{\alpha_{m}^{\mu}}{m} e^{-i m \sigma}+\frac{\widetilde{\alpha}_{m}^{\mu}}{m} e^{i m \sigma}\right), \\
\Psi_{ \pm}^{\mu}(\sigma) & =\sum_{r}\left(\Psi_{r}^{\mu} e^{-i r \sigma} \pm i \widetilde{\Psi}_{r}^{\mu} e^{i r \sigma}\right)
\end{aligned}
$$

are NS-R closed string operators at the boundary of the world-sheet, which act on the closed string Hilbert space, and $x^{\mu}(\sigma)$ and $\psi^{\mu}(\sigma)$ are bosonic and fermionic functions on $S^{1}(0 \leq \sigma \leq 2 \pi)$, respectively.

The explicit form of the coherent states are given as follows.

$$
\begin{aligned}
|x\rangle & =\exp \left\{\sum_{m=1}^{\infty}\left(-\frac{1}{2} x_{-m} x_{m}-a_{m}^{\dagger} \widetilde{a}_{m}^{\dagger}+a_{m}^{\dagger} x_{m}+x_{-m} \widetilde{a}_{m}^{\dagger}\right)\right\}\left|x_{0}\right\rangle, \\
|\psi ; \pm\rangle & =\exp \left\{\sum_{r>0}\left(-\frac{1}{2} \psi_{-r} \psi_{r} \pm i \Psi_{r}^{\dagger} \widetilde{\Psi}_{r}^{\dagger}+\Psi_{r}^{\dagger} \psi_{r} \mp i \psi_{-r} \widetilde{\Psi}_{r}^{\dagger}\right)\right\}|0\rangle,
\end{aligned}
$$

where

$$
a_{m}^{\mu}=i \alpha_{m}^{\mu} / \sqrt{m}, \quad a_{-m}^{\mu}=a_{m}^{\mu \dagger}=-i \alpha_{-m}^{\mu} / \sqrt{m}, \quad(m>0)
$$

and the $x_{m}$ and $\psi_{r}$ are the Fourier coefficients of the eigen functions in (2.2) and (2.3);

$$
x^{\mu}(\sigma)=x_{0}^{\mu}+\sum_{m \neq 0} \frac{1}{\sqrt{|m|}} x_{m}^{\mu} e^{-i m \sigma}, \quad \psi^{\mu}(\sigma)=\sum_{r} \psi_{r}^{\mu} e^{-i r \sigma} .
$$

The state $\left|x_{0}\right\rangle$ in (2.6) is the eigen state of the zero mode $\widehat{x}_{0}^{\mu}$ in (2.4). The equation (2.7) is for the NSNS-sector. For the RR-sector, we should replace $|0\rangle$ with the eigen state $\left|\psi_{0} ; \pm\right\rangle$ for the fermion zero modes $\Psi_{0 \pm}^{\mu}=\Psi_{0}^{\mu} \pm i \widetilde{\Psi}_{0}^{\mu}$. See [11] for our convention.

Fields on the D-brane world-volume can be turned on through boundary interaction. Then, the boundary states $|B p ; \pm\rangle$ are modified as

$$
\begin{aligned}
|B p ; \pm\rangle_{S_{b}} & =e^{-S_{b}\left(X, \Psi_{ \pm}\right)}|B p ; \pm\rangle \\
& =\int\left[d x^{\alpha}\right]\left[d \psi^{\alpha}\right] e^{-S_{b}(x, \psi)}\left|x^{\alpha}, x^{i}=0\right\rangle\left|\psi^{\alpha}, \psi^{i}=0 ; \pm\right\rangle
\end{aligned}
$$

where $S_{b}$ is the boundary action which represent the boundary interaction. We often omit the subscript \pm of the fermion $\Psi_{ \pm}^{\mu}$ in the following. The boundary interaction

\footnotetext{
${ }^{7}$ In this paper, we set $\alpha^{\prime}=2$, unless we recover explicit $\alpha^{\prime}$ dependence.
} 
for the gauge fields on the D-brane is well-known [2] and given as a supersymmetric generalization of Wilson loop operator

$$
e^{-S_{b}(X, \Psi)}=\operatorname{Tr} \mathrm{P} \exp \left\{-\int d \sigma\left(A_{\alpha}(X) \dot{X}^{\alpha}-\frac{1}{2} F_{\alpha \beta}(X) \Psi^{\alpha} \Psi^{\beta}\right)\right\} .
$$

Here, the gauge field $A_{\alpha}$ is taken to be anti-hermitian matrix.

It is useful to write down this boundary state using superfields. Let us introduce superfields $\mathbf{X}^{\mu}(\widehat{\sigma})=X^{\mu}(\sigma)+i \theta \Psi^{\mu}(\sigma)$ and $\mathbf{x}^{\mu}(\widehat{\sigma})=x^{\mu}(\sigma)+i \theta \psi^{\mu}(\sigma)$, where $\widehat{\sigma}=(\sigma, \theta)$ is the boundary supercoordinate. The covariant derivative is defined as $D=\partial_{\theta}+\theta \partial_{\sigma}$. Then, the boundary interaction (2.12) can be written as [23]

$$
e^{-S_{b}(X, \Psi)}=\operatorname{Tr} \widehat{\mathrm{P}} \exp \left(-\int d \widehat{\sigma} A_{\alpha}(\mathbf{X}) D \mathbf{X}^{\alpha}\right)
$$

where $d \widehat{\sigma}=d \sigma d \theta$. Here $\widehat{\mathrm{P}}$ denotes the supersymmetric path ordered product, which is defined as

$$
\begin{aligned}
& \widehat{\mathrm{P}} \exp \left(\int d \hat{\sigma} \mathbf{M}(\hat{\sigma})\right) \\
= & \sum_{n=0}^{\infty}(-1)^{\frac{n(n-1)}{2}} \int d \widehat{\sigma}_{1} \cdots d \widehat{\sigma}_{n} \Theta\left(\widehat{\sigma}_{12}\right) \Theta\left(\widehat{\sigma}_{23}\right) \cdots \Theta\left(\widehat{\sigma}_{n-1 n}\right) \mathbf{M}\left(\widehat{\sigma}_{1}\right) \cdots \mathbf{M}\left(\widehat{\sigma}_{n}\right),
\end{aligned}
$$

where $\widehat{\sigma}_{a b}=\sigma_{a}-\sigma_{b}-\theta_{a} \theta_{b}$ and $\Theta$ is a step function. If we perform the $d \theta$ integral in (2.14), we obtain the ordinary path ordered product as

$$
\widehat{\mathrm{P}} e^{\int d \hat{\sigma} \mathbf{M}(\hat{\sigma})}=\mathrm{P} e^{\int d \sigma\left(M_{1}-M_{0}^{2}\right)(\sigma)},
$$

where we write $\mathbf{M}(\widehat{\sigma})=M_{0}(\sigma)+\theta M_{1}(\sigma)$. Note that $M_{0}^{2}$ term in (2.15) comes from the contact $\delta$-function part of the expansion $\Theta\left(\widehat{\sigma}_{a b}\right)=\Theta\left(\sigma_{a}-\sigma_{b}\right)-\theta_{a} \theta_{b} \delta\left(\sigma_{a}-\sigma_{b}\right)$. Using this formula (2.15), it is easy to recover (2.12) from (2.13).

The boundary interactions for space-time filling unstable D-brane systems (nonBPS D9-branes in type IIA and D9- $\overline{\mathrm{D} 9}$ system in type IIB), which include gauge fields and tachyon fields, are obtained in [26, 18, 20, 21]. It is straightforward to generalize their argument to lower dimensional D-brane systems as described below.

First, we introduce a matrix consists of a condensate of the open string vertices in the superfield notation

$$
\mathbf{M}=\left(\begin{array}{cc}
-A_{\alpha}(\mathbf{X}) D \mathbf{X}^{\alpha}-i \Phi^{i}(\mathbf{X}) \mathbf{P}_{i} & T(\mathbf{X}) \\
T(\mathbf{X})^{\dagger} & -\widetilde{A}_{\alpha}(\mathbf{X}) D \mathbf{X}^{\alpha}-i \widetilde{\Phi}^{i}(\mathbf{X}) \mathbf{P}_{i}
\end{array}\right)
$$


where $\mathbf{P}_{i}(\widehat{\sigma})=\theta P_{i}(\sigma)+i \Pi_{i}(\sigma)$. Here $P_{i}(\sigma)$ and $\Pi_{i}(\sigma)$ are the conjugate momenta of $X^{i}(\sigma)$ and $\Psi^{i}(\sigma)$, respectively. $A_{\alpha}, \widetilde{A}_{\alpha}, \Phi^{i}, \widetilde{\Phi}^{i}$ and $T$ are the fields on the D-brane. 8 These fields are independent for $\mathrm{D} p-\overline{\mathrm{D} p}$-brane system. Namely, $A_{\alpha}$ and $\Phi^{i}$ are the gauge field and scalar fields on the $\mathrm{D} p$-branes, $\widetilde{A}_{\mu}$ and $\widetilde{\Phi}^{i}$ are those on the $\overline{\mathrm{D} p}$-branes and $T$ is the tachyon field which is created by the open string stretched between the $\mathrm{D} p$-branes and the $\overline{\mathrm{D} p}$-branes. For non-BPS D $p$-brane, we have constraints $A_{\alpha}=\widetilde{A}_{\alpha}$, $\Phi^{i}=\widetilde{\Phi}^{i}$ and $T^{\dagger}=T$.

Let us consider $N$ pairs of $\mathrm{D} p$-brane and $\overline{\mathrm{D} p}$-brane. We can decompose the matrix (2.16) using Pauli matrices $\sigma_{1}$ and $\sigma_{2}$ as

$$
\begin{aligned}
\mathbf{M}=- & \left(A_{\alpha}^{+} D \mathbf{X}^{\alpha}+i \Phi_{+}^{i} \mathbf{P}_{i}\right) \otimes 1_{2}-\left(A_{\alpha}^{-} D \mathbf{X}^{\alpha}+i \Phi_{-}^{i} \mathbf{P}_{i}\right) \otimes i \sigma_{2} \sigma_{1} \\
& +T_{1} \otimes \sigma_{1}+T_{2} \otimes \sigma_{2},
\end{aligned}
$$

where $A_{\alpha}^{ \pm}=\frac{1}{2}\left(A_{\alpha} \pm \widetilde{A}_{\alpha}\right), \Phi_{ \pm}^{i}=\frac{1}{2}\left(\Phi^{i} \pm \widetilde{\Phi}^{i}\right), T_{1}=\frac{1}{2}\left(T+T^{\dagger}\right)$ and $T_{2}=\frac{i}{2}\left(T-T^{\dagger}\right)$. Then, the boundary interaction can be written as

$$
\begin{aligned}
e^{-S_{b}}=\int\left[d \boldsymbol{\Gamma}^{1}\right] & \left.d d \boldsymbol{\Gamma}^{2}\right] \operatorname{Tr} \widehat{\mathrm{P}} \exp \left\{\int d \widehat { \sigma } \left(\frac{1}{4} \boldsymbol{\Gamma}^{1} D \boldsymbol{\Gamma}^{1}+\frac{1}{4} \boldsymbol{\Gamma}^{2} D \boldsymbol{\Gamma}^{2}\right.\right. \\
- & \left(A_{\alpha}^{+} D \mathbf{X}^{\alpha}+i \Phi_{+}^{i} \mathbf{P}_{i}\right)-\left(A_{\alpha}^{-} D \mathbf{X}^{\alpha}+i \Phi_{-}^{i} \mathbf{P}_{i}\right) i \boldsymbol{\Gamma}^{2} \boldsymbol{\Gamma}^{1} \\
& \left.\left.+T_{1} \boldsymbol{\Gamma}^{1}+T_{2} \boldsymbol{\Gamma}^{2}\right)\right\},
\end{aligned}
$$

where $\Gamma^{I}(\widehat{\sigma})=\eta^{I}(\sigma)+\theta F^{I}(\sigma)(I=1,2)$ are real fermionic superfields. Here the trace Tr is taken over remaining $N$ Chan-Paton indices. The basic idea to obtain (2.18) from the matrix (2.17) is to replace the Pauli matrices $\sigma^{I}$ with fields $\eta^{I}$ which satisfy the same algebra as $\sigma^{I}$, i.e.

$$
\left\{\widehat{\eta}^{I}, \widehat{\eta}^{I}\right\}=2 \delta^{I J}
$$

in the operator formulation and combine them with their superpartner fields $F^{I}$ in the superfields $\Gamma^{I}$.

This prescription can easily be generalized to the case in which the matrix $\mathbf{M}$ is expanded by $S O(2 m)$ gamma matrices $\Gamma^{I}=\left({ }_{\gamma^{I \dagger}} \gamma^{I}\right)(I=1, \ldots, 2 m)$ as

$$
\mathbf{M}=\sum_{k=0}^{2 m} \mathbf{M}^{I_{1} \cdots I_{k}} \otimes \Gamma^{I_{1} \cdots I_{k}},
$$

\footnotetext{
${ }^{8}$ We do not consider massive modes and fermions in this paper.
} 
where $\Gamma^{I_{1} \cdots I_{k}}$ denote the skew-symmetric products of the gamma matrices. In this case, the boundary interaction becomes [20]

$$
e^{-S_{b}}=\int\left[d \boldsymbol{\Gamma}^{I}\right] \operatorname{Tr} \widehat{\mathrm{P}} \exp \left\{\int d \widehat{\sigma}\left(\frac{1}{4} \boldsymbol{\Gamma}^{I} D \boldsymbol{\Gamma}^{I}+\sum_{k=0}^{2 m} \mathbf{M}^{I_{1} \cdots I_{k}} \boldsymbol{\Gamma}^{I_{1}} \cdots \boldsymbol{\Gamma}^{I_{k}}\right)\right\} .
$$

Here, the path ordered product and the trace $\operatorname{Tr} \mathrm{P}$ is needed when $\mathbf{M}^{I_{1} \ldots I_{k}}$ are still matrices. Note that $F^{I}$ are auxiliary fields which can be integrated out. After performing $\theta$ integral and integrating out the auxiliary fields $F^{I}$, we obtain [20]

$$
e^{-S_{b}}=\int\left[d \eta^{I}\right] \operatorname{Tr} \mathrm{P} \exp \left\{\int d \sigma\left(\frac{1}{4} \dot{\eta}^{I} \eta^{I}+\sum_{k=0}^{2 m} M^{I_{1} \cdots I_{k}} \eta^{I_{1}} \cdots \eta^{I_{k}}\right)\right\},
$$

where

$$
\begin{gathered}
M=\hat{\mathcal{A}}+\hat{\mathcal{F}} \\
=\sum_{k=0}^{2 m} M^{I_{1} \cdots I_{k}} \otimes \Gamma^{I_{1} \cdots I_{k}}, \\
\hat{\mathcal{A}}=\left(\begin{array}{cc}
\mathcal{A} & \\
\widetilde{\mathcal{A}}
\end{array}\right), \\
\hat{\mathcal{F}}=\left(\begin{array}{cc}
-T T^{\dagger}+\mathcal{F} & \mathcal{D} T \\
\mathcal{D} T^{\dagger} & -T^{\dagger} T+\widetilde{\mathcal{F}}
\end{array}\right), \\
\mathcal{\mathcal { A }}=-A_{\alpha} \dot{X}^{\alpha}-i \Phi^{i} P_{i} \\
\mathcal{\mathcal { F }}=\frac{1}{2} F_{\alpha \beta} \Psi^{\alpha} \Psi^{\beta}+i\left(\partial_{\alpha} \Phi^{i}+\left[A_{\alpha}, \Phi^{i}\right]\right) \Psi^{\alpha} \Pi_{i}-\frac{1}{2}\left[\Phi^{i}, \Phi^{j}\right] \Pi_{i} \Pi_{j} \\
\widetilde{\mathcal{F}}=\frac{1}{2} \widetilde{F}_{\alpha \beta} \Psi^{\alpha} \Psi^{\beta}+i\left(\partial_{\alpha} \widetilde{\Phi}^{i}+\left[\widetilde{A}_{\alpha}, \widetilde{\Phi}^{i}\right]\right) \Psi^{\alpha} \Pi_{i}-\frac{1}{2}\left[\widetilde{\Phi}^{i}, \widetilde{\Phi}^{j}\right] \Pi_{i} \Pi_{j} \\
\mathcal{D} T=i\left(\partial_{\alpha} T+A_{\alpha} T-T \widetilde{A}_{\alpha}\right) \Psi^{\alpha}-\left(\Phi^{i} T-T \widetilde{\Phi}^{i}\right) \Pi_{i} \\
\mathcal{D} T^{\dagger}=i\left(\partial_{\alpha} T^{\dagger}+\widetilde{A}_{\alpha} T^{\dagger}-T^{\dagger} A_{\alpha}\right) \Psi^{\alpha}-\left(\widetilde{\Phi}^{i} T^{\dagger}-T^{\dagger} \Phi^{i}\right) \Pi_{i} .
\end{gathered}
$$

It is also useful to note that $\widehat{\mathcal{F}}$ can be written as

$$
\widehat{\mathcal{F}}=-Z^{2}, \quad Z=\left(\begin{array}{cc}
-i \mathcal{D} & T \\
T^{\dagger} & -i \widetilde{\mathcal{D}}
\end{array}\right)
$$

where $\mathcal{D}=\Psi^{\alpha}\left(\partial_{\alpha}+A_{\alpha}\right)+i \Pi_{i} \Phi^{i}$ and $\widetilde{\mathcal{D}}=\Psi^{\alpha}\left(\partial_{\alpha}+\widetilde{A}_{\alpha}\right)+i \Pi_{i} \widetilde{\Phi}^{i}$. 
If we further perform the integral over $\eta^{I}$, the boundary interaction (2.22) becomes

$$
e^{-S_{b}}=\left\{\begin{array}{cl}
\kappa \operatorname{Tr} \mathrm{P} e^{\int d \sigma M(\sigma)} & \text { (NS-NS sector), } \\
\operatorname{Str} \mathrm{P} e^{\int d \sigma M(\sigma)} & \text { (R-R sector) }
\end{array}\right.
$$

where $M$ is given as (2.23). The normalizaton constant $\kappa$ is $\kappa=1$ for $\mathrm{D} p-\overline{\mathrm{D} p}$ systems and $\kappa=1 / \sqrt{2}$ for non-BPS D-branes. See Appendix A and B for the derivation. Note that, in computing the products of the matrix $M, \Gamma^{I}$ in the gamma matrix expansion (2.24) are treated as fermionic gamma matrices, which anti-commute with $\Psi^{\mu}$ and $\Pi_{\mu}$, since $\eta^{I}$ are fermionic. (See [20].) This formula (2.34) reduces to (2.12) when we only turn on the gauge field $A_{\alpha}$. Here Str denote the supertrace defined as $\operatorname{Str}(\cdots)=\kappa \operatorname{Tr}(\Gamma \cdots)$, where $\Gamma=(-i)^{n / 2} \prod_{I=1}^{n} \Gamma^{I}$ is the skew-symmetric product of all the gamma matrices $\Gamma^{I}(I=1, \ldots, n)$ which take place in the gamma matrix expansion. 9 The difference between NS-NS sector and R-R sector comes from the boundary condition of the boundary fermion fields. In the R-R sector, $\eta^{I}(\sigma)$ are periodic with respect to $\sigma$ and there is a zero mode for each of them. Therefore, we have to saturate the zero modes in the path integral (2.22), implying the supertrace rather than the usual trace.

For the $\mathrm{D} p-\overline{\mathrm{D} p}$ system, we can see from $(2.23)-(2.26)$ that $e^{\int d \sigma M}$ is of the form

$$
\left(\begin{array}{ll}
\alpha & \beta \\
\gamma & \delta
\end{array}\right),
$$

where $\alpha, \beta, \gamma$ and $\delta$ are the components related to $\mathrm{D} p$ - $\mathrm{D} p$ string, $\mathrm{D} p-\overline{\mathrm{D} p}$ string, $\overline{\mathrm{D} p}-\mathrm{D} p$ string and $\overline{\mathrm{D} p}-\overline{\mathrm{D} p}$ string, respectively. This is also the case for non-BPS D-branes, if we impose the constraints $\alpha=\delta$ and $\beta=\gamma$. Thus this matrix can be expanded with respect to $\sigma_{1}$ and $\sigma_{2}$ for the $\mathrm{D} p-\overline{\mathrm{D} p}$ system, just as we did in (2.17), while only $\sigma_{1}$ is needed for the non-BPS D-branes. Therefore, we have

$$
\begin{aligned}
& \operatorname{Str}\left(\begin{array}{cc}
\alpha & \beta \\
\gamma & \delta
\end{array}\right)=\left\{\begin{array}{cl}
\operatorname{Tr}\left[(-i) \sigma_{1} \sigma_{2}\left(\begin{array}{c}
\alpha \beta \\
\gamma \delta
\end{array}\right)\right], & (\mathrm{D} p \text { - } \overline{\mathrm{D} p} \text { system }), \\
\frac{1}{\sqrt{2}} \operatorname{Tr}\left[(-i)^{1 / 2} \sigma_{1}\left(\begin{array}{c}
\alpha \beta \\
\beta \alpha
\end{array}\right)\right], & \text { (non-BPS D } p \text {-branes }),
\end{array}\right. \\
& =\left\{\begin{array}{cl}
\operatorname{tr} \alpha-\operatorname{tr} \delta, & (\mathrm{D} p-\overline{\mathrm{D} p} \text { system }), \\
(-2 i)^{1 / 2} \operatorname{tr} \beta, & \text { (non-BPS D } p \text {-branes). }
\end{array}\right.
\end{aligned}
$$

\footnotetext{
${ }^{9}$ Note that the normalization of the supertrace defined here is slightly different from the usual one when $n$ is odd, i.e. for the non-BPS D-branes. We adopt this normalization in order to treat the $\mathrm{D} p-\overline{\mathrm{D} p}$ system and non-BPS $\mathrm{D} p$-branes in the same notation.
} 
The formula (2.34) together with (2.37) can also be used in the case with different numbers of $\mathrm{D} p$-branes and $\overline{\mathrm{D} p}$-branes. In particular, we can consider BPS $\mathrm{D} p$-branes by setting the size of the matrices $\beta, \gamma$ and $\delta$ to be zero, which again reproduces (2.12).

Note also that (2.23) is given as $M=M_{1}-M_{0}^{2}$, where $\mathbf{M}=M_{0}+\theta M_{1}$ is the matrix in (2.16), and hence the boundary interaction (2.34) can also be written in superfields as

$$
e^{-S_{b}}=\left\{\begin{array}{cl}
\kappa \operatorname{Tr} \widehat{\mathrm{P}} e^{\int d \hat{\sigma} \mathbf{M}(\hat{\sigma})} & (\mathrm{NS}-\mathrm{NS} \text { sector }) \\
\mathrm{Str} \widehat{\mathrm{P}} e^{\int d \hat{\sigma} \mathbf{M}(\hat{\sigma})} & (\mathrm{R}-\mathrm{R} \text { sector })
\end{array}\right.
$$

using the relation (2.15). See Appendix A for a direct derivation of the equivalence between (2.21) and (2.38). To sum up, we can use any one of (2.21), (2.22), (2.34) and (2.38) as the boundary interaction.

The boundary state for a $\mathrm{D} p$-brane is given by a linear combination of $|B p ; \pm\rangle$ with the boundary interaction such that

$$
|D p\rangle_{S_{b}}=P \widetilde{P}_{+} e^{-S_{b}}|B p ;+\rangle_{\mathrm{NS}}+P \widetilde{P}_{ \pm} e^{-S_{b}}|B p ;+\rangle_{\mathrm{RR}}
$$

where $P=\frac{1}{2}\left(1+(-1)^{F}\right)$ and $\widetilde{P}_{ \pm}=\frac{1}{2}\left(1 \pm(-1)^{\widetilde{F}}\right)$ are GSO projection operators. The subscript of $\widetilde{P}_{ \pm}$in the right hand side of (2.39) is + or - for type IIB or type IIA string theory, respectively.

The boundary string field theory (BSFT) action for the Dp-brane in superstring theory is given in $[18,20,21]$ as a disk partition function with the boundary interaction, which can be written as

$$
S\left(T, A_{\alpha}, \Phi^{i}, \cdots\right)=\frac{2 \pi}{g_{s}}\left\langle 0\left|e^{-S_{b}}\right| B p ;+\right\rangle_{\mathrm{NS}},
$$

using the NS-NS sector boundary state with the boundary interaction (2.10). ${ }^{10}$ Similarly the CS-term of the system is given by

$$
S_{C S}\left(C, T, A_{\alpha}, \Phi^{i}, \cdots\right)=\left\langle C\left|e^{-S_{b}}\right| B p ;+\right\rangle_{\mathrm{RR}}
$$

where $\langle C|$ is the state corresponding to the R-R fields.

There are many attempts to calculate the action (2.40) or the CS-term (2.41) explicitly. When the tachyon is absent, up to four derivative corrections to the DBI

\footnotetext{
${ }^{10}$ The normalization factor is determined in Appendix B.
} 
action is calculated in [27] using the boundary state with the boundary interaction (2.13). The CS-term is examined using the expression (2.41) in [28] and its derivative corrections are also calculated in [27]. For the unstable D-brane systems, the action has been obtained explicitly by now only for constant $\partial_{\mu} T$ and $F_{\mu \nu}$ [29] or up to the order $\left(\alpha^{\prime}\right)^{2}[21]$. However, we stress here that the boundary action of the BSFT action is renormalizable if we consider the tachyon and gauge fields only. Thus the BSFT action is well-defined for those fields and we can in principle calculate all the higher derivative corrections using the expression (2.40).

\section{D-branes in K-matrix theory}

In this section, we consider the construction of D-branes in a matrix theory based on an unstable D-brane system, which we call K-matrix theory. There are many choices of the unstable D-brane system. The lowest dimensional ones are non-BPS D-instanton system in type IIA string theory or D-instanton - anti D-instanton system in type IIB string theory. If we want to avoid the formal Euclidean rotation to obtain the D-instantons, we could start with D0- $\overline{\mathrm{D} 0}$ system in type IIA string theory or non-BPS D0-brane system in type IIB string theory.

In this section, we will mainly use the matrix theory based on the non-BPS Dinstantons in type IIA string theory for simplicity. The generalization to other unstable D-brane systems is straightforward.

\subsection{K-matrix theory and D-brane solutions}

Let us consider the matrix theory based on non-BPS D-instanton system in type IIA string theory. The field contents and the action is obtained as the dimensional reduction of non-BPS D9-brane system. The $N$ non-BPS D9-brane system is a ten dimensional gauge theory with $U(N)$ gauge symmetry with a tachyon field. In this paper, we only consider low-lying bosonic fields, that is, the tachyon field $T$ and the gauge field $A_{\mu}$, both of which transform as the adjoint representation under the gauge group. The dimensional reduction of these fields gives the tachyon $T$ and ten scalar fields $\Phi^{\mu}$ $(\mu=0,1, \ldots, 9)$. They are $N \times N$ hermitian matrices. We take $N$ to be infinity and 
regard these matrices as operators acting on an infinite dimensional Hilbert space. The BSFT action (2.40) of the system is

$$
S\left(\Phi^{\mu}, T\right)=\frac{2 \pi}{g_{s}}\left\langle 0\left|e^{-S_{b}\left(\Phi^{\mu}, T\right)}\right| B(-1) ;+\right\rangle_{\mathrm{NS}},
$$

where the boundary interaction is given by (2.21) or (2.38) with

$$
\mathbf{M}=\left(\begin{array}{cc}
-i \Phi^{\mu} \mathbf{P}_{\mu} & T \\
T & -i \Phi^{\mu} \mathbf{P}_{\mu}
\end{array}\right)
$$

or, (2.22) or (2.34) with

$$
M=\left(\begin{array}{cc}
-i \Phi^{\mu} P_{\mu}-T^{2}-\frac{1}{2}\left[\Phi^{\mu}, \Phi^{\nu}\right] \Pi_{\mu} \Pi_{\nu} & -\left[\Phi^{\mu}, T\right] \Pi_{\mu} \\
-\left[\Phi^{\mu}, T\right] \Pi_{\mu} & -i \Phi^{\mu} P_{\mu}-T^{2}-\frac{1}{2}\left[\Phi^{\mu}, \Phi^{\nu}\right] \Pi_{\mu} \Pi_{\nu}
\end{array}\right) .
$$

As argued in [11], we can construct any D-brane configuration expected in type IIA string theory using this matrix theory. In fact, it has been shown in [11] that the D-brane configurations are given as (a limit of) spectral triples, which are analytic description of Riemannian manifold [24], and they are classified by K-homology, which is consistent with the K-theory classification of D-brane charges $[7,8]$.

More explicitly, a solution representing a $\mathrm{D} p$-brane extended along $x^{0}, \ldots, x^{p}$-directions is given by

$$
\begin{aligned}
T & =u \sum_{\alpha=0}^{p} \widehat{p}_{\alpha} \otimes \gamma^{\alpha} \\
\Phi^{\alpha} & =\widehat{x}^{\alpha} \otimes 1 \quad(\alpha=0, \ldots, p), \quad \Phi^{i}=0 \quad(i=p+1, \ldots, 9),
\end{aligned}
$$

where $\widehat{x}^{\alpha}$ and $\widehat{p}_{\alpha}$ are operators on a Hilbert space $\mathcal{H}$ satisfying

$$
\left[\widehat{x}^{\alpha}, \widehat{p}_{\beta}\right]=i \delta_{\beta}^{\alpha}
$$

and $\gamma^{\alpha}$ are gamma matrices represented as hermitian matrices. $u$ is a real parameter and this configuration becomes an exact solution in the limit $u \rightarrow \infty$ [9]. Since the eigen values of $\Phi^{\mu}$ represent the position of the non-BPS D-instantons, we can see from (3.5) that this configuration represent a $p+1$ dimensional object, which is interpreted as the $\mathrm{D} p$-brane world volume.

The fields on the $\mathrm{D} p$-brane are given by the fluctuation around this solution (3.4, 3.5). Our goal is to consider all the relevant fluctuations around this solution and 
identify them with the fields on the $\mathrm{D} p$-branes and then show that we can exactly reproduce the effective action of the $\mathrm{D} p$-brane (2.40). For this purpose, it suffices to show that the boundary state of the $\mathrm{D} p$-brane $e^{-S_{b}}|B p, \pm\rangle$ is reproduced by inserting the $\mathrm{D} p$-brane solution and its fluctuations into the boundary state of the non-BPS D-instantons $e^{-S_{b}\left(\Phi^{\mu}, T\right)}|B(-1), \pm\rangle$. Actually, since the boundary state carries all the information about the D-brane, this explicitly shows that the $\mathrm{D} p$-brane is constructed from the non-BPS D-instantons. We emphasize that we recover not only the tension, RR charge or tachyon mass etc, but also full effective action of the $\mathrm{D} p$-brane as well as its coupling to every closed string mode exactly.

We start by introducing the fluctuations one by one in section 3.2-3.4 to identify them with the fields on the $\mathrm{D} p$-brane. We shall analyze more general fluctuations in section 3.5 after developing a formulation using superfield. The analysis will become surprisingly simple using this superfield method. Hasty readers could skip section 3.23.4 and proceed to section 3.5. The following sections, section 3.6 and section 3.7, are devoted to some applications of our argument.

\subsection{The gauge field}

Let us first consider the fluctuation which corresponds to the gauge field on the $\mathrm{D} p$ brane. A solution representing $N$ D p-branes can be obtained by piling $N$ copies of a single $\mathrm{D} p$-brane solution $(3.4,3.5)$ as

$$
\begin{aligned}
T & =u \sum_{\alpha=0}^{p} \widehat{p}_{\alpha} \otimes 1_{N} \otimes \gamma^{\alpha} \\
\Phi^{\alpha} & =\widehat{x}^{\alpha} \otimes 1_{N} \otimes 1 \quad(\alpha=0, \ldots, p), \quad \Phi^{i}=0 \quad(i=p+1, \ldots, 9),
\end{aligned}
$$

Here, $T$ and $\Phi^{\mu}$ are operators acting on a Hilbert space $\mathcal{H} \otimes \mathbf{C}^{N} \otimes S$, where $S$ is the spinor space on which the gamma matrices $\gamma^{\alpha}$ are represented. The gauge symmetry of the system is given by the transformation

$$
T \rightarrow U T U^{-1}, \quad \Phi^{\mu} \rightarrow U \Phi^{\mu} U^{-1}
$$

with a unitary operator $U$. The global gauge symmetry of the $\mathrm{D} p$-brane is the part of the unitary symmetry which is unbroken in the configuration (3.7) and (3.8), namely, 
the $U(N)$ subgroup of the form $U=1 \otimes h \otimes 1(h \in U(N))$. The local gauge symmetry of the $\mathrm{D} p$-brane is obtained by allowing the $\widehat{x}$ dependence in the unitary transformation as $U=h(\widehat{x}) \otimes 1$, where $h(x)$ is a $U(N)$ valued function. The gauge field corresponding to this is introduced in the tachyon operator as

$$
T=u \sum_{\alpha=0}^{p}\left(\widehat{p}_{\alpha}-i A_{\alpha}(\widehat{x})\right) \otimes \gamma^{\alpha}
$$

in order to maintain the covariance under the local gauge transformation [11]. Here we abbreviated $\widehat{p}_{\alpha} \otimes 1_{N}$ as $\widehat{p}_{\alpha}$.

Inserting (3.10) and (3.8) into (3.3), we obtain

$$
\begin{aligned}
M & =\left(\begin{array}{cc}
-i \widehat{x}^{\alpha} P_{\alpha}-u^{2}\left(\widehat{p}_{\alpha}-i A_{\alpha}\right)^{2}+\frac{u^{2}}{2} F_{\alpha \beta} \gamma^{\alpha \beta} & -i u \gamma^{\alpha} \Pi_{\alpha} \\
-i u \gamma^{\alpha} \Pi_{\alpha} & -i \widehat{x}^{\alpha} P_{\alpha}-u^{2}\left(\widehat{p}_{\alpha}-i A_{\alpha}\right)^{2}+\frac{u^{2}}{2} F_{\alpha \beta} \gamma^{\alpha \beta}
\end{array}\right) \\
& =-\left(i \widehat{x}^{\alpha} P_{\alpha}+u^{2}\left(\widehat{p}_{\alpha}-i A_{\alpha}\right)^{2}\right) I-i u \Pi_{\alpha} \Gamma^{\alpha}+\frac{u^{2}}{2} F_{\alpha \beta} \Gamma^{\alpha \beta},
\end{aligned}
$$

where we have defined $\Gamma^{\alpha}=\left(\gamma_{\gamma^{\alpha}}^{\gamma^{\alpha}}\right)$. Here we adopt (2.22) for the boundary interaction. Then, we obtain

$$
\begin{aligned}
& e^{-S_{b}\left(\Phi^{\mu}, T\right)} \\
= & \int\left[\eta^{\alpha}\right] \operatorname{Tr} \mathrm{P} \exp \left\{\int d \sigma\left(\frac{1}{4} \dot{\eta}^{\alpha} \eta^{\alpha}-i \widehat{x}^{\alpha} P_{\alpha}-u^{2}\left(\widehat{p}_{\alpha}-i A_{\alpha}\right)^{2}-i u \Pi_{\alpha} \eta^{\alpha}+\frac{u^{2}}{2} F_{\alpha \beta} \eta^{\alpha} \eta^{\beta}\right)\right\} \\
= & \int\left[d \eta^{\alpha}\right] e^{\int d \sigma\left(\frac{1}{4} \eta^{\alpha} \dot{\eta}^{\alpha}-i u \Pi_{\alpha} \eta^{\alpha}\right)} \operatorname{Tr} \mathrm{P} e^{-i \int d \sigma H(\hat{p}, \hat{x})},
\end{aligned}
$$

where $\operatorname{Tr}$ denotes the trace over $\mathcal{H} \otimes \mathbf{C}^{N}$ and we defined $i H(\widehat{p}, \widehat{x})=u^{2}\left(\widehat{p}_{\alpha}-i A_{\alpha}(\widehat{x})\right)^{2}+$ $i \widehat{x}^{\alpha} P_{\alpha}-\frac{u^{2}}{2} F_{\alpha \beta}(\widehat{x}) \eta^{\alpha} \eta^{\beta}$. Since the last term in (3.15) is usual quantum mechanical partition function with Hamiltonian $H(\widehat{p}, \widehat{x})$, we can rewrite it in terms of the path integral formulation using the standard formula

$$
\operatorname{Tr} \mathrm{P} e^{-i \int d \sigma H(\hat{p}, \hat{x})}=\int[d x] \operatorname{tr} \mathrm{P} e^{i \int d \sigma L(\dot{x}, x)},
$$

where $\operatorname{tr}$ in the right hand side denotes the trace over $\mathbf{C}^{N}$, i.e., with respect to the gauge indices of the resulting $\mathrm{D} p$-branes. The Lagrangian $L(\dot{x}, x)$ is obtained by the Legendre transformation of the Hamiltonian $H(\widehat{p}, \widehat{x})$

$$
i L(\dot{x}, x)=-\frac{\dot{x}_{\alpha}^{2}}{4 u^{2}}-A_{\alpha}(x) \dot{x}^{\alpha}-i x^{\alpha} P_{\alpha}+\frac{u^{2}}{2} F_{\alpha \beta}(x) \eta^{\alpha} \eta^{\beta} .
$$


Thus we obtain

$$
\begin{gathered}
e^{-S_{b}\left(\Phi^{\mu}, T\right)} \\
=\int\left[d x^{\alpha}\right]\left[d \eta^{\alpha}\right] \operatorname{tr} \mathrm{P} \exp \left\{\int d \sigma \left(\frac{1}{4} \dot{\eta}^{\alpha} \eta^{\alpha}-\frac{\dot{x}_{\alpha}^{2}}{4 u^{2}}\right.\right. \\
\left.\left.-A_{\alpha}(x) \dot{x}^{\alpha}-i x^{\alpha} P_{\alpha}+\frac{u^{2}}{2} F_{\alpha \beta}(x) \eta^{\alpha} \eta^{\beta}-i u \Pi_{\alpha} \eta^{\alpha}\right)\right\} \\
=\int\left[d x^{\alpha}\right]\left[d \psi^{\alpha}\right] \operatorname{tr} \mathrm{P} \exp \left\{\int d \sigma \left(-\frac{1}{4 u^{2}}\left(\dot{x}_{\alpha}^{2}+\psi^{\alpha} \dot{\psi}^{\alpha}\right)\right.\right. \\
\left.\left.-A_{\alpha}(x) \dot{x}^{\alpha}+\frac{1}{2} F_{\alpha \beta}(x) \psi^{\alpha} \psi^{\beta}-i x^{\alpha} P_{\alpha}-i \Pi_{\alpha} \psi^{\alpha}\right)\right\},
\end{gathered}
$$

where $\psi^{\alpha}=u \eta^{\alpha}$. ${ }^{11}$ Taking the limit $u \rightarrow \infty$, we obtain ${ }^{12}$

$$
\begin{aligned}
& e^{-S_{b}\left(\Phi^{\mu}, T\right)} \\
\rightarrow & \int\left[d x^{\alpha}\right]\left[d \psi^{\alpha}\right] \operatorname{tr} \mathrm{P} \exp \left\{\int d \sigma\left(-A_{\alpha}(x) \dot{x}^{\alpha}+\frac{1}{2} F_{\alpha \beta}(x) \psi^{\alpha} \psi^{\beta}-i x^{\alpha} P_{\alpha}-i \Pi_{\alpha} \psi^{\alpha}\right)\right\}
\end{aligned}
$$

and the boundary state will become

$$
\begin{aligned}
& e^{-S_{b}\left(\Phi^{\mu}, T\right)}|B(-1) ; \pm\rangle \\
& \rightarrow \quad \int\left[d x^{\alpha}\right]\left[d \psi^{\alpha}\right] \operatorname{tr} \mathrm{P} \exp \left\{\int d \sigma \left(-A_{\alpha}(x) \dot{x}^{\alpha}+\frac{1}{2} F_{\alpha \beta}(x) \psi^{\alpha} \psi^{\beta}\right.\right. \\
&\left.\left.\quad-i x^{\alpha} P_{\alpha}-i \Pi_{\alpha} \psi^{\alpha}\right)\right\}\left|x^{\mu}=0\right\rangle\left|\psi^{\mu}=0 ; \pm\right\rangle \\
&=\int\left[d x^{\alpha}\right]\left[d \psi^{\alpha}\right] \operatorname{tr} \mathrm{P} \exp \left\{\int d \sigma\left(-A_{\alpha}(x) \dot{x}^{\alpha}+\frac{1}{2} F_{\alpha \beta}(x) \psi^{\alpha} \psi^{\beta}\right)\right\} \\
& \quad \times\left|x^{\alpha}, x^{i}=0\right\rangle\left|\psi^{\alpha}, \psi^{i}=0 ; \pm\right\rangle \\
&=e^{-S_{b}\left(A_{\alpha}\right)}|B p ; \pm\rangle,
\end{aligned}
$$

where

$$
e^{-S_{b}\left(A_{\alpha}\right)}=\operatorname{tr} \mathrm{P} \exp \left\{\int d \sigma\left(-A_{\alpha}(X) \dot{X}^{\alpha}+\frac{1}{2} F_{\alpha \beta}(X) \Psi^{\alpha} \Psi^{\beta}\right)\right\}
$$

(3.25) is nothing but the boundary state for D $p$-brane (2.10) with the boundary interaction (2.12).

\footnotetext{
${ }^{11}$ We can show $[d \eta]=\left[u d \psi^{\alpha}\right]=\left[d \psi^{\alpha}\right]$ using the zeta-function regularization $[11]$.

${ }^{12}$ To be more precise, we should regularize the path integral before taking the $u \rightarrow \infty$ limit. The following procedure can be justified by using zeta-function regularization [11].
} 
Note that (3.20) is the partition function for the supersymmetric quantum mechanics of a spinning particle in an electro-magnetic background (with source terms), although the supersymmetry is broken by the boundary condition for the NS-NS sector. What we have done here is to rewrite the trace over the Chan-Paton Hilbert space $\mathcal{H}$ using the path integral formulation of the boundary quantum mechanics. This is the essence for reproducing the Dp-brane boundary state from that described as a bound state of infinitely many non-BPS D-instantons. We can also see that the solution part (3.7), (3.8) reproduces the base space $|B p ; \pm\rangle$, while the fluctuation part in (3.10) contributes only to the boundary interaction $e^{-S_{b}}$ for the $\mathrm{D} p$-brane.

\subsection{The scalar fields}

The massless scalar fields on the $\mathrm{D} p$-brane represent the position of the $\mathrm{D} p$-brane worldvolume in the transverse direction. The $\mathrm{D} p$-brane is now constructed by non-BPS Dinstantons whose position is represented by eigen values of the operators $\Phi^{\mu}$. Therefore, the fluctuations corresponding to the scalar fields on the $\mathrm{D} p$-brane is obtained by considering the fluctuation

$$
\Phi^{i}=\phi^{i}(\widehat{x}), \quad(i=p+1, \ldots, 9)
$$

around the solution $\Phi^{i}=0$ in (3.8). Here we abbreviated $\phi^{i}(\widehat{x}) \otimes 1$ on $\left(\mathcal{H} \otimes \mathbf{C}^{N}\right) \otimes S$ as $\phi^{i}(\widehat{x})$. Then, (3.3) becomes

$$
\begin{aligned}
M=- & \left(i \widehat{x}^{\alpha} P_{\alpha}+i \phi^{i} P_{i}+\frac{1}{2}\left[\phi^{i}, \phi^{j}\right] \Pi_{i} \Pi_{j}+u^{2}\left(\widehat{p}_{\alpha}-i A_{\alpha}\right)^{2}\right) I \\
& -i u\left(\Pi_{\alpha}+D_{\alpha} \phi^{i} \Pi_{i}\right) \Gamma^{\alpha}+\frac{u^{2}}{2} F_{\alpha \beta} \Gamma^{\alpha \beta},
\end{aligned}
$$

Repeating the analysis in the previous subsection, we obtain

$$
e^{-S_{b}\left(\Phi^{\mu}, T\right)}|B(-1) ; \pm\rangle \rightarrow e^{-S_{b}\left(A_{\alpha}, \phi^{i}\right)}|B p ; \pm\rangle
$$

in the $u \rightarrow \infty$ limit. Here

$$
e^{-S_{b}\left(A_{\alpha}, \Phi^{i}\right)}=\operatorname{tr} \mathrm{P} \exp \left\{\int d \sigma(\mathcal{A}+\mathcal{F})\right\},
$$

where $\mathcal{A}$ and $\mathcal{F}$ are defined in (2.27) and (2.29). This reproduces the correct $\phi^{i}(x)$ dependence in the boundary interaction for $\mathrm{D} p$-brane reviewed in section 2. 


\subsection{The tachyon field}

When $p$ is odd, the solution $(3.7,3.8)$ represents $N$ non-BPS D $p$-branes in type IIA string theory. And there should be a fluctuation corresponding to the tachyon field on the non-BPS D $p$-brane. Note that when $p$ is odd, there is a chirality operator $\gamma_{*}$ which anti-commutes with $\gamma_{\alpha}(\alpha=0,1, \ldots, p)$ and normalized as $\gamma_{*}^{2}=1$,

$$
\begin{aligned}
\left\{\gamma^{\alpha}, \gamma_{*}\right\} & =0, \quad(\alpha=0,1, \ldots, p) \\
\gamma_{*}^{2} & =1
\end{aligned}
$$

We can add a fluctuation proportional to $\gamma_{*}$ in (3.10) as

$$
T=u \sum_{\alpha=0}^{p}\left(\widehat{p}_{\alpha}-i A_{\alpha}(\widehat{x})\right) \otimes \gamma^{\alpha}+t(\widehat{x}) \otimes \gamma_{*} .
$$

which implies

$$
T^{2}=u^{2}\left(\widehat{p}_{\alpha}-i A_{\alpha}\right)^{2}-i u D_{\alpha} t \otimes \gamma^{\alpha} \gamma_{*}+t^{2}-\frac{u^{2}}{2} F_{\alpha \beta} \gamma^{\alpha \beta}
$$

Then, (3.28) is modified as

$$
\begin{aligned}
M= & -\left(i \widehat{x}^{\alpha} P_{\alpha}+i \phi^{i} P_{i}+\frac{1}{2}\left[\phi^{i}, \phi^{j}\right] \Pi_{i} \Pi_{j}+u^{2}\left(\widehat{p}_{\alpha}-i A_{\alpha}\right)^{2}+t^{2}\right) I \\
& -\left[\phi^{i}, t\right] \Pi_{i} \Gamma_{*}+i u D_{\alpha} t \Gamma^{\alpha} \Gamma_{*}-i u\left(\Pi_{\alpha}+D_{\alpha} \phi^{i} \Pi_{i}\right) \Gamma^{\alpha}+\frac{u^{2}}{2} F_{\alpha \beta} \Gamma^{\alpha \beta},
\end{aligned}
$$

where $\Gamma_{*}=\left(\begin{array}{c}\gamma_{*} \\ \gamma_{*}\end{array}\right)$.

It is straightforward to repeat the argument in section 3.2 and we obtain

$$
e^{-S_{b}\left(\Phi^{\mu}, T\right)}|B(-1) ; \pm\rangle \rightarrow e^{-S_{b}\left(A_{\alpha}, \phi^{i}, t\right)}|B p ; \pm\rangle
$$

in the $u \rightarrow \infty$ limit. Here the boundary interaction is given by

$$
e^{-S_{b}\left(A_{\alpha}, \Phi^{i}, T\right)}=\int d \eta \operatorname{tr} \mathrm{P} \exp \left\{\int d \sigma\left(\frac{1}{4} \dot{\eta} \eta+\mathcal{A}+\mathcal{F}-T^{2}+i \mathcal{D} T \eta\right)\right\},
$$

where $\mathcal{A}$ and $\mathcal{F}$ are those defined in (2.27) and (2.29), respectively, and $\mathcal{D} T$ is given by

$$
\mathcal{D} T=D_{\alpha} T \Psi^{\alpha}+i\left[\Phi^{i}, T\right] \Pi_{i}
$$

which is obtained by setting $\widetilde{A}_{\alpha}=A_{\alpha}$ and $\widetilde{\Phi}^{i}=\Phi^{i}$ in (2.31). This boundary interaction (3.37) is exactly what we expect for non-BPS D $p$-branes as explained in section 2. 
When $p$ is even, we can similarly construct $\mathrm{D} p-\overline{\mathrm{D} p}$ system by replacing $\gamma^{\alpha}$ with $\sigma_{3} \otimes \gamma^{\alpha}$ in (3.7). Then, $\sigma_{1} \otimes 1$ and $\sigma_{2} \otimes 1$ play the same role as $\gamma_{*}$ above. In this case, (3.27) and (3.33) are replaced as

$$
\begin{aligned}
\Phi^{i} & =\left(\begin{array}{cc}
\phi^{i}(\widehat{x}) & \\
\tilde{\phi}^{i}(\widehat{x})
\end{array}\right), \\
T & =\left(\begin{array}{cc}
u\left(\widehat{p}_{\alpha}-i A_{\alpha}(\widehat{x})\right) \otimes \gamma^{\alpha} & t(\widehat{x}) \\
t(\widehat{x})^{\dagger} & -u\left(\widehat{p}_{\alpha}-i \widetilde{A}_{\alpha}(\widehat{x})\right) \otimes \gamma^{\alpha}
\end{array}\right),
\end{aligned}
$$

and the same argument as above precisely implies the boundary state for $\mathrm{D} p-\overline{\mathrm{D} p}$ system with boundary interaction given in $(2.22)-(2.31)$.

\subsection{Superfield formulation and general fluctuations}

In section 3.2, 3.3 and 3.4, we have shown that the boundary state with the boundary interaction representing $\mathrm{D} p$-brane with the fields on it is correctly reproduced from the non-BPS D-instanton system by considering the fluctuations $A_{\alpha}, \phi^{i}$ and $t$ of the form

$$
\begin{aligned}
T & =u \sum_{\alpha=0}^{p}\left(\widehat{p}_{\alpha}-i A_{\alpha}(\widehat{x})\right) \otimes \gamma^{\alpha}+t(\widehat{x}) \otimes \gamma_{*}, \\
\Phi^{\alpha} & =\widehat{x}^{\alpha} \otimes 1 \quad(\alpha=0, \ldots, p), \quad \Phi^{i}=\phi^{i}(\widehat{x}) \otimes 1 \quad(i=p+1, \ldots, 9) .
\end{aligned}
$$

around the D-brane solution $(3.7,3.8)$. Here $A_{\alpha}, \phi^{i}$ and $t$ correspond to the gauge, scalar and tachyon fields on the $\mathrm{D} p$-brane, where the tachyon exists for the cases with odd $p$. One might think it a little ad hoc, since we have chosen the fluctuation by hand and shown that they correspond to the fields on the $\mathrm{D} p$-brane, one by one. In principle, we could consider any fluctuations around the D-brane solution and there is no reason to restrict them as above in the first place. In this subsection, we consider more general fluctuations and show that the fluctuations other than those considered above are essentially irrelevant in the limit $u \rightarrow \infty$.

Before considering fluctuations around the Dp-brane solution, we develop an efficient way to analyze it using superfield. In (2.21), we introduced a superfield description of the boundary interaction. $\boldsymbol{\Gamma}^{I}(\sigma)=\eta^{I}(\sigma)+\theta F^{I}(\sigma)$ are the fermionic superfields which correspond to gamma matrices $\Gamma^{I}$. In fact, the kinetic term for $\Gamma^{I}$ implies that $\eta^{I}$ satisfy

$$
\left\{\widehat{\eta}^{I}, \widehat{\eta}^{J}\right\}=2 \delta^{I J}
$$


in the operator formulation, which is the same anti-commutation relation as the gamma matrices. This is why we expanded the matrix $\mathbf{M}$ by the gamma matrices in (2.20). Now, let us suppose that $\mathbf{M}^{I_{1} \ldots I_{k}}$ in $(2.20)$ are operators acting on a Hilbert space $\mathcal{H}$ and can be expressed as (Weyl ordered) polynomials of the operators $\widehat{x}^{\alpha}$ and $\widehat{p}_{\alpha}$, which satisfy the canonical commutation relation (3.6). Then, following the analogous argument, we can rewrite the boundary interaction (2.21) in the path integral formulation introducing superfields $\mathbf{x}^{\alpha}(\sigma)=x^{\alpha}(\sigma)+i \theta \psi^{\alpha}(\sigma)$ and $\mathbf{p}_{\alpha}(\sigma)=p_{\alpha}(\sigma)+i \theta \pi_{\alpha}(\sigma)$, which correspond to the operators $\widehat{x}^{\alpha}$ and $\widehat{p}_{\alpha}$, respectively;

$$
\begin{aligned}
e^{-S_{b}}=\int[ & \left.d \boldsymbol{\Gamma}^{I}\right]\left[d \mathbf{x}^{\alpha}\right]\left[d \mathbf{p}_{\alpha}\right] \exp \left\{\int d \widehat { \sigma } \left(\frac{1}{4} \boldsymbol{\Gamma}^{I} D \boldsymbol{\Gamma}^{I}+i \mathbf{p}_{\alpha} D \mathbf{x}^{\alpha}\right.\right. \\
& \left.\left.+\sum_{k=0}^{2 m} \mathbf{M}^{I_{1} \cdots I_{k}}(\mathbf{x}, \mathbf{p}) \boldsymbol{\Gamma}^{I_{1}} \cdots \boldsymbol{\Gamma}^{I_{k}}\right)\right\}
\end{aligned}
$$

We will give a formal argument to show the equivalence between (3.44) and (2.38) in Appendix A.

Then, let us again consider the non-BPS D-instantons in type IIA string theory, in which the boundary interaction is given by (3.44) with

$$
\mathbf{M}=\left(\begin{array}{cc}
-i \Phi^{\mu} \mathbf{P}_{\mu} & T \\
T & -i \Phi^{\mu} \mathbf{P}_{\mu}
\end{array}\right)
$$

To see how this prescription works, we first reexamine the configuration $(3.41,3.42)$. Inserting $(3.41,3.42)$ into $(3.45)$, we obtain

$$
\mathbf{M}(\widehat{x}, \widehat{p})=-i \widehat{x}^{\alpha} \mathbf{P}_{\alpha}-i \phi^{i}(\widehat{x}) \mathbf{P}_{i}+u\left(\widehat{p}_{\alpha}-i A_{\alpha}(\widehat{x})\right) \Gamma^{\alpha}+t(\widehat{x}) \Gamma_{*} .
$$

The boundary interaction (3.44) is now

$$
\begin{aligned}
e^{-S_{b}\left(\Phi^{\mu}, T\right)}=\int[ & \left.d \boldsymbol{\Gamma}^{I}\right]\left[d \mathbf{x}^{\alpha}\right]\left[d \mathbf{p}_{\alpha}\right] \operatorname{tr} \widehat{\mathrm{P}} \exp \left\{\int d \widehat { \sigma } \left(\frac{1}{4} \boldsymbol{\Gamma}^{I} D \boldsymbol{\Gamma}^{I}+i \mathbf{p}_{\alpha} D \mathbf{x}^{\alpha}\right.\right. \\
& \left.\left.-i \mathbf{x}^{\alpha} \mathbf{P}_{\alpha}-i \phi^{i}(\mathbf{x}) \mathbf{P}_{i}+u\left(\mathbf{p}_{\alpha}-i A_{\alpha}(\mathbf{x})\right) \boldsymbol{\Gamma}^{\alpha}+t(\mathbf{x}) \boldsymbol{\Gamma}_{*}\right)\right\},
\end{aligned}
$$

where $\boldsymbol{\Gamma}^{I}=\left(\boldsymbol{\Gamma}^{\alpha}, \boldsymbol{\Gamma}_{*}\right)$. We can perform the path integral over $\mathbf{p}_{\alpha}$ which implies

$$
i D \mathbf{x}^{\alpha}+u \Gamma^{\alpha}=0
$$

and integrating out $\Gamma^{\alpha}$, we obtain

$$
\begin{aligned}
e^{-S_{b}\left(\Phi^{\mu}, T\right)=\int[} & \left.d \boldsymbol{\Gamma}_{*}\right]\left[d \mathbf{x}^{\alpha}\right] \operatorname{tr} \widehat{\mathrm{P}} \exp \left\{\int d \widehat { \sigma } \left(-\frac{1}{4 u^{2}} D \mathbf{x}^{\alpha} D^{2} \mathbf{x}^{\alpha}+\frac{1}{4} \boldsymbol{\Gamma}_{*} D \boldsymbol{\Gamma}_{*}\right.\right. \\
& \left.\left.-i \mathbf{x}^{\alpha} \mathbf{P}_{\alpha}-i \phi^{i}(\mathbf{x}) \mathbf{P}_{i}-A_{\alpha}(\mathbf{x}) D \mathbf{x}^{\alpha}+t(\mathbf{x}) \boldsymbol{\Gamma}_{*}\right)\right\} .
\end{aligned}
$$


The first term $\frac{1}{4 u^{2}} D \mathbf{x}^{\alpha} D^{2} \mathbf{x}^{\alpha}$ in the integrand of (3.49) drops in the limit $u \rightarrow \infty$ and we have

$$
e^{-S_{b}\left(\Phi^{\mu}, T\right)}|B(-1) ; \pm\rangle \rightarrow \int\left[d \boldsymbol{\Gamma}_{*}\right] \operatorname{tr} \widehat{\mathrm{P}} \exp \left\{\int d \widehat{\sigma} \mathbf{M}\left(A_{\alpha}, \phi^{i}, t\right)\right\}|B p ; \pm\rangle
$$

where $\mathbf{M}\left(A_{\alpha}, \phi^{i}, t\right)$ is

$$
\mathbf{M}\left(A_{\alpha}, \phi^{i}, t\right)=\frac{1}{4} \boldsymbol{\Gamma}_{*} D \boldsymbol{\Gamma}_{*}-i \phi^{i}(\mathbf{x}) \mathbf{P}_{i}-A_{\alpha}(\mathbf{x}) D \mathbf{x}^{\alpha}+t(\mathbf{x}) \boldsymbol{\Gamma}_{*} .
$$

The boundary interaction in the right hand side of (3.50) is nothing but that for the $\mathrm{D} p$-branes (non-BPS or BPS for odd or even $p$, respectively), which is given by (2.18) with the constraints $A_{\alpha}=\widetilde{A}_{\alpha}, \Phi^{i}=\widetilde{\Phi}^{i}$ and $T^{\dagger}=T$, as expected. Therefore, we have reproduced the same result as (3.36). However, the proof using superfields becomes much simpler than that given in the previous subsections.

Next, let us consider general fluctuations which do not involve the operator $\widehat{p}_{\alpha}$. Possible fluctuations can be written as

$$
\begin{aligned}
T & =u \widehat{p}_{\alpha} \otimes \gamma^{\alpha}+\sum_{k: \text { odd }} t_{\alpha_{1}, \ldots, \alpha_{k}}(\widehat{x}) \otimes \gamma^{\alpha_{1}, \ldots, \alpha_{k}}+\sum_{k: \text { even }} t_{* \alpha_{1}, \ldots, \alpha_{k}}(\widehat{x}) \otimes \gamma_{*} \gamma^{\alpha_{1}, \ldots, \alpha_{k}}, \\
\Phi^{\alpha} & =\widehat{x}^{\alpha} \otimes 1+\sum_{k: \text { even }} \phi_{\alpha_{1}, \ldots, \alpha_{k}}^{\alpha}(\widehat{x}) \otimes \gamma^{\alpha_{1}, \ldots, \alpha_{k}}+\sum_{k: \text { odd }} \phi_{* \alpha_{1}, \ldots, \alpha_{k}}^{\alpha}(\widehat{x}) \otimes \gamma_{*} \gamma^{\alpha_{1}, \ldots, \alpha_{k}},(3.5 \\
\Phi^{i} & =\sum_{k: \text { even }} \phi_{\alpha_{1}, \ldots, \alpha_{k}}^{i}(\widehat{x}) \otimes \gamma^{\alpha_{1}, \ldots, \alpha_{k}}+\sum_{k: \text { odd }} \phi_{* \alpha_{1}, \ldots, \alpha_{k}}^{i}(\widehat{x}) \otimes \gamma_{*} \gamma^{\alpha_{1}, \ldots, \alpha_{k}} .
\end{aligned}
$$

Here $\gamma_{*} \propto \gamma^{0} \gamma^{1} \cdots \gamma^{p}$ is a product of even number of $\gamma^{\alpha}$ for odd $p$. When $p$ is even, since we have $\gamma^{0} \gamma^{1} \cdots \gamma^{p} \propto 1$, we can simply drop the terms which involve $\gamma_{*}$ in these equations.

Then the matrix (3.45) becomes

$$
\begin{aligned}
\mathbf{M}(\widehat{x}, \widehat{p})= & -i \widehat{x}^{\alpha} \mathbf{P}_{\alpha}+u \widehat{p}_{\alpha} \Gamma^{\alpha} \\
& +\sum_{k: \text { odd }} t_{\alpha_{1}, \ldots, \alpha_{k}}(\widehat{x}) \Gamma^{\alpha_{1}, \ldots, \alpha_{k}}+\sum_{k: \text { even }} t_{* \alpha_{1}, \ldots, \alpha_{k}}(\widehat{x}) \Gamma_{*} \Gamma^{\alpha_{1}, \ldots, \alpha_{k}} \\
& -i \sum_{k: \text { even }} \phi_{\alpha_{1}, \ldots, \alpha_{k}}^{\mu}(\widehat{x}) \Gamma^{\alpha_{1}, \ldots, \alpha_{k}} \mathbf{P}_{\mu}-i \sum_{k: \text { odd }} \phi_{* \alpha_{1}, \ldots, \alpha_{k}}^{\mu}(\widehat{x}) \Gamma_{*} \Gamma^{\alpha_{1}, \ldots, \alpha_{k}} \mathbf{P}_{\mu} .
\end{aligned}
$$


Performing the path integral over $\mathbf{p}_{\alpha}$, we again obtain (3.48) and, as a result, the vertex operators

$$
\begin{aligned}
& \sum_{k: \text { odd }} \frac{1}{u^{k}} t_{\alpha_{1}, \ldots, \alpha_{k}} D \mathbf{X}^{\alpha_{1}} \cdots D \mathbf{X}^{\alpha_{k}}, \sum_{k: \text { even }} \frac{1}{u^{k}} t_{* \alpha_{1}, \ldots, \alpha_{k}} D \mathbf{X}^{\alpha_{1}} \cdots D \mathbf{X}^{\alpha_{k}}, \\
& \sum_{k: \text { even }} \frac{1}{u^{k}} \phi_{\alpha_{1}, \ldots, \alpha_{k}}^{\mu} D \mathbf{X}^{\alpha_{1}} \cdots D \mathbf{X}^{\alpha_{k}} \mathbf{P}_{\mu}, \quad \sum_{k: \text { odd }} \frac{1}{u^{k}} \phi_{* \alpha_{1}, \ldots, \alpha_{k}}^{\mu} D \mathbf{X}^{\alpha_{1}} \cdots D \mathbf{X}^{\alpha_{k}} \mathbf{P}_{\mu},
\end{aligned}
$$

are turned on in the boundary interaction. In order for these fields to survive in the $u \rightarrow \infty$ limit, $t_{\alpha_{1} \ldots \alpha_{k}}, t_{* \alpha_{1} \ldots \alpha_{k}}, \phi_{\alpha_{1} \ldots \alpha_{k}}^{\mu}$ and $\phi_{* \alpha_{1} \ldots \alpha_{k}}^{\mu}$, should be of order $u^{k}$. If we allow only such fluctuations that do not increase more than the $\mathrm{D} p$-brane solution itself in the $u \rightarrow \infty$ limit, that is,

$$
\begin{aligned}
T & =u \sum_{\alpha=0}^{p} \widehat{p}_{\alpha} \otimes \gamma^{\alpha}+\mathcal{O}\left(u^{1}\right), \\
\Phi^{\alpha} & =\widehat{x}^{\alpha} \otimes 1+\mathcal{O}\left(u^{0}\right), \quad(\alpha=0, \ldots, p), \\
\Phi^{i} & =\mathcal{O}\left(u^{0}\right), \quad(i=p+1, \ldots, 9),
\end{aligned}
$$

the only relevant fluctuations are

$$
\begin{aligned}
T & =u \sum_{\alpha=0}^{p}\left(\widehat{p}_{\alpha}-i A_{\alpha}(\widehat{x})\right) \otimes \gamma^{\alpha}+t_{*}(\widehat{x}) \otimes \gamma_{*}, \\
\Phi^{\alpha} & =\widehat{x}^{\alpha} \otimes 1+\phi^{\alpha}(\widehat{x}) \otimes 1, \quad(\alpha=0, \ldots, p), \\
\Phi^{i} & =\phi^{i}(\widehat{x}) \otimes 1, \quad(i=p+1, \ldots, 9),
\end{aligned}
$$

where we have set $t_{\alpha}=-i u A_{\alpha}$.

The fluctuation $\phi^{\alpha}$ in (3.61) represent the non-Abelian generalization of the reparametrization of the world-volume coordinates. When the fluctuations are Abelian, $\phi^{\alpha}$ can be absorbed by the gauge transformation [11]. In fact, the gauge transformation (3.9) with $U=\exp \left(i\left\{\widehat{p}_{\alpha}, \lambda^{\alpha}(\widehat{x})\right\} / 2\right)$ implies

$$
U \widehat{x}^{\alpha} U^{-1}=\widehat{x}^{\alpha}+\lambda^{\alpha}(\widehat{x})+\mathcal{O}\left(\lambda^{2}\right)
$$

and we can always set $\phi^{\alpha}=0$ by choosing $\lambda^{\alpha}$ appropriately. This comes from the reparametrization invariance of the $\mathrm{D} p$-brane world-volume, which is included in the 
gauge symmetry in the K-matrix theory [11]. For the non-Abelian cases, we cannot always set $\phi^{\alpha}=0$. For example, if a commutator $\left[\phi^{\alpha}, \phi^{\beta}\right]$ is non-zero, there is no way to set this quantity to zero by unitary transformation. We can try the non-Abelian generalization of the gauge transformation (3.63) to absorb the fluctuations, but in this case, the $\mathcal{O}\left(\lambda^{2}\right)$ terms involve $\widehat{p}_{\alpha}$ and the $\widehat{p}_{\alpha}$ dependent fluctuations are induced. Note also that even in the Abelian cases, the transformation (3.63) will induce $\widehat{p}_{\alpha}$ dependent fluctuations in the tachyon operator (3.60), which corresponds to the transformation of vielbein.

So far we have restricted the fluctuations to be independent of the operator $\widehat{p}_{\alpha}$. It is also important to see what happens if we include the $\widehat{p}_{\alpha}$ dependent fluctuations. We can apply the above prescription at least when the fluctuations are linear with respect to $\widehat{p}_{\alpha}$. Let us add such fluctuations to (3.60)-(3.62) and consider

$$
\begin{aligned}
T & =u e_{\beta}^{\alpha}(\widehat{x}) \widehat{D}_{\alpha} \otimes \gamma^{\beta}+\left(t_{*}(\widehat{x})+t_{*}^{\alpha}(\widehat{x}) \widehat{D}_{\alpha}\right) \otimes \gamma_{*} \\
\Phi^{\alpha} & =\left(\widehat{x}^{\alpha}+\phi^{\alpha}(\widehat{x})+\phi^{\alpha \beta}(\widehat{x}) \widehat{D}_{\beta}\right) \otimes 1, \quad(\alpha=0, \ldots, p), \\
\Phi^{i} & =\left(\phi^{i}(\widehat{x})+\phi^{i \alpha}(\widehat{x}) \widehat{D}_{\alpha}\right) \otimes 1, \quad(i=p+1, \ldots, 9),
\end{aligned}
$$

where $\widehat{D}_{\alpha}=\widehat{p}_{\alpha}-i A_{\alpha}(\widehat{x})$ and the products of $\widehat{x}^{\alpha}$ and $\widehat{p}_{\alpha}$ are understood as Weyl ordered products. We have ignored higher terms in the gamma matrix expansion since they do not contribute in the limit $u \rightarrow \infty$ from the argument given above. Here we consider the Abelian case for simplicity. The boundary interaction is again given by inserting this configuration into (3.44) and (3.45);

$$
\begin{aligned}
e^{-S_{b}}=\int[ & \left.d \boldsymbol{\Gamma}^{I}\right]\left[d \mathbf{x}^{\alpha}\right]\left[d \mathbf{p}_{\alpha}\right] \exp \left\{\int d \widehat{\sigma}\left(\frac{1}{4} \boldsymbol{\Gamma}^{I} D \boldsymbol{\Gamma}^{I}+i \mathbf{p}_{\alpha} D \mathbf{x}^{\alpha}+\mathbf{M}(\mathbf{x}, \mathbf{p})\right)\right\} \\
\mathbf{M}(\mathbf{x}, \mathbf{p})= & -i\left(\mathbf{x}^{\alpha}+\phi^{\alpha}(\mathbf{x})+\phi^{\alpha \beta}(\mathbf{x})\left(\mathbf{p}_{\beta}-i A_{\beta}(\mathbf{x})\right)\right) \mathbf{P}_{\alpha} \\
& -i\left(\phi^{i}(\mathbf{x})+\phi^{i \alpha}(\mathbf{x})\left(\mathbf{p}_{\alpha}-i A_{\alpha}(\mathbf{x})\right)\right) \mathbf{P}_{i} \\
& +u e_{\beta}^{\alpha}(\mathbf{x})\left(\mathbf{p}_{\alpha}-i A_{\alpha}(\mathbf{x})\right) \boldsymbol{\Gamma}^{\beta}+\left(t_{*}(\mathbf{x})+t_{*}^{\alpha}(\mathbf{x})\left(\mathbf{p}_{\alpha}-i A_{\alpha}(\mathbf{x})\right) \boldsymbol{\Gamma}_{*} .\right.
\end{aligned}
$$

Performing the path integral with respect to $\mathbf{p}_{\alpha}$, we obtain a delta-functional which 
imposes the condition 13

$$
i D \mathbf{x}^{\alpha}+u e_{\beta}^{\alpha}(\mathbf{x}) \boldsymbol{\Gamma}^{\beta}+t_{*}^{\alpha}(\mathbf{x}) \boldsymbol{\Gamma}_{*}-i \phi^{\beta \alpha}(\mathbf{x}) \mathbf{P}_{\beta}-i \phi^{i \alpha}(\mathbf{x}) \mathbf{P}_{i}=0
$$

Then, integrating out $\boldsymbol{\Gamma}^{\beta}$, we can easily see that all the new degrees of freedom (i.e. $e_{\beta}^{\alpha}, t_{*}^{\alpha}, \phi^{\alpha \beta}$ and $\left.\phi^{i \alpha}\right)$ will be dropped in the limit $u \rightarrow \infty$. Therefore, there is no effect from the fluctuations which depend linearly on $\widehat{p}_{\alpha}$. We do not proceed to a systematic analysis including higher order terms with respect to $\widehat{p}_{\alpha}$ for a technical reason. But, we expect that there are essentially no new degrees of freedom. Roughly speaking, even if there are such higher order terms, the largest contribution in the boundary interaction (3.67) will be $u e_{\beta}^{\alpha}(\mathbf{x})\left(\mathbf{p}_{\alpha}-i A_{\alpha}(\mathbf{x})\right) \boldsymbol{\Gamma}^{\beta}$ in the $u \rightarrow \infty$ limit. This will imply the condition $\mathbf{p}_{\alpha}-i A_{\alpha}(\mathbf{x})=0$, from which we can eliminate all the $\widehat{p}_{\alpha}$ dependent fluctuations.

\subsection{CS-term and the index theorem}

Here we consider the Chern-Simons term. Since we have already shown that the $\mathrm{D} p$ brane boundary state can be obtained by considering fluctuations around the $\mathrm{D} p$-brane solution in K-matrix theory, it is now clear that the CS-term of D $p$-brane can be obtained from that of K-matrix theory. ${ }^{14}$ More explicitly, (2.41) and (3.36) imply

$$
S_{C S}^{D(-1)}\left(C, \Phi^{\mu}, T\right)=S_{C S}^{D p}\left(C, A_{\mu}, \phi^{i}, t\right)
$$

inserting the configuration (3.41) and (3.42) in the limit $u \rightarrow \infty$. As we can see in (3.70), the left hand side is written by operators $\Phi^{\mu}$ and $T$ acting on a Hilbert space, while the right hand side is written in terms of the fields $A_{\mu}, \phi^{i}$ and $t$ on the $\mathrm{D} p$-brane. For example, if we consider BPS D $p$-branes, in which $t$ doesn't exist, and turn off the scalar fields $\phi^{i}$, the CS-term for the $\mathrm{D} p$-brane takes the usual form

$$
S_{C S}^{D p}\left(C, A_{\mu}\right)=\mu_{p} \int_{D p} C \wedge \operatorname{tr} e^{2 \pi F}
$$

\footnotetext{
${ }^{13}$ To be more precise, we should understand the operators $\mathbf{P}_{\beta}$ and $\mathbf{P}_{i}$ as their eigen functions by using (4.4).

${ }^{14}$ We can also obtain, for example, DBI action from K-matrix theory as done in [9] explicitly for transverse scalar fields of the $\mathrm{D} p$-brane although we do not discuss it in this paper.
} 
up to derivative corrections which do not contribute to the D-brane charges. Comparing the coupling to the RR-fields $C$ in the both sides of (3.70), we obtain some interesting formulae which relate some quantity written in the operator theory with those written in the gauge theory. ${ }^{15}$ In particular, as we will see below, this observation naturally leads us to obtain a physical derivation of Atiyah-Singer index theorem.

In the following, we will first derive general CS-terms in our formulation. It would be instructive to calculate the CS-term and show the equivalence (3.70) explicitly. Then, we will give the physical interpretation of Atiyah-Singer index theorem as well as its generalization.

\subsubsection{General CS-terms}

As we briefly mentioned in (2.41), the CS-term for the $\mathrm{D} p$-brane can be written as

$$
S_{C S}^{D p}=\left\langle C\left|\operatorname{Str} \mathrm{P} e^{\int d \sigma M}\right| B p ;+\right\rangle_{\mathrm{RR}}
$$

using the boundary interaction of the form (2.34). Here we concentrate on the lowest order term in the derivative expansion. Then, it is known that only zero modes contribute in the calculation because of the supersymmetry (See, for example, [28, 27]). Let $\psi_{1}^{\mu}$ and $i \psi_{2}^{\mu} / 2 \pi$ be the zero modes of $\Psi^{\mu}$ and $\Pi^{\mu}$, respectively, satisfying

$$
\left\{\psi_{1}^{\mu}, \psi_{1}^{\nu}\right\}=\left\{\psi_{2}^{\mu}, \psi_{2}^{\nu}\right\}=0, \quad\left\{\psi_{1}^{\mu}, \psi_{2}^{\nu}\right\}=\delta^{\mu \nu}
$$

Then, inserting the zero mode part of the expressions (2.27)-(2.31) and the boundary state (2.1) into (3.72), we obtain

$$
S_{C S}^{D p}=\mu_{p} \int \prod_{\beta=0}^{p} d x^{\beta} d \psi_{1}^{\beta}\left\langle\psi_{2}^{i}=0\left|\int d^{9-p} k C\left(x^{\alpha}, k_{i}, \psi_{1}^{\mu}\right) J\left(x^{\alpha}, k_{i}, \psi_{1}^{\alpha}, \psi_{2}^{i}\right)\right| \psi_{1}^{i}=0\right\rangle,
$$

where

$$
\begin{aligned}
C\left(x^{\mu}, \psi_{1}^{\mu}\right) & =\sum_{n} C_{\mu_{1} \cdots \mu_{n}}\left(x^{\mu}\right) \psi_{1}^{\mu_{1}} \cdots \psi_{1}^{\mu_{n}} \\
& =\int d^{9-p} k e^{i x^{j} k_{j}} C\left(x^{\alpha}, k_{i}, \psi_{1}^{\mu}\right)
\end{aligned}
$$

\footnotetext{
${ }^{15}$ Similar strategy was used in the context of non-commutative gauge theory in [30]-[32], in which Seiberg-Witten map is derived by comparing the RR-coupling in commutative and non-commutative description of a gauge theory.
} 
and

$$
\begin{gathered}
J\left(x^{\alpha}, k_{i}, \psi_{1}^{\alpha}, \psi_{2}^{i}\right)=\operatorname{Str}\left(e^{-i k_{i} \widehat{\Phi}^{i}+2 \pi \widehat{\mathcal{F}}}\right) \\
\widehat{\Phi}^{i}=\left(\begin{array}{cc}
\Phi^{i} & \\
\widetilde{\Phi}^{i}
\end{array}\right), \quad \widehat{\mathcal{F}}=\left(\begin{array}{cc}
-T T^{\dagger}+\mathcal{F} & \mathcal{D} T \\
\mathcal{D} T^{\dagger} & -T^{\dagger} T+\widetilde{\mathcal{F}}
\end{array}\right), \\
\mathcal{F}=\frac{1}{2} F_{\alpha \beta} \psi_{1}^{\alpha} \psi_{1}^{\beta}-\frac{1}{2 \pi}\left(\partial_{\alpha} \Phi^{i}+\left[A_{\alpha}, \Phi^{i}\right]\right) \psi_{1}^{\alpha} \psi_{2}^{i}+\frac{1}{8 \pi^{2}}\left[\Phi^{i}, \Phi^{j}\right] \psi_{2}^{i} \psi_{2}^{j}, \\
\widetilde{\mathcal{F}}=\frac{1}{2} \widetilde{F}_{\alpha \beta} \psi_{1}^{\alpha} \psi_{1}^{\beta}-\frac{1}{2 \pi}\left(\partial_{\alpha} \widetilde{\Phi}^{i}+\left[\widetilde{A}_{\alpha}, \widetilde{\Phi}^{i}\right]\right) \psi_{1}^{\alpha} \psi_{2}^{i}+\frac{1}{8 \pi^{2}}\left[\widetilde{\Phi}^{i}, \widetilde{\Phi}^{j}\right] \psi_{2}^{i} \psi_{2}^{j}, \\
\mathcal{D} T^{\dagger}=i\left(\partial_{\alpha} T+A_{\alpha} T-T \widetilde{A}_{\alpha}\right) \psi_{1}^{\alpha}-\frac{i}{2 \pi}\left(\Phi^{i} T-T \widetilde{\Phi}^{i}\right) \psi_{2}^{i} \\
i\left(\partial_{\alpha} T^{\dagger}+\widetilde{A}_{\alpha} T^{\dagger}-T^{\dagger} A_{\alpha}\right) \psi_{1}^{\alpha}-\frac{i}{2 \pi}\left(\widetilde{\Phi}^{i} T^{\dagger}-T^{\dagger} \Phi^{i}\right) \psi_{2}^{i} .
\end{gathered}
$$

Here the indices run as $\mu, \nu=0, \ldots, 9, \alpha, \beta=0, \ldots, p$ and $i, j=p+1, \ldots, 9$. In (3.74), the zero modes of the operators $X^{\alpha}, P_{i}$ and $\Psi^{\alpha}$ in the boundary interaction are replaced with their eigen values denoted as $x^{\alpha}, k_{i}$ and $\psi_{1}^{\alpha}$, respectively, while we still treat $\psi_{1}^{i}$ and $\psi_{2}^{i}$ as fermionic operators satisfying (3.73). $\left\langle\psi_{2}^{i}=0\right|$ and $\left|\psi_{1}^{i}=0\right\rangle$ are bra and ket states which are annihilated by $\psi_{2}^{i}$ and $\psi_{1}^{i}$, respectively, satisfying $\left\langle\psi_{2}^{i}=0 \mid \psi_{1}^{i}=0\right\rangle=1 .\left\langle\psi_{2}^{i}=0\right|$ is originally a zero momentum Fock vacuum on which RR-states are constructed, ${ }^{16}$ while $\left|\psi_{1}^{i}=0\right\rangle$ is the zero mode part of the coherent state $\left|x^{\alpha}, x^{i}=0\right\rangle\left|\psi^{\alpha}, \psi^{i}=0\right\rangle_{\mathrm{RR}}$. The normalization factor $\mu_{p}=1 /(2 \pi)^{p+1}$ in $(3.74)$ comes from the path integration with respect to the non-zero modes. (See Appendix B.)

When the scalar fields $\Phi^{i}, \widetilde{\Phi}^{i}$ are turned off, this CS-term will be reduced to the well-known form $[34,20,21]$

$$
S_{C S}^{D p}=\mu_{p} \int C \wedge \operatorname{Str} e^{2 \pi \widehat{\mathcal{F}}}
$$

with the supercurvature [35]

$$
\widehat{\mathcal{F}}=\left(\begin{array}{cc}
-T T^{\dagger}+\frac{1}{2} F_{\alpha \beta} d x^{\alpha} d x^{\beta} & i D_{\alpha} T d x^{\alpha} \\
i D_{\alpha} T^{\dagger} d x^{\alpha} & -T^{\dagger} T+\frac{1}{2} \widetilde{F}_{\alpha \beta} d x^{\alpha} d x^{\beta}
\end{array}\right) .
$$

\footnotetext{
${ }^{16}$ See $[33,28]$ for more precise argument.
} 
For the BPS D $p$-branes, we can rewrite (3.74) in more familiar form. The CS-term for the BPS D $p$-brane can be obtained by replacing (3.77) with

$J\left(x^{\alpha}, k_{i}, \psi_{1}^{\alpha}, \psi_{2}^{i}\right)=\operatorname{Tr}\left(\exp \left\{-i k_{i} \Phi^{i}+\pi F_{\alpha \beta} \psi_{1}^{\alpha} \psi_{1}^{\beta}-D_{\alpha} \Phi^{i} \psi_{1}^{\alpha} \psi_{2}^{i}+\frac{1}{4 \pi}\left[\Phi^{i}, \Phi^{j}\right] \psi_{2}^{i} \psi_{2}^{j}\right\}\right)$.

We use the notation

$$
C\left(y^{\mu}, \psi_{1}^{\mu}\right) \equiv \int d^{9-p} k C\left(x^{\alpha}, k_{i}, \psi_{1}^{\mu}\right) e^{-i k_{i} \Phi^{i}},
$$

where $y^{\mu}=\left(x^{\alpha}, \Phi^{i}\left(x^{\alpha}\right)\right)$, in the following. This is just the usual Fourier transformation in the case with a single $\mathrm{D} p$-brane.

As a warm up exercise, let us first explain the case with a single $\mathrm{D} p$-brane. In this case, every field commutes with each other and we obtain

$$
\begin{aligned}
S_{C S}^{D p} & =\mu_{p} \int \prod_{\beta} d x^{\beta} d \psi_{1}^{\beta}\left\langle\psi_{2}^{i}=0\left|C\left(y^{\mu}, \psi_{1}^{\mu}\right) e^{\partial_{\alpha} y^{i} \psi_{1}^{\alpha} \psi_{2}^{i}}\right| \psi_{1}^{i}=0\right\rangle e^{\pi F_{\alpha \beta} \psi_{1}^{\alpha} \psi_{1}^{\beta}} \\
& =\mu_{p} \int \prod_{\beta} d x^{\beta} d \psi_{1}^{\beta} C\left(y^{\mu}, \partial_{\alpha} y^{\mu} \psi_{1}^{\alpha}\right) e^{\pi F_{\alpha \beta} \psi_{1}^{\alpha} \psi_{1}^{\beta}}
\end{aligned}
$$

Furthermore, note that $\psi_{1}^{\beta}$ anti-commutes with each other and the integral with respect to $\psi_{1}^{\beta}$ picks up the coefficient of $\psi_{1}^{0} \psi_{1}^{1} \cdots \psi_{1}^{p}$. This enables us to regard $\psi_{1}^{\alpha}$ as the 1 -form $d x^{\alpha}$ on the world-volume. Thus we reproduce the CS-term for the BPS D $p$-brane,

$$
S_{C S}^{D p}=\mu_{p} \int_{D p} C\left(y^{\mu}, d y^{\mu}\right) \wedge e^{2 \pi F}
$$

where $C\left(y^{\mu}, d y^{\mu}\right)$ is a pull back of the RR-field to the $\mathrm{D} p$-brane world-volume and $F=\frac{1}{2} F_{\alpha \beta} d x^{\alpha} \wedge d x^{\beta}$.

Next, we consider the non-Abelian case. Note that when we expand the exponential in (3.77), the matrices $\Phi^{i}, D_{\alpha} \Phi^{i},\left[\Phi^{i}, \Phi^{j}\right]$ and $F_{\alpha \beta}$ always appear in symmetric order. Therefore, we can forget about the ordering of these by simply replacing the trace $\operatorname{Tr}$ with the symmetrized trace. So we can proceed as in the Abelian case except the contribution of the last term in (3.85). By noticing that $\psi_{2}^{i}$ can be identified as $\frac{\partial}{\partial \psi_{1}^{i}}$, we see that this term simply modifies the RR-field $C$ to

$$
C_{\left[y^{\mu}, y^{\nu}\right]}\left(y^{\mu}, \psi_{1}^{\mu}\right) \equiv e^{\frac{1}{4 \pi}\left[y^{\mu}, y^{\nu}\right] \frac{\partial}{\partial \psi_{1}^{\mu} \wedge} \frac{\partial}{\partial \psi_{1}^{\nu}}} C\left(y^{\mu}, \psi_{1}^{\mu}\right) .
$$


This corresponds to inclusion of Myers term [36]. Then, the CS-term (3.74) will become

$$
S_{C S}^{D p}=\mu_{p} \int_{D p} \operatorname{Sym} \operatorname{Tr}\left(C_{\left[y^{\mu}, y^{\nu}\right]}\left(y^{\mu}, D y^{\mu}\right) \wedge e^{2 \pi F}\right)
$$

where $D y^{\mu}=D_{\alpha} y^{\mu}(x) d x^{\alpha}$ and SymTr denotes the symmetrized trace with respect to $y^{\mu}, D_{\alpha} y^{\mu}, F_{\alpha \beta}$ and $\left[y^{\mu}, y^{\nu}\right]$.

\subsubsection{Ascent relations of CS-terms}

Now we explicitly show the equivalence (3.70) between the CS-term for the D $p$-branes and that for the unstable D-instanton system in the background of the D $p$-brane solution, using the expressions given in the last subsection. Let us explain this in type IIA string theory. Our starting point is the CS-term for the non-BPS D-instantons [11, 21] which is obtained by considering $p=-1$ case in (3.74);

$$
S_{C S}^{D(-1)}=\left\langle\int d^{10} k C\left(k_{\mu}, \psi_{1}^{\mu}\right) J\left(k_{\mu}, \psi_{2}^{\mu}\right)\right\rangle
$$

where $\langle\cdots\rangle$ denotes $\left\langle\psi_{2}^{\mu}=0|\cdots| \psi_{1}^{\mu}=0\right\rangle$ and

$$
\begin{aligned}
J\left(k, \psi_{2}\right) & =\operatorname{Str}\left(e^{-i k_{\mu} \Phi^{\mu}+2 \pi \widehat{\mathcal{F}}}\right) \\
\widehat{\mathcal{F}} & =-T^{2}+\frac{1}{8 \pi^{2}}\left[\Phi^{\mu}, \Phi^{\nu}\right] \psi_{2}^{\mu} \psi_{2}^{\nu}-\frac{i}{2 \pi}\left[\Phi^{\mu}, T\right] \psi_{2}^{\mu} \sigma_{1} .
\end{aligned}
$$

Here the Pauli matrix $\sigma_{1}$ is considered as fermionic matrix which anti-commutes with $\psi_{1}^{\mu}$ and $\psi_{2}^{\mu}$, as explained in section 2 .

Let us consider the solution representing BPS D $p$-branes ( $p=$ even) and the fluctuations around it. Inserting (3.60)-(3.62) into (3.94) we obtain

$\widehat{\mathcal{F}}=-u^{2}\left(\widehat{p}_{\alpha}-i A_{\alpha}(\widehat{x})\right)^{2}+\frac{1}{8 \pi^{2}}\left[y^{\mu}(\widehat{x}), y^{\nu}(\widehat{x})\right] \psi_{2}^{\mu} \psi_{2}^{\nu}+\frac{u}{2 \pi} D_{\alpha} y^{\mu}(\widehat{x}) \psi_{2}^{\mu} \Gamma^{\alpha}+\frac{u^{2}}{2} F_{\alpha \beta}(\widehat{x}) \Gamma^{\alpha \beta}$,

Here we have introduced

$$
y^{\mu}(x)=\left(x^{\alpha}+\phi^{\alpha}(x), \phi^{i}(x)\right)
$$

which is a non-Abelian generalization of positions of the BPS D $p$-brane world-volume. 
Then we rewrite $J\left(k, \psi_{2}\right)$ in path-integral formulation with respect to $\hat{x}, \hat{p}$ and $\Gamma$ as in the previous subsections. Actually, we have already done the relevant calculations in section 3.2. The result is

$$
J\left(k, \psi_{2}\right)=\int\left[d x^{\alpha}\right]\left[d \psi^{\alpha}\right] \operatorname{tr} \mathrm{P} \exp \left(-\int d \sigma L\left(x^{\alpha}, \psi^{\alpha}, k_{\mu}, \psi_{2}^{\mu}\right)\right),
$$

where the Lagrangian $L\left(x, \psi, k, \psi_{2}\right)$ is given by

$$
\begin{aligned}
L\left(x, \psi, k, \psi_{2}\right)= & \frac{1}{4 u^{2}}\left(\dot{x}_{\alpha}^{2}+\psi^{\alpha} \dot{\psi}^{\alpha}\right)+\frac{i}{2 \pi} y^{\mu}(x) k_{\mu}-\frac{1}{2 \pi} D_{\alpha} y^{\mu}(x) \psi_{2}^{\mu} \psi^{\alpha} \\
& -\frac{1}{8 \pi^{2}}\left[y^{\mu}(x), y^{\nu}(x)\right] \psi_{2}^{\mu} \psi_{2}^{\nu}+A_{\alpha}(x) \dot{x}^{\alpha}-\frac{1}{2} F_{\alpha \beta}(x) \psi^{\alpha} \psi^{\beta} .
\end{aligned}
$$

The trace tr in (3.97) is taken with respect to the gauge indices of the resulting BPS D $p$-branes.

The path-integration of the non-zero modes of $x(\sigma)$ and $\psi(\sigma)$ in (3.97) is trivial by the supersymmetry [20] and we get

$$
\begin{aligned}
S_{C S}= & \mu_{p}\left\langle\int d^{10} k d^{p+1} x d^{p+1} \psi C\left(k^{\mu}, \psi_{1}^{\mu}\right) \times\right. \\
& \left.\operatorname{tr} \exp \left(-i y^{\mu} k_{\mu}+D_{\alpha} y^{\mu}(x) \psi_{2}^{\mu} \psi^{\alpha}+\pi F_{\alpha \beta}(x) \psi^{\alpha} \psi^{\beta}+\frac{1}{4 \pi}\left[y^{\mu}(x), y^{\nu}(x)\right] \psi_{2}^{\mu} \psi_{2}^{\nu}\right)\right\rangle \\
= & \mu_{p}\left\langle\int_{D p} \int d^{10} k C\left(k^{\mu}, \psi_{1}^{\mu}\right) \operatorname{tr} e^{\left(-i y^{\mu} k_{\mu}+D_{\alpha} y^{\mu} \psi_{2}^{\mu} d x^{\alpha}+\pi F_{\alpha \beta} d x^{\alpha} \wedge d x^{\beta}+\frac{1}{4 \pi}\left[y^{\mu}, y^{\nu}\right] \psi_{2}^{\mu} \psi_{2}^{\nu}\right)}\right\rangle \\
= & \mu_{p} \int_{D p} \operatorname{Sym} \operatorname{tr}\left(C_{\left[y^{\mu}, y^{\nu}\right]}\left(y^{\mu}, D y^{\mu}\right) \wedge e^{2 \pi F}\right) .
\end{aligned}
$$

This correctly reproduces the CS-term for BPS D $p$-branes (3.91) when we choose the static gauge $\phi^{\alpha}=0$.

\subsubsection{An interpretation of the index theorem}

Here we give an interpretation of the index theorem from the point of view of the Kmatrix theory. We will consider BPS Dp-branes constructed from type IIB K-matrix theory, i.e. an unstable system with infinitely many D-instanton - anti D-instanton pairs since it is directly related to the well-known Atiyah-Singer index theorem, though generalization to other cases is straightforward.

The Chern-Simons term of the D-instanton - anti D-instanton system can be written as (3.92), while (3.93) is now replaced with

$$
J\left(k, \psi_{2}\right)=\operatorname{Str}\left(e^{-i k_{\mu} \widehat{\Phi}^{\mu}+2 \pi \widehat{\mathcal{F}}}\right)
$$




$$
\widehat{\mathcal{F}}=\left(\begin{array}{cc}
-T T^{\dagger}+\frac{1}{8 \pi^{2}}\left[\Phi^{\mu}, \Phi^{\nu}\right] \psi_{2}^{\mu} \psi_{2}^{\nu} & -\frac{i}{2 \pi}\left(\Phi^{\mu} T-T \widetilde{\Phi}^{\mu}\right) \psi_{2}^{\mu} \\
-\frac{i}{2 \pi}\left(\widetilde{\Phi}^{\mu} T^{\dagger}-T^{\dagger} \Phi^{\mu}\right) \psi_{2}^{\mu} & -T^{\dagger} T+\frac{1}{8 \pi^{2}}\left[\widetilde{\Phi}^{\mu}, \widetilde{\Phi}^{\nu}\right] \psi_{2}^{\mu} \psi_{2}^{\nu}
\end{array}\right)
$$

Note that (3.101) can also be written as

$$
\widehat{\mathcal{F}}=-Z^{2}, \quad Z=\left(\begin{array}{cc}
\frac{i}{2 \pi} \Phi^{\mu} \psi_{2}^{\mu} & T \\
T^{\dagger} & \frac{i}{2 \pi} \widetilde{\Phi}^{\mu} \psi_{2}^{\mu}
\end{array}\right) .
$$

Let us assume that the RR-field is constant (i.e. $\left.k_{\mu}=0\right)$ and all the components except for the zero-form part $C_{0}$ are zero. Then the CS-term takes very simple form, since scalar fields do not contribute to it. Actually, it becomes the index of the tachyon operator,

$$
S_{C S}^{D(-1)}=C_{0} \operatorname{Str}\left(e^{-2 \pi Q^{2}}\right)=C_{0} \operatorname{Tr}\left((-1)^{F} e^{-2 \pi Q^{2}}\right)=C_{0} \operatorname{Index} Q .
$$

where $(-1)^{F}=\sigma^{3}$ and

$$
Q \equiv\left(\begin{array}{cc}
0 & T \\
T^{\dagger} & 0
\end{array}\right)
$$

Since the coupling of the RR $p$-form field represents $\mathrm{D}(p-1)$-brane charge, we conclude that the index of the tachyon operator is interpreted as the D-instanton charge [11]. This can also be seen from the fact that in the case $T$ is a Fredholm operator, $\operatorname{dim} \operatorname{Ker} T T^{\dagger}$ and $\operatorname{dim} \operatorname{Ker} T^{\dagger} T$ are finite dimensional and correspond to the number of D-instantons and anti D-instantons which are not annihilated by the tachyon condensation, respectively. It follows that the total D-instanton number is given by

$$
\operatorname{dim} \operatorname{Ker} T T^{\dagger}-\operatorname{dim} \operatorname{Ker} T^{\dagger} T=\operatorname{Index} Q
$$

which is another definition of the Index $Q$.

Let us next consider the D-instanton charge in the presence of BPS D $p$-branes. The tachyon configuration representing $\mathrm{D} p$-brane in type IIB K-matrix theory is also given as (3.10) and we have

$$
Q=u \sum_{\alpha=0}^{p}\left(\widehat{p}_{\alpha}-i A_{\alpha}(\widehat{x})\right) \Gamma^{\alpha} \equiv-i u \not D
$$

where $\Gamma^{\alpha}(\alpha=0, \ldots, p)$ are $S O(p+1)$ gamma matrices $\left(p=\right.$ odd). Note that $\sigma_{3}$ can be regarded as the chirality operator. Therefore, according to the above observation, 
the D-instanton charge in the presence of the BPS D $p$-branes is just the index of the usual Dirac operator $\not D$ on the world-volume of the $\mathrm{D} p$-branes in the presence of an electro-magnetic background.

On the other hand, as we have explicitly shown in the previous subsection, the CS-term for the D-instanton $S_{C S}^{D(-1)}$ in the background of $\mathrm{D} p$-brane is identical to the CS-term of $\mathrm{D} p$-brane $S_{C S}^{D p}$. In our case with $C=C_{0}=$ constant, the CS-term of $\mathrm{D} p$-brane $S_{C S}^{D p}$ is given as

$$
S_{C S}^{D p}=\mu_{p} \int_{D p} C \wedge \operatorname{tr} e^{2 \pi F}=C_{0} \int_{D p} \operatorname{tr} e^{F / 2 \pi},
$$

where tr means the trace for the gauge indices. Comparing this with (3.103), we obtain

$$
\operatorname{Index}(-i \not D)=\int_{D p} \operatorname{tr} e^{F / 2 \pi}
$$

This is nothing but the Atiyah-Singer index theorem [37]. It is quite interesting that we have naturally reached this result considering D-brane physics. The physical interpretation is now clear. The Dirac operator is the tachyon operator which represent the $\mathrm{D} p$-brane in the D-instanton - anti D-instanton system and its index gives the D-instanton charge. The D-instanton can also be constructed as the instanton configuration in the gauge theory on the $\mathrm{D} p$-brane world-volume and the instanton number is given by the Chern number of the gauge bundle. And the two descriptions actually agree as expected.

We can also include a nontrivial internal metric in the Dirac operator (3.106) as

$$
Q=-i u \not D=-i u \sum_{\alpha=0}^{p} e_{A}^{\alpha}(\widehat{x})\left(\widehat{\partial}_{\alpha}+\omega_{\alpha}(\widehat{x})+A_{\alpha}(\widehat{x})\right) \Gamma^{A},
$$

where $\widehat{\partial}_{\alpha}=i \widehat{p}_{\alpha}$ and $\omega_{\alpha}$ is the spin connection defined by the vielbein $e_{A}^{\alpha}$. As explained in [11], this vielbein defines an internal metric $g_{\alpha \beta}=e_{\alpha}^{A} e_{\beta}^{A}$, which is immanent in the spectral triple representing the D-brane configuration. Then, repeating the argument given in section 3.6.2, we obtain

$$
S_{C S}^{D(-1)}=C_{0} \int[d x][d \psi] \operatorname{Tr} \mathrm{P} \exp \left(-\int d \sigma L\left(x^{\alpha}, \psi^{\alpha}\right)\right)
$$

where

$$
L\left(x^{\alpha}, \psi^{\alpha}\right)=\frac{1}{4 u^{2}} g_{\alpha \beta}(x)\left(\dot{x}^{\alpha} \dot{x}^{\beta}+\psi^{\alpha} \nabla_{\sigma} \psi^{\beta}\right)
$$




$$
+A_{\alpha}(x) \dot{x}^{\alpha}-\frac{1}{2} F_{\alpha \beta}(x) \psi^{\alpha} \psi^{\beta}
$$

where $\nabla_{\sigma} \psi^{\alpha}=\dot{\psi}^{\alpha}+\dot{x}^{\beta} \Gamma_{\beta \gamma}^{\alpha} \psi^{\gamma}$. This system is nothing but the supersymmetric quantum mechanics which was used to derive the Atiyah-Singer index theorem in $[38,39]$.

In this case, since we are now interested in the dependence of the curvature in CSterm, we have to calculate the derivative corrections, in which the path integral with respect to non-zero modes will contribute. Fortunately, the relevant calculation was essentially done in $[38,39]$ and the result is exactly what we expect from the index theorem;

$$
S_{C S}^{D(-1)}=C_{0} \int_{D p} \widehat{A}(R) \wedge \operatorname{Tr} e^{F / 2 \pi}
$$

where $\widehat{A}(R)$ is the A-roof genus written in terms of the internal metric introduced in (3.109).

Note that above calculation $[38,39]$ is consistently done in $u \rightarrow 0$ limit, which is also taken in the heat kernel method. Since the index (3.103) does not depend on the parameter $u$, we can take this rather unphysical limit. This limit can also be thought of as the zero slope limit $\alpha^{\prime} \rightarrow 0$. If we consider the parameter $u$ as a dimensionless parameter and recover the $\alpha^{\prime}$ dependence, the D-brane configuration will become

$$
T=u \sqrt{\frac{\alpha^{\prime}}{2}}\left(\widehat{p}_{\alpha}+A_{\alpha}(\widehat{x})\right) \gamma^{\alpha}, \quad \Phi^{\alpha}=\sqrt{\frac{2}{\alpha^{\prime}}} \widehat{x}^{\alpha} .
$$

Here, we treat $T$ and $\Phi^{\alpha}$ as dimensionless operators. The normalization is fixed by requiring that the path integral representation of the operator $\widehat{x}^{\alpha}$ will have the same normalization as the closed string coordinate $X^{\alpha}(\sigma)$.

Then, (3.103) will become

$$
S_{C S}^{D(-1)}=C_{0} \operatorname{Str}\left(e^{2 \pi u^{2} \alpha^{\prime} \not D^{2}}\right)
$$

and hence the $\alpha^{\prime} \rightarrow 0$ limit plays the same role as $u \rightarrow 0$ limit in this case.

However, this is not the end of the story. The CS-term (3.74) or (3.92) that we have been using is only reliable for the lowest order in the derivative expansions. In order to fully incorporate the derivative corrections of the CS-term, we should have started from (3.72). The ascent relation for the CS-terms (3.70) is also valid including the 
derivative corrections, since it has been derived among the boundary states without any loss of information. In this case, the fluctuations of the scalar fields will contribute to the calculation of D-instanton charge density, and $\alpha^{\prime}$ cannot be absorbed in the parameter $u$ anymore. In order to obtain the fluctuation around $\mathrm{D} p$-brane solution, we have to take $u \rightarrow \infty$ keeping $\alpha^{\prime}$ finite. In the $u \rightarrow \infty$ limit, the dependence upon the vielbein $e_{A}^{\alpha}$ disappears. However, we still obtain the same formula as (3.112), where the curvature two-form $R$ with respect to the internal metric is replaced with that defined by the induced metric on the D $p$-brane world-volume. The calculation will be summarized in Appendix C. In this case, we can perform the calculation in the zero slope limit $\alpha^{\prime} \rightarrow 0$. This result is consistent with what we expect in the CS-term for the D p-brane [40]. ${ }^{17}$ (See also [27] for a related calculation.) Since the D-instanton charge is quantized, the coefficient of $C_{0}$ should not depend on the continuous parameters $u$ and $\alpha^{\prime}$, nor the choice of the metric on the $\mathrm{D} p$-brane. Therefore, the previous result (3.112) also gives a correct D-instanton charge and the interpretation of the index of the tachyon as the D-instanton charge is still valid, though it is not the case for the charge density.

We can easily extend the argument given to the index theorems which involve even or odd superconnections by considering the solution of $\mathrm{D} p-\overline{\mathrm{D} p}$ pairs or non-BPS $\mathrm{D} p$-branes, respectively. We could also start from the unstable $\mathrm{D} q$-brane system and construct $\mathrm{D}(q+p)$-branes using the ascent relation. In this case, the tachyon is the p-dimensional Dirac operator parametrized by the world volume coordinates of the unstable Dq-brane system. We can again obtain an index theorem by comparing the coupling to the RR-fields in the two equivalent descriptions. The corresponding index theorem is called family index theorem, which is related to KK-theory as expected from the result of $[11,25]$. It is also possible to generalize the argument to type I string theory, as we will discuss in section 5.3.

As we have seen in this subsection, we can think the index theorem as a topological

\footnotetext{
${ }^{17}$ In $[41,11]$ (see also [6]), the CS-term is written by Todd class $T d(R)$ instead of the A-roof genus $\widehat{A}(R)$. Here we have implicitly assumed that there is a spin structure on the world volume of the $\mathrm{D} p$-brane since we have introduced the gamma matrices on it. In this case we have $\widehat{A}(R)=T d(R)$ and there is no discrepancy. We can also treat the $\operatorname{Spin}^{c}$ cases by turning on a $U(1)$ gauge field associated with the $\operatorname{Spin}^{c}$ structure.
} 
aspect of the ascent relation among the D-brane boundary states explained in the previous subsections. In other words, we can think the D-brane ascent relations as a kind of generalization of the index theorem. Actually the essence of the derivation of the D-brane ascent relation is the equivalence between path integral and operator formulations of a supersymmetric quantum mechanics, which is also used in the physical proof of the index theorem given in $[39,38,20]$.

\subsection{Application to non-commutative gauge theory}

Before closing this section, let us make a brief comment on the application to noncommutative gauge theory. In this paper, we mainly concentrate on the fluctuations around the commutative D-brane solutions. However, our strategy is also applicable to non-commutative D-branes. In fact, it has been argued in $[42,45,43]$ that the equivalence of commutative and non-commutative descriptions of D-brane in bosonic string theory can be clearly understood using boundary states.

It would be instructive to show this is also the case for the superstring case using our superfield formulation. To be specific, let us consider a non-commutative D2-brane in type IIA string theory. The non-commutative D2-brane can be constructed from infinitely many D0-branes by turning on non-commutativity between $\Phi^{1}$ and $\Phi^{2}$ as

$$
\Phi^{\alpha}=\widehat{z}^{\alpha}, \quad(\alpha=1,2)
$$

with $\left[\widehat{z}^{\alpha}, \widehat{z}^{\beta}\right]=i \Theta^{\alpha \beta}$, where $\Theta^{\alpha \beta}=-\Theta^{\beta \alpha}$ is the real constant parameter which represent the non-commutativity.

Then the boundary interaction is given by

$$
e^{-S_{b}}=\int\left[d \mathbf{z}^{\alpha}\right] \exp \left\{-i \int d \widehat{\sigma}\left(\Theta_{\alpha \beta}^{-1} \mathbf{z}^{\alpha} D \mathbf{z}^{\beta}+\mathbf{z}^{\alpha} \mathbf{P}_{\alpha}\right)\right\}
$$

where we have introduced the superfields $\mathbf{z}^{\alpha}$ corresponding to the operators $\widehat{z}^{\alpha}$, and the non-commutativity is represented in their kinetic term. Therefore, the boundary state will become

$$
e^{-S_{b}}|B 0 ; \pm\rangle=e^{-i \int d \hat{\sigma} \Theta_{\alpha \beta}^{-1} \mathbf{X}^{\alpha} D \mathbf{X}^{\beta}}|B 2 ; \pm\rangle
$$


which is equivalent to a D2-brane boundary state with constant magnetic flux $F_{\alpha \beta}=$ $i \Theta_{\alpha \beta}^{-1}$, which can also be considered as the effect of background $B$-field $B_{\alpha \beta}=\Theta_{\alpha \beta}^{-1} \cdot[14$, $44]$

It is also easy to include the fluctuations around the solution (3.115) repeating the argument given in section 3.2 for the commutative case. The local gauge transformation of this system is given by the unitary transformation $U(\widehat{z})$ which depend on the noncommutative coordinates $\widehat{z}^{\alpha}$. Since $\widehat{z}^{\alpha}$ don't commute with $U(\widehat{z})$, we introduce the non-commutative gauge field $\widehat{A}_{\alpha}(\widehat{z})$ to maintain the local gauge invariance and replace $(3.115)$ as

$$
\Phi^{\alpha}=\widehat{z}^{\alpha}+i \Theta^{\alpha \beta} \widehat{A}_{\beta}(\widehat{z})
$$

Then, the boundary state will become

$$
\int\left[d \mathbf{z}^{\alpha}\right] \exp \left\{-i \int d \widehat{\sigma}\left(\Theta_{\alpha \beta}^{-1} \mathbf{z}^{\alpha} D \mathbf{z}^{\beta}+\left(\mathbf{z}^{\alpha}+i \Theta^{\alpha \beta} \widehat{A}_{\beta}(\mathbf{z})\right) \mathbf{P}_{\alpha}\right)\right\}|B 0 ; \pm\rangle .
$$

On the other hand, the boundary state of a D2-brane with the gauge field in the constant $B$-field background is given as

$$
\int\left[d \mathbf{x}^{\alpha}\right] \exp \left\{-i \int d \widehat{\sigma}\left(B_{\alpha \beta} \mathbf{x}^{\alpha} D \mathbf{x}^{\beta}-i A_{\alpha}(\mathbf{x}) D \mathbf{x}^{\alpha}+\mathbf{x}^{\alpha} \mathbf{P}_{\alpha}\right)\right\}|B 0 ; \pm\rangle .
$$

These two boundary states (3.119) and (3.120) are equal when $B_{\alpha \beta}=\Theta_{\alpha \beta}^{-1}$ and the gauge fields $\widehat{A}_{\alpha}$ and $A_{\alpha}$ related by the Seiberg-Witten map [46]. Actually, (3.119) and (3.120) can be obtained by simply replacing all the fields with superfields appearing in the corresponding boundary states $|\phi\rangle_{\mathrm{NC}}$ and $|\mathcal{A}\rangle$ defined in [45], respectively, and it is straightforward to generalize the argument in [45] to find the Seiberg-Witten map requiring the equality of the two boundary states (3.119) and (3.120).

\section{Generalization to higher dimensional systems}

So far, we have considered the construction of D-branes in K-matrix theory, which is the lowest dimensional unstable D-brane system. The method developed in the previous section can also be applied to the construction of D-branes in higher dimensional unstable D-brane systems. In this section, we generalize the above consideration to 
the higher dimensional unstable D-brane systems and show the general D-brane descent/ascent relations. First, we consider space-time filling unstable D-brane system and provide a simple derivation of D-brane descent relations. Then, we further generalize the argument to the construction of $\mathrm{D} p$-branes from unstable $\mathrm{D} q$-brane systems.

\subsection{D-brane descent relations}

Let us consider non-BPS D9-branes in type IIA string theory. In this case, the configuration representing $\mathrm{D} p$-branes with the fluctuations corresponding to the massless fields is expected to be

$$
T=u \gamma^{i}\left(x^{i}-\phi^{i}\left(x^{\alpha}\right)\right), \quad A_{\alpha}=A_{\alpha}\left(x^{\alpha}\right), \quad A_{i}=0
$$

where $i=p+1, \ldots, 9$ and $\alpha=0, \ldots, p[18,8]$. Here we restrict $p=$ even and consider BPS D $p$-branes for simplicity. (See the next subsection for the generalization to more general situations.) The fluctuation corresponding to the scalar fields $\phi^{i}\left(x^{\alpha}\right)$ appear in this way in the tachyon field, since they correspond to the Nambu-Goldstone modes for the spontaneous breaking of the translational symmetry $x^{i} \rightarrow x^{i}+\epsilon^{i}$.

Then, $\mathbf{M}$ in (2.16) becomes

$$
\mathbf{M}=u\left(\mathbf{X}^{i}-\phi^{i}\left(\mathbf{X}^{\alpha}\right)\right) \Gamma^{i}-A_{\alpha}\left(\mathbf{X}^{\alpha}\right) D \mathbf{X}^{\alpha}
$$

Using (2.11) and the boundary interaction of the form (2.21), we obtain the non-BPS D9 brane boundary state in the background (4.1);

$$
\begin{gathered}
|B 9 ; \pm\rangle_{S_{b}}=\int\left[d \mathbf{x}^{\mu}\right]\left[d \boldsymbol{\Gamma}^{i}\right] \operatorname{Tr} \widehat{\mathrm{P}} \exp \left\{\int d \widehat { \sigma } \left(\frac{1}{4} \boldsymbol{\Gamma}^{i} D \boldsymbol{\Gamma}^{i}+u\left(\mathbf{x}^{i}-\phi^{i}\left(\mathbf{x}^{\alpha}\right)\right) \boldsymbol{\Gamma}^{i}\right.\right. \\
\left.\left.-A_{\alpha}\left(\mathbf{x}^{\alpha}\right) D \mathbf{x}^{\alpha}-i \mathbf{x}^{\mu} \mathbf{P}_{\mu}\right)\right\}\left|\mathbf{x}^{\mu}=0 ; \pm\right\rangle
\end{gathered}
$$

where $\mu=0, \ldots, 9$ and $\left|\mathbf{x}^{\mu}=0 ; \pm\right\rangle=\left|x^{\mu}=0\right\rangle\left|\psi^{\mu}=0 ; \pm\right\rangle$. Note that the boundary action is linear with respect to $\mathbf{x}^{i}$ and the path integration of it gives $\delta\left(u \boldsymbol{\Gamma}^{i}-i \mathbf{P}_{i}\right)$. This expression is rather formal, since $\mathbf{P}_{i}$ is an operator constructed by the oscillators $\alpha_{m}^{i}$, $\widetilde{\alpha}_{m}^{i}, \Psi_{r}^{\mu}$ and $\widetilde{\Psi}_{r}^{\mu}$, while $\Gamma^{i}$ is an ordinary function. To be more precise, if we introduce a coherent state for the momentum operators which satisfy

$$
\mathbf{P}_{\mu}(\sigma)\left|\widetilde{\mathbf{p}}_{\mu} ; \pm\right\rangle=\widetilde{\mathbf{p}}_{\mu}(\sigma)\left|\widetilde{\mathbf{p}}_{\mu} ; \pm\right\rangle
$$




$$
\left|\mathbf{x}^{\mu} ; \pm\right\rangle=\int\left[d \widetilde{\mathbf{p}}_{\mu}\right] e^{-i \int d \hat{\sigma} \tilde{\mathbf{p}}_{\mu} \mathbf{x}^{\mu}}\left|\widetilde{\mathbf{p}}_{\mu} ; \pm\right\rangle
$$

the formal delta-functional and the arguments below will be justified.

Then, integrating out $\boldsymbol{\Gamma}^{i}$, we obtain

$$
\begin{gathered}
|B 9 ; \pm\rangle_{S_{b}}=\int\left[d \mathbf{x}^{\alpha}\right] \operatorname{Tr} \widehat{\mathrm{P}} \exp \left\{-\int d \widehat{\sigma}\left(\frac{1}{4 u^{2}} \mathbf{P}_{i} D \mathbf{P}_{i}+i \phi^{i}\left(\mathbf{x}^{\alpha}\right) \mathbf{P}_{i}\right.\right. \\
\left.\left.+A_{\alpha}\left(\mathbf{x}^{\alpha}\right) D \mathbf{x}^{\alpha}+i \mathbf{x}^{\alpha} \mathbf{P}_{\alpha}\right)\right\}\left|\mathbf{x}^{\mu}=0 ; \pm\right\rangle
\end{gathered}
$$

Taking $u \rightarrow \infty$ limit, we correctly obtain the the boundary state for the $\mathrm{D} p$-brane with the boundary interaction including all the massless fields on it. This gives a simple proof of the D-brane descent relations.

As we have demonstrated in section 3.6, it is straightforward to apply this result to derive the CS-term for D $p$-branes from that for an unstable D9-brane system. One of the interesting points in our derivation is that the the gamma matrices $\gamma^{i}$ are replaced with the fermionic fields $\eta^{i}(\sigma)$ (the first component of the superfield $\boldsymbol{\Gamma}^{i}(\sigma)$ ) which anticommutes with each others in the path-integral formalism. And, analogous to argument given above (3.99), the fermion $\eta^{i}(\sigma)$ are reduced to their zero-modes. Note that the

zero modes satisfy the same algebra as that for $\frac{\partial}{\partial\left(d x^{i}\right)}$ or $d x^{\alpha} ;\left\{\frac{\partial}{\partial\left(d x^{i}\right)}, \frac{\partial}{\partial\left(d x^{j}\right)}\right\}=0$, which is different from that for the gamma matrices; $\left\{\gamma^{i}, \gamma^{j}\right\} \sim \delta_{i j}$. This justifies the naive replacement of $\gamma^{i}$ to $\frac{\partial}{\partial\left(d x^{i}\right)}$ given in [47] to show the equivalence between CS-terms of the BPS D $p$-branes and the non-BPS D9-branes.

\section{2 $\mathrm{D} p$-branes from unstable $\mathrm{D} q$-brane systems}

It is now clear that combining the D-brane ascent and descent relations described above, we can construct $\mathrm{D} p$-branes from an unstable $\mathrm{D} q$-brane system with arbitrary combination of $p$ and $q$. Let us demonstrate the construction of a BPS D $p$-brane from non-BPS D $q$-branes. We consider the non-BPS D $q$-brane extended along the $x^{0}, \cdots, x^{q}$ directions. Then we construct the $\mathrm{D} p$-brane extended along $x^{\alpha}(\alpha=0, \ldots, q-m)$ and $x^{\beta}(\beta=q+1, \ldots, q+n)$ by localizing in the $m$ directions and extending in the $n$ directions, where $p=q-m+n$. The configuration representing the $\mathrm{D} p$-brane is given 
as a combination of (3.41)-(3.42) and (4.1);

$$
\begin{aligned}
T & =u \sum_{i=q-m+1}^{q}\left(x^{i}-\phi^{i}\left(x^{\alpha}, \widehat{x}^{\beta}\right)\right) \otimes \gamma^{i}+u \sum_{\beta=q+1}^{q+n}\left(\widehat{p}_{\beta}-i A_{\beta}\left(x^{\alpha}, \widehat{x}^{\beta}\right)\right) \otimes \gamma^{\beta} \\
A_{\alpha} & =A_{\alpha}\left(x^{\alpha}, \widehat{x}^{\beta}\right) \otimes 1, \quad A_{i}=0, \\
\Phi^{\beta} & =\widehat{x}^{\beta} \otimes 1, \quad \Phi^{j}=\phi^{j}\left(x^{\alpha}, \widehat{x}^{\beta}\right) \otimes 1,
\end{aligned}
$$

where the indices run as $\alpha=0, \ldots, q-m, \beta=q+1, \ldots, q+n, i=q-m+1, \ldots, q$ and $j=q+n+1, \ldots, 9$. Namely, the indices $(\alpha, i)$ and $(\beta, j)$ correspond to the directions parallel and perpendicular to the $\mathrm{D} q$-branes, respectively, while $(\alpha, \beta)$ and $(i, j)$ correspond to directions parallel and perpendicular to the $\mathrm{D} p$-branes, respectively. We will use the convention of these indices below in this subsection. Here, $\gamma^{i}$ and $\gamma^{\beta}$ form the minimally represented $S O(n+m)$ gamma matrices.

Then the boundary state becomes

$$
|B q ; \pm\rangle_{S_{b}}=\int\left[d \mathbf{x}^{\alpha}\right]\left[d \mathbf{x}^{i}\right] e^{-S_{b}} e^{-i \int d \hat{\sigma}\left(\mathbf{x}^{\alpha} \mathbf{P}_{\alpha}+\mathbf{x}^{i} \mathbf{P}_{i}\right)}\left|\mathbf{x}^{\mu}=0 ; \pm\right\rangle,
$$

where

$$
e^{-S_{b}}=\int\left[d \mathbf{x}^{\beta}\right]\left[d \mathbf{p}_{\beta}\right]\left[d \boldsymbol{\Gamma}^{\beta}\right]\left[d \boldsymbol{\Gamma}^{i}\right] \exp \left\{\int d \widehat{\sigma}\left(\frac{1}{4} \boldsymbol{\Gamma}^{\beta} D \boldsymbol{\Gamma}^{\beta}+\frac{1}{4} \boldsymbol{\Gamma}^{i} D \boldsymbol{\Gamma}^{i}+i \mathbf{p}_{\beta} D \mathbf{x}^{\beta}+\mathbf{M}\right)\right\}
$$

with

$$
\begin{aligned}
\mathbf{M}=- & i \mathbf{x}^{\beta} \mathbf{P}_{\beta}-i \phi^{j}\left(\mathbf{x}^{\alpha}, \mathbf{x}^{\beta}\right) \mathbf{P}_{j}-A_{\alpha}\left(\mathbf{x}^{\alpha}, \mathbf{x}^{\beta}\right) D \mathbf{x}^{\alpha} \\
& +u\left(\mathbf{p}_{\beta}-i A_{\beta}\left(\mathbf{x}^{\alpha}, \mathbf{x}^{\beta}\right)\right) \Gamma^{\beta}+u\left(\mathbf{x}^{i}-\phi^{i}\left(\mathbf{x}^{\alpha}, \mathbf{x}^{\beta}\right)\right) \Gamma^{i}
\end{aligned}
$$

Following the previous discussions, we perform the path-integrations $\int\left[d \boldsymbol{\Gamma}^{\beta}\right]\left[d \mathbf{p}_{\beta}\right]$ and $\int\left[d \mathbf{x}^{i}\right]\left[d \boldsymbol{\Gamma}^{i}\right]$ which forces the replacements $\boldsymbol{\Gamma}^{\beta} \rightarrow-\frac{i}{u} D \mathbf{x}^{\beta}$ and $\boldsymbol{\Gamma}^{i} \rightarrow \frac{i}{u} \mathbf{P}^{i}$, respectively. Then in the $u \rightarrow \infty$ limit we obtain

$$
|B q ; \pm\rangle_{S_{b}}=\int\left[d \mathbf{x}^{\alpha}\right]\left[d \mathbf{x}^{\beta}\right] e^{-S_{b}}\left|\mathbf{x}^{\alpha}, \mathbf{x}^{\beta}, \mathbf{x}^{i}=\mathbf{x}^{j}=0 ; \pm\right\rangle
$$

where

$$
\begin{aligned}
S_{b}=\int d & \widehat{\sigma}\left(A_{\alpha}\left(\mathbf{x}^{\alpha}, \mathbf{x}^{\beta}\right) D \mathbf{x}^{\alpha}+A_{\beta}\left(\mathbf{x}^{\alpha}, \mathbf{x}^{\beta}\right) D \mathbf{x}^{\beta}\right. \\
& \left.+i \phi^{i}\left(\mathbf{x}^{\alpha}, \mathbf{x}^{\beta}\right) \mathbf{P}_{i}+i \phi^{j}\left(\mathbf{x}^{\alpha}, \mathbf{x}^{\beta}\right) \mathbf{P}_{j}\right)
\end{aligned}
$$


Therefore we correctly obtain the boundary state for the BPS D $p$-brane with the massless fluctuations.

The construction of non-BPS D $p$-brane is obtained by adding $t\left(x^{\alpha}, \widehat{x}^{\beta}\right) \otimes \gamma_{*}$ in the tachyon configuration (4.7), where $\gamma_{*}=\prod_{i=q-m+1}^{q} \gamma^{i} \prod_{\beta=q+1}^{q+n} \gamma^{\beta}$ up to a phase factor. If we start with $\mathrm{D} q-\overline{\mathrm{D} q}$ system, $\gamma^{i}$ and $\gamma^{\beta}$ in (4.7) need not be hermitian and they are chosen such that $\Gamma^{i}=\left({ }_{\gamma^{i \dagger}} \gamma^{i}\right)$ and $\Gamma^{\beta}=\left({ }_{\gamma^{\beta \dagger}}{\gamma^{\beta}}^{\beta}\right)$ become $S O(n+m)$ gamma matrices. In this case, we have two sets of gauge fields and scalar fields which are created by $\mathrm{D} q-\mathrm{D} q$ strings and $\overline{\mathrm{D} q}-\overline{\mathrm{D} q}$ strings. But, we only turn on $A^{+}$and $\Phi_{+}$in (2.17) in the same way as given in (4.8) and (4.9). As we can see in (2.17), the other ones $A^{-}$and $\Phi_{-}$appear as the higher terms in the gamma matrix expansion and the they will not contribute in the $u \rightarrow \infty$ limit as explained in section 3.5.

\section{$5 \quad$ Generalization to type I string theory}

In this section, we generalize our argument in the previous sections to type I string theory. In the type II string theory, there are basically two types of unstable D-brane systems, namely the non-BPS D-branes and the D-brane - anti D-brane systems. Type I string has eight types of unstable D-brane systems, which are summarized in table 1. They are governed by the real Clifford algebra structure explained in the next subsection. We will see that the method using gamma matrix expansion efficiently works in these unstable D-brane systems. In this paper, we will only consider turning on the fields created by $\mathrm{D} p-\mathrm{D} p$ (or $\mathrm{D} p-\overline{\mathrm{D} p}$ ) strings and ignore the fields created by the open strings stretched between the D $p$-brane and one of the background D9-branes. ${ }^{18}$

\subsection{Hidden Clifford algebra structure}

There is an underlying Clifford algebra which characterize the structure of Chan-Paton indices for each unstable D-brane system. Recall that we used the Pauli matrices $\sigma_{1}$ and $\sigma_{2}$ to expand the matrix $\mathbf{M}$ in (2.17) for the $\mathrm{D} p-\overline{\mathrm{D} p}$ system in type II string theory. The Pauli matrices $\sigma_{1}$ and $\sigma_{2}$ generate the complex Clifford algebra $\mathbf{C}_{2}$. For non-BPS

\footnotetext{
${ }^{18}$ See [48] for the study of the type I D-branes using the boundary state formalism, including the effect of the states created by the D $p$-D9 strings.
} 
D-branes in type II theory, the matrix $\mathbf{M}$ is expanded by $\sigma_{1}$ which is the generator of $\mathbf{C}_{1}$. Here $\mathbf{C}_{n}$ is the complex Clifford algebra generated by $n$ elements $e_{1}, \ldots, e_{n}$ satisfying

$$
\left\{e_{i}, e_{j}\right\}=2 \delta_{i j}
$$

Note that, as we can see in (2.17), the tachyon fields are accompanied by odd numbers of the generators of the Clifford algebra, while the gauge fields and the scalar fields are accompanied by even numbers of the generators.

Similarly, in type I string theory, there is a hidden real Clifford algebra structure in the unstable D-brane system [25]. The real Clifford algebra $\mathbf{C}^{r, s}$ is defined as an algebra over $\mathbf{R}$ generated by $e_{i}(i=1, \ldots, r+s)$ satisfying

$$
\begin{array}{cl}
e_{i}^{2}=-1 & (i=1, \ldots, r), \\
e_{i}^{2}=+1 & (i=r+1, \ldots, r+s), \\
e_{i} e_{j}+e_{j} e_{i}=0 & (i \neq j) .
\end{array}
$$

The matrix $\mathbf{M}$ for the unstable $\mathrm{D} p$-brane system with $p=s-r+1(\bmod 8)$ in type I string theory is given by

$$
\mathbf{M}=-\widehat{A}_{\alpha}(\mathbf{X}) D \mathbf{X}^{\alpha}-i \widehat{\Phi}^{i}(\mathbf{X}) \mathbf{P}_{i}+\widehat{T}(\mathbf{X})
$$

where ${ }^{19}$

$$
\begin{aligned}
\widehat{A}_{\alpha}(\mathbf{X}) & =\sum_{w_{n} \in \mathbf{C}_{\text {even }}^{r, s}} A_{\alpha}^{(n)}(\mathbf{X}) \otimes w_{n}, \\
\widehat{\Phi}^{i}(\mathbf{X}) & =\sum_{w_{n} \in \mathbf{C}_{\text {even }}^{r, s}} \Phi_{(n)}^{i}(\mathbf{X}) \otimes w_{n}, \\
\widehat{T}(\mathbf{X}) & =\sum_{v_{n} \in \mathbf{C}_{\text {odd }}^{r, s}} T^{(n)}(\mathbf{X}) \otimes v_{n} .
\end{aligned}
$$

Here $\mathbf{C}_{\text {even }}^{r, s}$ and $\mathbf{C}_{\text {odd }}^{r, s}$ denote the subspaces of $\mathbf{C}^{r, s}$ that consist of elements which are even and odd under the involution $e_{i} \rightarrow-e_{i}$, respectively, and we sum over the basis $\left\{w_{n}\right\}$ and $\left\{v_{n}\right\}$ of the vector spaces $\mathbf{C}_{\text {even }}^{r, s}$ and $\mathbf{C}_{\text {odd }}^{r, s}$, respectively. The fields $A_{\alpha}^{(n)}, \Phi_{(n)}^{i}$ and $T^{(n)}$ in (5.4) are real matrices (or operator acting on a real Hilbert space). The generators $e_{i}$ of the Clifford algebra $\mathbf{C}^{r, s}$ are considered to be fermionic and the choice

\footnotetext{
${ }^{19}$ In this section, every tensor product is defined over $\mathbf{R}$.
} 
of $\mathbf{C}_{\text {even }}^{r, s}$ and $\mathbf{C}_{\text {odd }}^{r, s}$ in (5.4) is determined so that $\mathbf{M}$ is a fermionic superfield. We also impose the hermiticity condition $\mathbf{M}^{\dagger}=\mathbf{M}$, where the hermite conjugate of the generators of the Clifford algebra are defined as $e_{i}^{*}=-e_{i}(i=1, \ldots, r)$ and $e_{i}^{*}=e_{i}$ $(i=r+1, \ldots, r+s)$. In (5.3), D $\mathbf{X}^{\alpha}$ and $\mathbf{P}_{i}$ are considered to be anti-hermitian, since the time-like and space-like directions in the open string world-sheet is interchanged in the closed string picture. Therefore, the gauge field $\widehat{A}_{\alpha}$ is anti-hermitian, while $\widehat{\Phi}^{i}$ and $\widehat{T}$ are hermitian. It is shown in [25] that the hermiticity condition of (5.3) implies that the gauge group and the representation of the scalar fields and the tachyon field with respect to the gauge group are listed in table 1, which is consistent with the result in [49].

\begin{tabular}{c|cccc}
\hline \hline$p$ & Gauge & Tachyon & Scalar & Clifford algebra $\mathbf{C}_{D p}^{\min }$ \\
\hline-1 & $U$ & $\boxminus$ & adj. & $\mathbf{C}^{2,0}$ \\
0 & $O$ & $\boxminus$ & $\square$ & $\mathbf{C}^{1,0}$ \\
1 & $O \times O$ & $(\square, \square)$ & $(1, \square),(\square, 1)$ & $\mathbf{C}^{1,1}$ \\
2 & $O$ & $\square$ & $\square$ & $\mathbf{C}^{0,1}$ \\
3 & $U$ & $\square$ & adj. & $\mathbf{C}^{0,2}$ \\
4 & $S p$ & $\square$ & $\boxminus$ & $\mathbf{C}^{0,3}$ \\
5 & $S p \times S p$ & $(\square, \square)$ & $(1, \boxminus),(\boxminus, 1)$ & $\mathbf{C}^{0,4}$ \\
6 & $S p$ & $\boxminus$ & $\boxminus$ & $\mathbf{C}^{3,0}$ \\
7 & $U$ & $\boxminus$ & adj. & $\mathbf{C}^{2,0}$ \\
8 & $O$ & $\boxminus$ & $\square$ & $\mathbf{C}^{1,0}$ \\
9 & $O \times O$ & $(\square, \square)$ & -- & $\mathbf{C}^{1,1}$ \\
\hline
\end{tabular}

Table 1: The gauge group and the representation of the tachyon and scalar fields on the type I unstable $\mathrm{D} p$-brane system (non-BPS D $p$-branes for $p=$ $-1,0,2,3,4,6,7,8$ and $\mathrm{D} p-\overline{\mathrm{D} p}$ system for $p=1,5,9)$ and its underlying Clifford algebra. Here we listed the the Clifford algebra which corresponds to a non BPS $\mathrm{D} p$-brane or a pair of $\mathrm{D} p-\overline{\mathrm{D} p}$-branes.

In the table 1, we listed the minimal choices of the Clifford algebras which correspond to the unstable D-brane systems. Other choices of $r$ and $s$ in the algebra $\mathbf{C}^{r, s}$ with $p=s-r+1(\bmod 8)$ will represent $N$ non-BPS D $p$-branes (for $p=-1,0,2,3,4,6,7,8)$ or $N$ pairs of $\mathrm{D} p$ - $\overline{\mathrm{D} p}$-branes (for $p=1,5,9)$ with $N=2^{(r+s-m-n) / 2}$ when $\mathbf{C}_{D p}^{\min }=\mathbf{C}^{m, n}$. 
This is because there is an isomorphism $\mathbf{C}^{r, s} \simeq M_{N}\left(\mathbf{C}_{D p}^{\min }\right)=M_{N}(\mathbf{R}) \otimes \mathbf{C}_{D p}^{\min }$ which keeps the involution ${ }^{20}$ and we can interpret the $M_{N}(\mathbf{R})$ part as the Chan-Paton factor for $N$ unstable $\mathrm{D} p$-branes.

It is now straightforward to write down the boundary interaction following the argument around (2.21). First, we expand the matrix $\mathbf{M}$ in terms of the generators $e_{i}$ of the Clifford algebra $\mathbf{C}^{r, s}$ as

$$
\mathbf{M}=\sum_{k=0}^{r+s} \mathbf{M}^{I_{1} \cdots I_{k}} \otimes e_{I_{1} \cdots I_{k}}
$$

where $e_{I_{1} \cdots I_{k}}$ denote the skew-symmetric product of $e_{I_{1}}, \ldots, e_{I_{k}}$. Then, our proposal of the boundary interaction for the type I unstable $\mathrm{D} p$-brane is

$$
\begin{aligned}
& e^{-S_{b}}=\int\left[d \boldsymbol{\Gamma}^{I}\right] \operatorname{Tr} \widehat{\mathrm{P}} \exp \left\{\int d \widehat { \sigma } \left(-\frac{1}{4} \sum_{i=1}^{r} \boldsymbol{\Gamma}^{i} D \boldsymbol{\Gamma}^{i}+\frac{1}{4} \sum_{i=r+1}^{r+s} \boldsymbol{\Gamma}^{i} D \boldsymbol{\Gamma}^{i}\right.\right. \\
& \left.\left.+\sum_{k=0}^{r+s} \mathbf{M}^{I_{1} \cdots I_{k}} \boldsymbol{\Gamma}^{I_{1}} \cdots \boldsymbol{\Gamma}^{I_{k}}\right)\right\} .
\end{aligned}
$$

\subsection{D-branes in type I K-matrix theory}

Let us generalize the construction of D-branes given in section 3 to the case of type I string theory. To be specific, we consider D-branes in the matrix theory based on non-BPS D-instantons in type I string theory, which we call type I K-matrix theory.

As listed in table 1, the underlying Clifford algebra for the non-BPS D-instanton system is $\mathbf{C}^{2,0}$. The Clifford algebra $\mathbf{C}^{2,0}$ is faithfully represented by $e_{1}=i \sigma_{1}$ and $e_{2}=i \sigma_{2}$. Then, the tachyon part (the second term) of (5.3) is of the form

$$
\widehat{T}=T^{(1)} \otimes e_{1}+T^{(2)} \otimes e_{2}=\left(\begin{array}{cc}
T \\
-T^{*}
\end{array}\right),
$$

where we have defined $T=T^{(2)}+i T^{(1)}$ and its complex conjugate $T^{*}=T^{(2)}-i T^{(1)}$. In addition, the hermiticity condition $F^{\dagger}=F$ implies $T=-T^{T}$. The scalar part of $(5.3)$ is

$$
\widehat{\Phi}^{\mu}=\Phi_{(1)}^{\mu} \otimes 1+\Phi_{(2)}^{\mu} \otimes e_{1} e_{2}=\left(\begin{array}{cc}
\Phi^{\mu} & \\
& \Phi^{\mu *}
\end{array}\right),
$$

\footnotetext{
${ }^{20}$ In order to keep the involution, every generator $e_{i}$ of $\mathbf{C}^{r, s}$ should be mapped to an element in $M_{N}(\mathbf{R}) \otimes \mathbf{C}_{\text {odd }}^{m, n}$, i.e. the odd elements of $M_{N}\left(\mathbf{C}^{m, n}\right)$.
} 
where $\Phi^{\mu}=\Phi_{(1)}^{\mu}-i \Phi_{(2)}^{\mu}$ and $\Phi^{\mu *}=\Phi_{(1)}^{\mu}+i \Phi_{(2)}^{\mu}$, and the hermiticity condition implies $\Phi^{\mu \dagger}=\Phi^{\mu}$. Here $T$ and $\Phi^{\mu}$ are the fields on the non-BPS D-instantons, which are operators acting on a Hilbert space.

The Dp-brane solution in type I K-matrix theory is constructed in [25] as

$$
\begin{aligned}
\widehat{T} & =\left(\begin{array}{c}
T^{\dagger} \\
T^{\prime}
\end{array}\right)=u\left(\widehat{\partial}_{0} e_{1}+\sum_{\alpha=1}^{p} \widehat{\partial}_{\alpha} \gamma_{p}^{\alpha} e_{2}\right) \\
\Phi^{\alpha} & =\widehat{x}^{\alpha} \quad(\alpha=0, \ldots, p), \quad \Phi^{i}=0 \quad(i=p+1, \ldots, 9),
\end{aligned}
$$

where $\widehat{\partial}_{\alpha}=\partial / \partial x^{\alpha}$ and $\widehat{x}^{\alpha}$ are the operators acting on a real Hilbert space $L^{2}\left(\mathbf{R}^{p+1}\right)$ and $\gamma_{p}^{\alpha}$ are the $S O(p)$ gamma matrices represented as real symmetric matrices. $u$ is a real parameter and $u \rightarrow \infty$ gives the exact solution. Note that the tachyon operator (5.9) can be rewritten as

$$
\widehat{T}=u \sum_{\alpha=0}^{p} \widehat{\partial}_{\alpha} \widehat{\Gamma}^{\alpha}
$$

where we have set $\widehat{\Gamma}^{0}=e_{1}$ and $\widehat{\Gamma}^{\alpha}=\gamma_{p}^{\alpha} e_{2} \quad(\alpha=1, \ldots, p)$. These $\widehat{\Gamma}^{\alpha}$ satisfies

$$
\left\{\widehat{\Gamma}^{\alpha}, \widehat{\Gamma}^{\beta}\right\}=-2 \delta^{\alpha \beta}, \quad(\alpha, \beta=0, \ldots, p)
$$

And hence it can be thought of as a natural analog of the $\mathrm{D} p$-brane solution (3.4) in type II string theory. These $\widehat{\Gamma}^{\alpha}$ are elements of $M_{n_{p}}\left(\mathbf{C}^{2,0}\right)$, where $n_{p}$ is the size of the gamma matrices $\gamma_{p}^{\alpha}$ listed in table 2 . We do not have to stick to the explicit

\begin{tabular}{c|cccccccccc}
\hline \hline$p$ & 0 & 1 & 2 & 3 & 4 & 5 & 6 & 7 & 8 & 9 \\
\hline$n_{p}$ & 1 & 1 & 2 & 4 & 8 & 8 & 16 & 16 & 16 & 16 \\
\hline
\end{tabular}

Table 2: The size of the matrices $\gamma^{\alpha}$ which is used in the tachyon configuration (5.9) representing type I D $p$-branes.

form given in (5.9). $\left\{\widehat{\Gamma}^{\alpha}\right\}$ can be replaced by any set of odd elements which realize the commutation relation (5.12) for the $\mathbf{C}^{p+1,0}$ generators. Therefore, the D-brane configurations are obtained by choosing a homomorphism $\mathbf{C}^{p+1,0} \rightarrow M_{n_{p}}\left(\mathbf{C}^{2,0}\right)$. For 
$p=1,5$ or 9 , there are two homomorphism which are not unitary equivalent to each other, which correspond to a $\mathrm{D} p$-brane or an $\overline{\mathrm{D} p}$-brane.

In [25], it has been shown that this solution exactly gives a correct tension for a $\mathrm{D} p$ brane (BPS D $p$-brane for $p=1,5,9$ and non-BPS D $p$-brane for $p=-1,0,2,3,4,6,7,8$ ) in type I string theory and the stability analysis of the solutions consistently reproduces the spectrum of stable D-branes expected from K-theory analysis [7]. Here, we would like to show that the fluctuations around the $\mathrm{D} p$-brane solution which correspond to the gauge, scalar and tachyon fields on the $\mathrm{D} p$-brane are precisely what we expect from the table 1, by generalizing the argument given in section 3 to type I string theory. For this, we should reproduce not only the boundary state representing $\mathrm{D} p$-brane but also the correct field content of the theory, i.e., real Clifford algebra structure $\mathbf{C}_{D p}^{\min }$. We will consider $p \neq 1,5,9$ cases, though the following argument can also be applied to a $\mathrm{D} p-\overline{\mathrm{D} p}$ pair with $p=1,5,9$, if we replace $n_{p}$ with $2 n_{p}$. Then, one can show that there is an isomorphism

$$
\varphi: \mathbf{C}^{p+1+m, n} \simeq M_{n_{p}}\left(\mathbf{C}^{2,0}\right)
$$

where $m$ and $n$ are taken such that $\mathbf{C}_{D p}^{\min }=\mathbf{C}^{m, n}$. Therefore, we can realize $\widehat{\Gamma}^{\alpha}$ in $M_{n_{p}}\left(\mathbf{C}^{2,0}\right)$ by setting $\widehat{\Gamma}^{\alpha} \equiv \varphi\left(e_{\alpha+1}\right),(\alpha=0, \ldots, p)$. We extend this definition to span all the generators of $\mathbf{C}^{p+1+m, n}$ as $\widehat{\Gamma}^{I} \equiv \varphi\left(e_{I+1}\right),(I=0, \ldots, p+m+n)$. We also use the notation $\widehat{e}_{k} \equiv \widehat{\Gamma}^{p+k},(k=1, \ldots, m+n)$ later. Note that these $\widehat{e}_{k}$ give a realization of $\mathbf{C}_{D p}^{\min }=\mathbf{C}^{m, n}$ in $M_{n_{p}}\left(\mathbf{C}^{2,0}\right)$.

Using these gamma matrices $\widehat{\Gamma}^{I}$, we expand the matrix $\mathbf{M}$ as

$$
\mathbf{M}=u \sum_{\alpha=0}^{p} \widehat{\partial}_{\alpha} \widehat{\Gamma}^{\alpha}-i \sum_{\alpha=0}^{p} \widehat{x}^{\alpha} \mathbf{P}_{\alpha}+\sum_{k=0}^{p+m+n} \delta \mathbf{M}^{I_{1} \cdots I_{k}} \otimes \widehat{\Gamma}^{I_{1} \cdots I_{k}}
$$

where $\delta \mathbf{M}^{I_{1} \cdots I_{k}}$ represent the fluctuations around the solution. The boundary interaction is given as (5.6)

$$
\begin{aligned}
e^{-S_{b}}=\int\left[d \widehat{\boldsymbol{\Gamma}}^{I}\right][ & \left.d \mathbf{x}^{\alpha}\right]\left[d \mathbf{p}_{\alpha}\right] \exp \left\{\int d \widehat { \sigma } \left(-\frac{1}{4} \sum_{I=0}^{p+m} \widehat{\boldsymbol{\Gamma}}^{I} D \widehat{\boldsymbol{\Gamma}}^{I}+\frac{1}{4} \sum_{I=p+m+1}^{p+m+n} \widehat{\boldsymbol{\Gamma}}^{I} D \widehat{\boldsymbol{\Gamma}}^{I}\right.\right. \\
& \left.\left.+\sum_{\alpha=0}^{p}\left(i \mathbf{p}_{\alpha} D \mathbf{x}^{\alpha}+i u \mathbf{p}_{\alpha} \widehat{\boldsymbol{\Gamma}}^{\alpha}-i \mathbf{x}^{\alpha} \mathbf{P}_{\alpha}\right)+\sum_{k=0}^{p+m+n} \delta \mathbf{M}^{I_{1} \cdots I_{k}} \widehat{\boldsymbol{\Gamma}}^{I_{1}} \cdots \widehat{\boldsymbol{\Gamma}}^{I_{k}}\right)\right\} .
\end{aligned}
$$


Just like (3.48), the path integral with respect to $\mathbf{p}_{\alpha}$ implies

$$
D \mathbf{x}^{\alpha}+u \widehat{\boldsymbol{\Gamma}}^{\alpha}=0 .
$$

Then, following the argument leading (3.60) and (3.62), we obtain the relevant fluctuations which correspond to the gauge, scalar and tachyon fields:

$$
\begin{aligned}
\widehat{T} & =u \sum_{\alpha=0}^{p}\left(\widehat{\partial}_{\alpha}+A_{\alpha}(\widehat{x})\right) \widehat{\Gamma}^{\alpha}+t(\widehat{x}), \\
\Phi^{i} & =\phi^{i}(\widehat{x}) \quad(i=p+1, \ldots, 9) .
\end{aligned}
$$

Here $A_{\alpha}(\widehat{x}), t(\widehat{x})$ and $\phi^{i}(\widehat{x})$ are matrices which can be expanded by $\widehat{e}_{k}=\widehat{\Gamma}^{p+k},(k=$ $1, \ldots, m+n)$.

Recall that $\widehat{e}_{k}$ can be thought of as the generators of $\mathbf{C}_{D p}^{\min }$. Since the operator $\widehat{T}$ is odd under the involution $e_{i} \rightarrow-e_{i}, A_{\alpha}(\widehat{x})$ and $t(\widehat{x})$ should be even and odd elements of $\mathbf{C}_{D p}^{\min }$, respectively. Furthermore, from the hermiticity condition $\widehat{T}^{\dagger}=\widehat{T}, A_{\alpha}(\widehat{x})$ and $t(\widehat{x})$ should be anti-hermite and hermite operators, respectively. Similarly, $\phi^{i}(\widehat{x})$ are hermite operators and even elements of $\mathbf{C}_{D p}^{\min }$. These properties are exactly what we have imposed for the fields on the type I $\mathrm{D} p$-brane to obtain the table 1. Inserting (5.17) and (5.18) in (5.15) and performing the path integral with respect to $\mathbf{p}_{\alpha}$, we correctly obtain the boundary state for $\mathrm{D} p$-brane with boundary interaction (5.3) in the limit $u \rightarrow \infty$.

\subsection{CS-term, real superconnections and the index theorem}

It is interesting to apply the arguments given in section 3.6 to the type I case. The CS-term for unstable $\mathrm{D} p$-branes in type I string theory can also be written like (3.74), where (3.77) is now given as

$$
\begin{gathered}
J\left(x^{\alpha}, k_{i}, \psi_{1}^{\alpha}, \psi_{2}^{i}\right)=\operatorname{Str}\left(e^{-i k_{i} \widehat{\Phi}^{i}+2 \pi \widehat{\mathcal{F}}}\right) \\
\widehat{\mathcal{F}}=\frac{1}{2} \widehat{F}_{\alpha \beta} \psi_{1}^{\alpha} \psi_{1}^{\beta}-\widehat{T}^{2}+\frac{1}{8 \pi^{2}}\left[\widehat{\Phi}^{i}, \widehat{\Phi}^{j}\right] \psi_{2}^{i} \psi_{2}^{j}-\frac{1}{2 \pi} \psi_{1}^{\alpha} \psi_{2}^{i} D_{\alpha} \widehat{\Phi}^{i}+i \psi_{1}^{\alpha} D_{\alpha} \widehat{T}-\frac{i}{2 \pi} \psi_{2}^{i}\left[\widehat{\Phi}^{i}, \widehat{T}\right],
\end{gathered}
$$


using the notation in (5.4) and $D_{\alpha}=\partial_{\alpha}+\widehat{A}_{\alpha}$. Str in (5.19) denotes the supertrace which is proportional to the trace of the coefficient of $e_{1} e_{2} \cdots e_{r+s} . \widehat{\mathcal{F}}$ can also be written as

$$
\widehat{\mathcal{F}}=-Z^{2}, \quad Z=-i \psi_{1}^{\alpha}\left(\partial_{\alpha}+\widehat{A}_{\alpha}\right)+\frac{i}{2 \pi} \psi_{2}^{i} \widehat{\Phi}^{i}+\widehat{T} .
$$

This $\widehat{\mathcal{F}}$ is a analog of the field strength of the superconnection. Recall that there are two types of (complex) superconnections [35] based on complex Clifford algebras, called even and odd superconnections, and they naturally appear in the CS-terms of D-brane - anti D-brane systems and non-BPS D-branes [20, 21, 34], as we have seen in section 3.6.1. Generalizing the definition of the superconnection using real Clifford algebra, we can define eight types of real superconnections associated with the eight types of unstable D-brane systems listed in table 1 . When we turn off the scalar fields $\widehat{\Phi}^{i}$, the CS-term is simplified as [25]

$$
\begin{aligned}
S_{C S}^{D p} & =\mu_{p} \int_{D p} C \wedge \operatorname{Str} e^{2 \pi \widehat{\mathcal{F}}} \\
\widehat{\mathcal{F}} & =-\widehat{T}^{2}+i D_{\alpha} \widehat{T} d x^{\alpha}+\frac{1}{2} \widehat{F}_{\alpha \beta} d x^{\alpha} \wedge d x^{\beta} .
\end{aligned}
$$

We will now show that only $n$-form part of $e^{\widehat{\mathcal{F}}}$ with $n \equiv p-1(\bmod 4)$ will survive after taking the supertrace. This is consistent with the fact that the $\mathrm{R}-\mathrm{R} p$-form fields $C_{p}$ only exist for $p=2,6,(10)$ in type I string theory. ${ }^{21}$ First, note that the $n$-form part of $e^{\widehat{\mathcal{F}}}$ which contributes in the supertrace is of the form

$$
H \equiv i^{n} G e_{1} e_{2} \cdots e_{r+s} d x^{1} \cdots d x^{n}
$$

where $G$ is a real matrix. Since $\widehat{\mathcal{F}}$ is required to be hermite and bosonic, $H$ is also hermite and $r+s+n \equiv 2 k$ is an even number. Here $d x^{\alpha}$ is treated as a fermion since it came from $\psi_{1}^{\alpha}$ in (5.20). Then, hermite conjugate of $H$ is given by

$$
\begin{aligned}
H^{\dagger} & =(-1)^{\frac{1}{2}(r+s+n)(r+s+n-1)-r-n} i^{n} G^{T} e_{1} e_{2} \cdots e_{r+s} d x^{1} \cdots d x^{n} \\
& =(-1)^{k-r-n} i^{n} G^{T} e_{1} e_{2} \cdots e_{r+s} d x^{1} \cdots d x^{n} .
\end{aligned}
$$

Therefore the hermiticity of $H$ implies

$$
G=(-1)^{k-r-n} G^{T}
$$

\footnotetext{
${ }^{21}$ The coupling of R-R 10 -form is canceled by the contribution from the $O 9^{-}$-plane.
} 
Since the supertrace of $e^{\widehat{\mathcal{F}}}$ is proprotional to the trace over $G, k-r-n$ should be an even number to obtain a non-zero contribution. Then we obtain $n \equiv s-r \equiv p-1$ $(\bmod 4)$.

It is straightforward to generalize the argument given in section 3.6.2 and 3.6.3 to show the descent/ascent relations of the CS-terms for the type I D-branes and to obtain index theorems for the gauge theories listed in the table 1.

As an example, let us start with D1- $\overline{\mathrm{D} 1}$ system and consider the homogeneous configuration along the world-volume of the D1-branes. The D1-brane charge of the system can be read from the coefficient of R-R 2-form field $C_{2}$ in the CS-term and it is again given by the index of the tachyon operator on the D1- $\overline{\mathrm{D} 1}$ system. When we construct $\mathrm{D} p$-branes in this system, the tachyon operator becomes a Dirac operator for the gauge theory on the $\mathrm{D} p$-brane. On the other hand, from the viewpoint of the $\mathrm{D} p$-brane world-volume gauge theory, the D1-brane charge is given as the integral of $\operatorname{Str} e^{\widehat{\mathcal{F}}}$ along the direction transverse to the D1-brane in the D $p$-brane world-volume. Comparing the two descriptions, we conclude that the index of the Dirac operator is given by $\int \operatorname{Str} e^{\widehat{\mathcal{F}}}$.

We can also apply this strategy for the $\mathbf{Z}_{2}$ charged stable non-BPS D-branes in type I string theory found in [5,7]. As shown in [7], the D-brane charge is classified by the real K-theory. More specifically, flat $\mathrm{D} p$-branes in the flat space-time are classified by real K-theory group $K O\left(\mathbf{R}^{9-p}\right)$, which is $\mathbf{Z}$ for $p=1,5,9$ and $\mathbf{Z}_{2}$ for $p=-1,0,7,8$. These $\mathbf{Z}_{2}$ charges for the $\mathrm{D}(-1), 0,7,8$-branes cannot be written as the integral of a differential form unlike the $\mathbf{Z}$ charge of D1, 5, 9-branes. However, it is still possible to interpret the charge in terms of the configurations of tachyon and gauge fields on D9-D9 system [7] or other higher dimensional unstable D-brane systems [49] in type I string theory. On the other hand, as explained in [25], the $\mathbf{Z}_{2}$ charge of unstable D-branes are also obtained as an index of the tachyon operators. In fact, as we can see from the table 1 , the tachyon of the $\mathrm{D}(-1), 0,7,8$-branes transform as the anti-symmetric tensor representation of the gauge group, ${ }^{22}$ and hence the $\bmod 2 \operatorname{index}, \operatorname{Index} T \equiv \operatorname{dim} \operatorname{Ker} T$ $(\bmod 2)$, is invariant under small perturbations of the operator T. Again, comparing

\footnotetext{
${ }^{22}$ The tachyon on the D6-brane is also anti-symmetric. But, the anti-symmetric tensor representation of $S p$ group does not carry the index.
} 
the two descriptions, we can obtain the relations between the mod 2 indices of Dirac operators and the field configurations of the gauge theory on the higher dimensional unstable D-brane systems.

We will not study these issues further in this paper. It would be very interesting to explore the descent/ascent relations of various topological invariants more extensively using this approach.

\section{Conclusions and Discussions}

In this paper, we gave a simple derivation of D-brane descent/ascent relations. Taking the fluctuations around a D-brane solutions into consideration, we correctly reproduced the D-brane boundary state with boundary interaction. We found that the superfield formulation was very useful for the analysis. We analyzed type II as well as type I string theory. The Clifford algebra structure is efficiently used in the analysis of type I D-branes.

Since we have seen how to realize the D-brane action from K-matrix theory, we can in principle analyze anything which have been done using D-brane action in the framework of K-matrix theory. For example, since we can also obtain non-BPS D-brane action from K-matrix theory, it is possible to repeat the analysis given in [50, 51] for the rolling of the tachyon field on the non-BPS D-brane using K-matrix theory.

It is important to note that K-matrix theory makes it possible to construct any configurations of D-branes with various dimensions in a single set-up. We can also handle creation and annihilation of unstable D-branes which plays an important role in string theory. We haven't analyzed fundamental strings in K-matrix theory. But, since the fundamental strings can be realized as electric flux tubes in unstable D-brane system $[52,53,54]$, it should be possible to realize them as such configurations in Kmatrix theory. Therefore, it is reasonable to expect that the theory contains the degrees of freedom of fundamental strings as well. It would be interesting to investigate this further.

Another interesting question is how supersymmetry of the BPS D-branes constructed from the non BPS D-branes is realized. There should be a nonlinear super- 
symmetry on the unstable D-brane system and its restoration is expected to explain the BPS property $[29,55,56]$. It may be interesting to study it using the boundary states.

The field variables in K-matrix theory consists of operators acting on an infinite dimensional Hilbert space. If we regularize the theory by replacing the operators with finite size matrices, it provides a kind of lattice regularization of gauge theory on D-branes. It would be interesting to see if this approach could give a constructive definition of higher dimensional gauge theory or string theory.

As for the action of K-matrix theory, we adopted BSFT action in this paper. It is an excellent approach for the consideration of tachyon condensation. However, there are some problems concerned with massive modes [57]. In order to discuss quantum effects, it is inevitable to include these massive modes. We leave these issues for the future investigation.

\section{Acknowledgments}

We would like to thank K. Hashimoto for useful discussions. S.T. is grateful to the Michigan center for theoretical physics and the Harvard high energy theory group for hospitality. S.S. also thanks high energy theory groups at Yukawa institute for theoretical physics and Seoul national university for hospitality and especially grateful to Soo-Jong Rey for various enjoyable discussions. The work of A.T. is supported in part by JSPS Research Fellowships for Young Scientists. The work of S.S. is supported in part by Danish Natural Science Research Council.

\section{A Operator vs. path integral}

In this appendix, we will show the equivalence between operator and path integral formulations in the superfield formalism used in section 3. We will compare the following two objects.

$$
\begin{aligned}
& Z_{1}=\operatorname{Tr} \widehat{\mathrm{P}} e^{\int d \hat{\sigma} \mathbf{M}(\hat{x}, \hat{p})} \\
& Z_{2}=\int[d \mathbf{x}][d \mathbf{p}] \exp \left\{\int d \widehat{\sigma}(i \mathbf{p} D \mathbf{x}+\mathbf{M}(\mathbf{x}, \mathbf{p}))\right\}
\end{aligned}
$$


As explained in (2.14), the supersymmetric path ordered exponential in $Z_{1}$ is defined as

$$
\begin{aligned}
& Z_{1}=\sum_{n=0}^{\infty} \int d \widehat{\sigma}_{1} \cdots d \widehat{\sigma}_{n} \Theta\left(\widehat{\sigma}_{12}\right) \Theta\left(\widehat{\sigma}_{23}\right) \cdots \Theta\left(\widehat{\sigma}_{n-1 n}\right) \\
& \times(-1)^{\frac{n(n-1)}{2}} \operatorname{Tr}\left\{\mathbf{M}(\hat{x}, \hat{p})_{\hat{\sigma}_{1}} \cdots \mathbf{M}(\hat{x}, \hat{p})_{\hat{\sigma}_{n}}\right\},
\end{aligned}
$$

where $\mathbf{M}(\hat{x}, \hat{p})_{\hat{\sigma}_{i}}$ denotes $\mathbf{M}(\hat{x}, \hat{p})$ evaluated at $\hat{\sigma}=\widehat{\sigma}_{i}$. If we rewrite this formula using the standard notations for non-commutative field theory [58], we obtain

$$
\begin{aligned}
Z_{1}=\sum_{n=0}^{\infty} & \int d \widehat{\sigma}_{1} \cdots d \widehat{\sigma}_{n} \Theta\left(\widehat{\sigma}_{12}\right) \Theta\left(\widehat{\sigma}_{23}\right) \cdots \Theta\left(\widehat{\sigma}_{n-1 n}\right) \\
& \times(-1)^{\frac{n(n-1)}{2}} \frac{1}{2 \pi} \int d x d p \mathbf{M}(x, p)_{\hat{\sigma}_{1}} * \cdots * \mathbf{M}(x, p)_{\hat{\sigma}_{n}}
\end{aligned}
$$

where $*$ is the star product defined as

$$
A(x, p) * B(x, p)=\left.e^{\frac{i}{2}\left(\frac{\partial}{\partial x_{1}} \frac{\partial}{\partial p_{2}}-\frac{\partial}{\partial p_{1}} \frac{\partial}{\partial x_{2}}\right)} A\left(x_{1}, p_{1}\right) B\left(x_{2}, p_{2}\right)\right|_{\substack{x=x_{1}=x_{2} \\ p=p_{1}=p_{2}}}
$$

To compare $Z_{1}$ and $Z_{2}$ order by order, we also expand $Z_{2}$ as

$$
Z_{2}=\sum_{n=0}^{\infty} \frac{1}{n !} \int d \widehat{\sigma}_{1} \cdots d \widehat{\sigma}_{n}(-1)^{\frac{n(n-1)}{2}} \int[d \mathbf{x}][d \mathbf{p}] e^{i \int d \hat{\sigma} \mathbf{p} D \mathbf{x}} \mathbf{M}(\mathbf{x}, \mathbf{p})_{\hat{\sigma}_{1}} \cdots \mathbf{M}(\mathbf{x}, \mathbf{p})_{\hat{\sigma}_{n}}
$$

The path integral can be evaluated by the usual technique of perturbation theory as

$$
\begin{aligned}
& \int[d \mathbf{x}][d \mathbf{p}] e^{i \int d \hat{\sigma} \mathbf{p} D \mathbf{x}} \mathbf{M}(\mathbf{x}, \mathbf{p})_{\hat{\sigma}_{1}} \cdots \mathbf{M}(\mathbf{x}, \mathbf{p})_{\hat{\sigma}_{n}} \\
= & \left.\frac{1}{2 \pi} \int d x_{0} d p_{0} e^{\frac{\delta}{\delta \mathbf{p}} \cdot \Theta \frac{\delta}{\delta \mathbf{x}}} \mathbf{M}(\mathbf{x}, \mathbf{p})_{\hat{\sigma}_{1}} \cdots \mathbf{M}(\mathbf{x}, \mathbf{p})_{\hat{\sigma}_{n}}\right|_{\substack{\mathbf{x}(\hat{\sigma})=x_{0} \\
\mathbf{p}(\hat{\sigma})=p_{0}}},
\end{aligned}
$$

where we have defined

$$
\begin{aligned}
\frac{\delta}{\delta \mathbf{p}} \cdot \Theta \frac{\delta}{\delta \mathbf{x}} & =\frac{i}{4} \int d \widehat{\sigma}_{a} \int d \widehat{\sigma}_{b} \operatorname{sign}\left(\widehat{\sigma}_{a b}\right)\left(\frac{\delta}{\delta \mathbf{x}\left(\widehat{\sigma}_{a}\right)} \frac{\delta}{\delta \mathbf{p}\left(\widehat{\sigma}_{b}\right)}-\frac{\delta}{\delta \mathbf{p}\left(\widehat{\sigma}_{a}\right)} \frac{\delta}{\delta \mathbf{x}\left(\widehat{\sigma}_{b}\right)}\right) \\
& =\frac{i}{2} \int d \widehat{\sigma}_{a} \int d \widehat{\sigma}_{b} \Theta\left(\widehat{\sigma}_{a b}\right)\left(\frac{\delta}{\delta \mathbf{x}\left(\widehat{\sigma}_{a}\right)} \frac{\delta}{\delta \mathbf{p}\left(\widehat{\sigma}_{b}\right)}-\frac{\delta}{\delta \mathbf{p}\left(\widehat{\sigma}_{a}\right)} \frac{\delta}{\delta \mathbf{x}\left(\widehat{\sigma}_{b}\right)}\right)
\end{aligned}
$$

where $\operatorname{sign}\left(\widehat{\sigma}_{a b}\right)=\Theta\left(\widehat{\sigma}_{a b}\right)-\Theta\left(\widehat{\sigma}_{b a}\right)$ is the sign function. The normalization factor $1 / 2 \pi$ in (A.8) comes from the path integral measure which will be fixed in Appendix B. 
(A.8) is computed by contracting $\mathbf{x}$ and $\mathbf{p}$ with the propagator. Here, the choice of the constant term in the propagator does not affect the final result and the expression (A.8) has no ambiguity. We will use $\Theta\left(\widehat{\sigma}_{a b}\right)$ as the propagator which turns out to be very convenient in order to show $Z_{1}=Z_{2}$.

When we compute (A.8) by contracting $\mathbf{x}$ and $\mathbf{p}$ with the propagators, we encounter products of $\Theta\left(\widehat{\sigma}_{a b}\right)$ 's. However, since $\Theta\left(\widehat{\sigma}_{a b}\right)$ is defined as

$$
\begin{aligned}
\Theta\left(\widehat{\sigma}_{a b}\right) & =\Theta\left(\sigma_{a}-\sigma_{b}-\theta_{a} \theta_{b}\right) \\
& =\Theta\left(\sigma_{a}-\sigma_{b}\right)-\theta_{a} \theta_{b} \delta\left(\sigma_{a}-\sigma_{b}\right),
\end{aligned}
$$

we have to deal with the products of the delta function and step functions, which are not well-defined. In order to fix this ambiguity, we modify the step function $\Theta\left(\widehat{\sigma}_{i j}\right)$ in (A.9) as

$$
\Theta_{\epsilon}(x)=\left\{\begin{array}{cc}
0 & x<-\epsilon \\
\frac{1}{2}+\frac{x}{2 \epsilon} & -\epsilon \leq x \leq \epsilon \\
1 & \epsilon<x
\end{array}\right.
$$

and take the limit $\epsilon \rightarrow+0$ afterward. Then, the product of a step function and a delta function is understood as $\Theta\left(\sigma_{a}-\sigma_{b}\right) \delta\left(\sigma_{a}-\sigma_{b}\right)=\frac{1}{2} \delta\left(\sigma_{a}-\sigma_{b}\right)$. It is easy to check the identities

$$
\begin{aligned}
\Theta\left(\widehat{\sigma}_{a b}\right)^{2} & =\Theta\left(\widehat{\sigma}_{a b}\right), \\
\Theta\left(\widehat{\sigma}_{a b}\right) \Theta\left(\widehat{\sigma}_{b a}\right) & =0, \\
\Theta\left(\widehat{\sigma}_{a b}\right) \Theta\left(\widehat{\sigma}_{b c}\right) \Theta\left(\widehat{\sigma}_{a c}\right) & =\Theta\left(\widehat{\sigma}_{a b}\right) \Theta\left(\widehat{\sigma}_{b c}\right),
\end{aligned}
$$

etc., which are satisfied up to some functions with measure zero support.

Note also that we have

$$
\sum_{s \in S_{n}} \Theta\left(\widehat{\sigma}_{s(1) s(2)}\right) \cdots \Theta\left(\widehat{\sigma}_{s(n-1) s(n)}\right)=1,
$$

where $S_{n}$ is the permutation group of $n$ indices. From (A.15), we can replace the $\widehat{\sigma}$ integral in (A.6) as

$$
\begin{aligned}
\frac{1}{n !} \int d \widehat{\sigma}_{1} \cdots d \widehat{\sigma}_{n} & =\frac{1}{n !} \sum_{s \in S_{n}} \int d \widehat{\sigma}_{1} \cdots d \widehat{\sigma}_{n} \Theta\left(\widehat{\sigma}_{s(1) s(2)}\right) \cdots \Theta\left(\widehat{\sigma}_{s(n-1) s(n)}\right) \\
& \rightarrow \int d \widehat{\sigma}_{1} \cdots d \widehat{\sigma}_{n} \Theta\left(\widehat{\sigma}_{12}\right) \cdots \Theta\left(\widehat{\sigma}_{n-1 n}\right)
\end{aligned}
$$


using the permutation invariance of $d \widehat{\sigma}_{1} \mathbf{M}_{\hat{\sigma}_{1}} \cdots d \widehat{\sigma}_{n} \mathbf{M}_{\hat{\sigma}_{n}}$ in (A.6). Then, using the identities among the propagators such as (A.12)-(A.14), the contractions with the propagators $\Theta\left(\widehat{\sigma}_{i j}\right)$ in (A.8) can be reduced to the star products.

$$
\begin{aligned}
& \left.\Theta\left(\widehat{\sigma}_{12}\right) \cdots \Theta\left(\widehat{\sigma}_{n-1 n}\right) \int d x_{0} d p_{0} e^{\frac{\delta}{\delta \mathbf{p}} \cdot \Theta \frac{\delta}{\delta \mathbf{x}}} \mathbf{M}(\mathbf{x}, \mathbf{p})_{\hat{\sigma}_{1}} \cdots \mathbf{M}(\mathbf{x}, \mathbf{p})_{\hat{\sigma}_{n}}\right|_{\substack{\mathbf{x}(\hat{\sigma})=x_{0} \\
\mathbf{p}(\hat{\sigma})=p_{0}}} \\
= & \Theta\left(\widehat{\sigma}_{12}\right) \cdots \Theta\left(\widehat{\sigma}_{n-1 n}\right) \int d x d p \mathbf{M}(x, p)_{\sigma_{1}} * \cdots * \mathbf{M}(x, p)_{\sigma_{n}}
\end{aligned}
$$

Here we have used the fact that there is no contraction between the $\mathbf{x}$ and $\mathbf{p}$ in the same $\mathbf{M}(\mathbf{x}, \mathbf{p})_{\hat{\sigma}_{i}}$ factor. Inserting this into (A.6) and comparing it with (A.4), we obtain the desired equivalence $Z_{1}=Z_{2}$.

We can easily generalize this argument to the analogous statement for fermionic boundary super fields. Let us now show $Z_{1}=Z_{2}$, where

$$
\begin{aligned}
& Z_{1}=\kappa \operatorname{Tr} \widehat{\mathrm{P}} e^{\int d \hat{\sigma} \mathbf{M}(\Gamma)}(\mathrm{NSNS}), \quad Z_{1}=\operatorname{Str} \widehat{\mathrm{P}} e^{\int d \hat{\sigma} \mathbf{M}(\Gamma)}(\mathrm{RR}) \\
& Z_{2}=\int[d \boldsymbol{\Gamma}] \exp \left\{\int d \widehat{\sigma}\left(\frac{1}{4} \boldsymbol{\Gamma} D \boldsymbol{\Gamma}+\mathbf{M}(\boldsymbol{\Gamma})\right)\right\}
\end{aligned}
$$

where $\Gamma=\left(\Gamma^{1}, \ldots, \Gamma^{n}\right)$ are gamma matrices and $\Gamma=\left(\Gamma^{1}, \ldots, \Gamma^{n}\right)$ are corresponding superfields. The normalization constant $\kappa$ is $\kappa=1$ or $\kappa=1 / \sqrt{2}$ when $n$ is even or odd, respectively. This statement was proved in [59] using the point splitting regularization. Our method is useful because of its manifestation of supersymmetry, although result should be regularization independent.

First we introduce fermionic $*$-product between two polynomials of classical fermion fields $\eta^{i}$ as

$$
A(\eta) * B(\eta)=\left.e^{-\frac{\partial}{\partial \eta_{1}^{i}} \frac{\partial}{\partial \eta_{2}^{i}}} A\left(\eta_{1}\right) B\left(\eta_{2}\right)\right|_{\eta^{i}=\eta_{1}^{i}=\eta_{2}^{i}}
$$

This corresponds to the algebra among anti-symmetrized polynomials of the gamma matrix $\Gamma^{i}$. Actually, we can easily see that $A(\eta) * B(\eta)$ corresponds to $A(\Gamma) B(\Gamma)$ as in the bosonic case, where contractions of the Gamma matrices $\left(\Gamma^{i}\right)^{2}=1$ is represented by the fermionic $*$-product. It is also seen that $\kappa \operatorname{Tr}(A(\Gamma))$ and $\operatorname{Str}(A(\Gamma))=$ $\kappa \operatorname{Tr}\left((-i)^{n / 2} \prod_{i=1}^{n} \Gamma^{i} A(\Gamma)\right)$ correspond to $\left.2^{n / 2} A(\eta)\right|_{\eta^{i}=0}$ and $(-2 i)^{n / 2} \int d \eta^{1} \cdots d \eta^{n} A(\eta)$, respectively. 
The propagator for $\boldsymbol{\Gamma}$ in the path integral (A.20) is given by the sign function and we have

$$
\begin{aligned}
& \int[d \boldsymbol{\Gamma}] e^{\frac{1}{4} \int d \hat{\sigma} \boldsymbol{\Gamma} D \boldsymbol{\Gamma}} \mathbf{M}(\boldsymbol{\Gamma})_{\hat{\sigma}_{1}} \cdots \mathbf{M}(\boldsymbol{\Gamma})_{\hat{\sigma}_{n}} \\
= & \left.(-2 i)^{n / 2} \int \prod_{i} d \eta_{0}^{i} e^{\frac{\delta}{\delta \Gamma} \cdot \Theta \frac{\delta}{\delta \Gamma}} \mathbf{M}(\boldsymbol{\Gamma})_{\hat{\sigma}_{1}} \cdots \mathbf{M}(\boldsymbol{\Gamma})_{\hat{\sigma}_{n}}\right|_{\boldsymbol{\Gamma}^{i}(\hat{\sigma})=\eta_{0}^{i}},
\end{aligned}
$$

for RR-sector and $\left.2^{n / 2} e^{\frac{\delta}{\delta \boldsymbol{\Gamma}} \cdot \Theta \frac{\delta}{\delta \boldsymbol{\Gamma}}} \mathbf{M}(\boldsymbol{\Gamma})_{\hat{\sigma}_{1}} \cdots \mathbf{M}(\boldsymbol{\Gamma})_{\hat{\sigma}_{n}}\right|_{\boldsymbol{\Gamma}^{i}(\hat{\sigma})=0}$ for NSNS-sector. Here we have defined

$$
\frac{\delta}{\delta \boldsymbol{\Gamma}} \cdot \Theta \frac{\delta}{\delta \boldsymbol{\Gamma}}=-\int d \widehat{\sigma}_{a} \int d \widehat{\sigma}_{b} \Theta\left(\widehat{\sigma}_{a b}\right)\left(\frac{\delta}{\delta \boldsymbol{\Gamma}\left(\widehat{\sigma}_{a}\right)} \frac{\delta}{\delta \boldsymbol{\Gamma}\left(\widehat{\sigma}_{b}\right)}\right) .
$$

From these we obtain $Z_{1}=Z_{2}$ repeating the argument for the bosonic case.

Combining these results, we can directly show the equivalence between path integral and operator formulation of the boundary interaction used in the paper.

\section{B Normalization}

In order to fix the normalization, we have to determine the path integral measure. We recall the equivalence between the operator formulation and the path integral formulation of a free particle;

$$
\operatorname{Tr}\left(e^{-i \int d \sigma \frac{\hat{p}^{2}}{2 m}}\right)=\int[d x] e^{i \int d \sigma \frac{m}{2} \dot{x}^{2}} .
$$

We use this equation to define the path integral measure for the bosonic variables. The left hand side of (B.1) can be evaluated as

$$
\operatorname{Tr}\left(e^{-2 \pi i \frac{\hat{p}^{2}}{2 m}}\right)=\int d p\left\langle p\left|e^{-2 \pi i \frac{\hat{p}^{2}}{2 m}}\right| p\right\rangle=\int d p e^{-2 \pi i \frac{p^{2}}{2 m}}\langle p \mid p\rangle=\frac{\sqrt{-i m}}{2 \pi} \int d x .
$$

On the other hand, the right hand side of (B.1) will become

$$
\begin{aligned}
\int[d x] e^{i \int d \sigma \frac{m}{2} \dot{x}^{2}} & =K \int d x_{0} \prod_{n=1}^{\infty} d x_{n} d x_{-n} e^{2 \pi i m \sum_{n=1}^{\infty} n x_{-n} x_{n}} \\
& =K \int d x_{0} \prod_{n=1}^{\infty} \frac{\pi}{-2 \pi i m n}=K \sqrt{\frac{-i m}{\pi}} \int d x_{0}
\end{aligned}
$$


where $K$ is a normalization constant. Here we have used the following formulae obtained by zeta function regularization;

$$
\prod_{n=1}^{\infty} A=A^{-1 / 2}, \quad \prod_{n=1}^{\infty} \frac{1}{n}=\frac{1}{\sqrt{2 \pi}} .
$$

In order to equate (B.2) and (B.4), we should set

$$
K=\frac{1}{2 \sqrt{\pi}}
$$

With this normalization, we can also show

$$
\begin{aligned}
\int[d p] e^{-i \int d \sigma \frac{1}{2 m} p^{2}} & =K \int d p_{0} e^{-\frac{\pi i}{m} p_{0}^{2}} \int \prod_{n=1}^{\infty} d p_{n} d p_{-n} e^{-\frac{2 \pi i}{m} \sum_{n=1}^{\infty} \frac{1}{n} p_{-n} p_{n}} \\
& =K \sqrt{-i m} \prod_{n=1}^{\infty} \frac{\pi m n}{2 \pi i}=K 2 \sqrt{\pi} \\
& =1
\end{aligned}
$$

which implies, as usual,

$$
\int[d x] e^{i \int d \sigma \frac{m}{2} \dot{x}^{2}}=\int[d x][d p] e^{i \int d \sigma\left(p \dot{x}-\frac{1}{2 m} p^{2}\right)}
$$

If we only perform the integral with respect to non-zero modes in the right hand side of (B.8), we obtain

$$
\begin{aligned}
& \int[d x][d p] e^{i \int d \sigma\left(p \dot{x}-\frac{1}{2 m} p^{2}\right)} \\
= & K^{2} \int d x_{0} d p_{0} e^{-\frac{\pi i}{m} p_{0}^{2}} \int \prod_{n=1}^{\infty} d x_{n} d x_{-n} d p_{n} d p_{-n} e^{2 \pi i \sum_{n=1}^{\infty}\left(i p_{n} x_{-n}-i p_{-n} x_{n}-\frac{1}{m n} p_{-n} p_{n}\right)} \\
= & K^{2} \prod_{n=1}^{\infty}\left(\frac{\pi m n}{2 \pi i} \frac{\pi}{-2 \pi i m n}\right) \int d x_{0} d p_{0} e^{-\frac{\pi i}{m} p_{0}^{2}} \\
= & \frac{1}{2 \pi} \int d x_{0} d p_{0} e^{-\frac{\pi i}{m} p_{0}^{2}}
\end{aligned}
$$

This normalization factor $1 / 2 \pi$ in (B.12) is the origin of the factor $1 / 2 \pi$ that we encountered in (A.8).

For the fermions, we can use the path integral representation of matrices explained around (2.22). Recall that $2 \times 2$ matrices can be expanded by Pauli matrices $\sigma^{1}$ and 
$\sigma^{2}$, and we can represent them by fermions $\eta^{1}$ and $\eta^{2}$ in the path integral. Then, for the NS-NS sector, we have

$$
\begin{aligned}
2 & =\operatorname{Tr}\left(\begin{array}{ll}
1 & \\
& 1
\end{array}\right)=\int\left[d \eta^{1}\right]\left[d \eta^{2}\right] e^{\int d \sigma \frac{1}{4}\left(\dot{\eta}^{1} \eta^{1}+\dot{\eta}^{2} \eta^{2}\right)} \\
& =\left(K_{\eta} \int \prod_{r=1 / 2}^{\infty} d \eta_{r}^{1} d \eta_{-r}^{1} e^{\sum_{r=1 / 2}^{\infty} i \pi r \eta_{-r}^{1} \eta_{r}^{1}}\right)^{2} \\
& =\left(K_{\eta} \prod_{r=1 / 2}^{\infty}(i \pi r)\right)^{2}=2 K_{\eta}^{2} .
\end{aligned}
$$

Therefore the normalization constant is $K_{\eta}=1$. Here we have used the following the zeta function regularization formulae;

$$
\prod_{r=1 / 2,3 / 2, \cdots}^{\infty} A=1, \quad \prod_{r=1 / 2,3 / 2, \cdots}^{\infty} r=\sqrt{2} .
$$

In particular, we obtain

$$
\int[d \eta] e^{\int d \sigma \frac{1}{4} \dot{\eta} \eta}=\sqrt{2}
$$

for the NS-NS sector boundary fermion. The analogous formula in superfield formulation is also useful;

$$
\int[d \boldsymbol{\Gamma}] e^{\int d \hat{\sigma} \frac{1}{4} \boldsymbol{\Gamma} D \boldsymbol{\Gamma}}=\int[d \eta][d F] e^{\int d \sigma \frac{1}{4}\left(\dot{\eta} \eta+F^{2}\right)}=\sqrt{2}
$$

where we have used the analytic continuation of the formula (B.7) for the integration with respect to the auxiliary field $F$.

Similarly, the normalization constant for the R-R sector is determined by

$$
\begin{aligned}
2 & =\operatorname{Str} \sigma^{3}=\int\left[d \eta^{1}\right]\left[d \eta^{2}\right] i \eta_{0}^{2} \eta_{0}^{1} e^{\int d \sigma \frac{1}{4}\left(\dot{\eta}^{1} \eta^{1}+\dot{\eta}^{2} \eta^{2}\right)} \\
& =i\left(K_{\eta} \int d \eta_{0}^{1} \eta_{0}^{1} \int \prod_{n=1}^{\infty} d \eta_{n}^{1} d \eta_{-n}^{1} e^{\sum_{n=1}^{\infty} i \pi n \eta_{-n}^{1} \eta_{n}^{1}}\right)^{2} \\
& =i\left(K_{\eta} \prod_{n=1}^{\infty}(i \pi n)\right)^{2}=2 K_{\eta}^{2},
\end{aligned}
$$

which again implies $K_{\eta}=1$. Thus we obtain

$$
\int[d \eta] \eta_{0} e^{\int d \sigma \frac{1}{4} \dot{\eta} \eta}=\sqrt{-2 i}
$$


for the R-R sector boundary fermion.

Using these definition of the path integral measures, we can fix the overall normalization of the $\mathrm{D} p$-brane action (2.40) as

$$
S^{D p}=\frac{2 \pi}{g_{s}} \int\left[d \mathbf{x}^{\alpha}\right]\left\langle 0\left|e^{-S_{b}}\right| \mathbf{x}^{\alpha}, \mathbf{x}^{i}=0 ;+\right\rangle_{\mathrm{NS}} .
$$

In fact, the tension for a BPS D $p$-brane is correctly obtained by turning off the fields on the $\mathrm{D} p$-brane;

$$
\begin{aligned}
& \frac{2 \pi}{g_{s}} \int\left[d \mathbf{x}^{\alpha}\right]\left\langle 0 \mid \mathbf{x}^{\alpha} ;+\right\rangle_{\mathrm{NS}} \\
= & \frac{2 \pi}{g_{s}} \prod_{\alpha=0}^{p}\left(K \int d x_{0}^{\alpha} \prod_{n=1}^{\infty} d x_{n}^{\alpha} d x_{-n}^{\alpha} \prod_{r=1 / 2}^{\infty} d \psi_{r}^{\alpha} d \psi_{-r}^{\alpha} e^{-\frac{1}{2} \sum_{n=1}^{\infty} x_{-n}^{\alpha} x_{n}^{\alpha}-\frac{1}{2} \sum_{r=1 / 2}^{\infty} \psi_{-r}^{\alpha} \psi_{r}^{\alpha}}\right) \\
= & \frac{2 \pi}{g_{s}}\left(\frac{1}{2 \sqrt{2} \pi}\right)^{p+1} \int d^{p+1} x_{0}=\mathcal{T}_{p} \int d^{p+1} x_{0},
\end{aligned}
$$

where

$$
\mathcal{T}_{p}=\frac{1}{(2 \pi)^{p}\left(\sqrt{\alpha^{\prime}}\right)^{p+1} g_{s}}
$$

is the tension for the $\mathrm{D} p$-brane with the convention $\alpha^{\prime}=2$. For the non-BPS $\mathrm{D} p$-branes, we have an additional $\sqrt{2}$ factor which comes from the contribution of the boundary fermion (B.17). It is important to note that the normalization constant $2 \pi / g_{s}$ in (B.23) is independent of $p$. Therefore, once we obtain the descent/ascent relations among the boundary states $|B p ;+\rangle_{S_{b}}$, we can exactly reproduce the $\mathrm{D} p$-brane action including the tension.

The coefficient for the CS-term $\mu_{p}$ in (3.74) is similarly determined as

$$
\begin{aligned}
\mu_{p} & =\prod_{\alpha=0}^{p}\left(K \int \prod_{n=1}^{\infty} d x_{n}^{\alpha} d x_{-n}^{\alpha} \prod_{n=1}^{\infty} d \psi_{n}^{\alpha} d \psi_{-n}^{\alpha} e^{-\frac{1}{2} \sum_{n=1}^{\infty} x_{-n}^{\alpha} x_{n}^{\alpha}-\frac{1}{2} \sum_{n=1}^{\infty} \psi_{-n}^{\alpha} \psi_{n}^{\alpha}}\right) \\
& =\frac{1}{(2 \pi)^{p+1}} .
\end{aligned}
$$

\section{A-roof genus in CS-term}

Here we summarize the computation to obtain the A-roof genus in the CS-term explained in section 3.6.3. We will show that if we include the scalar fields and perform 
the path integral in the $u \rightarrow \infty$ limit, we will recover the CS-term of the form (3.112), where $R$ is replaced by the curvature two form with respect to the induced metric.

When we include the scalar field, the boundary action (3.111) will become

$$
\begin{aligned}
S_{b} & =S_{g}+S_{A}+S_{\phi} \\
S_{g} & =\int d \sigma\left(\frac{1}{4 u^{2}} g_{\alpha \beta}(x)\left(\dot{x}^{\alpha} \dot{x}^{\beta}+\psi^{\alpha} \nabla_{\sigma} \psi^{\beta}\right)\right), \\
S_{A} & =\int d \sigma\left(A_{\alpha}(x) \dot{x}^{\alpha}-\frac{1}{2} F_{\alpha \beta}(x) \psi^{\alpha} \psi^{\beta}\right), \\
S_{\phi} & =\int d \sigma\left(i P_{\alpha} x^{\alpha}+i \Pi_{\alpha} \psi^{\alpha}+i P_{i} \phi^{i}(x)+i \Pi_{i} \partial_{\alpha} \phi^{i}(x) \psi^{\alpha}\right) .
\end{aligned}
$$

We split the fields $x$ and $\psi$ into zero modes $x_{0}, \psi_{0}$ and non-zero modes $\delta x, \delta \psi$ as

$$
x=x_{0}+\delta x, \quad \psi=\psi_{0}+\delta \psi
$$

and consider the path integral with respect to the non-zero modes. Note that the $S_{\phi}$ will act on the boundary state as

$$
\begin{aligned}
& e^{-S_{\phi}}\left|x^{\mu}=0\right\rangle\left|\psi^{\mu}=0\right\rangle_{\mathrm{RR}} \\
= & \left|x^{\alpha}, x^{i}=\phi^{i}(x)\right\rangle\left|\psi^{\alpha}, \psi^{i}=\partial_{\alpha} \phi^{i}(x) \psi^{\alpha}\right\rangle_{\mathrm{RR}} \\
= & e^{\sum_{m=1}^{\infty}\left\{-\frac{1}{\alpha^{\prime}}\left(\bar{g}_{\alpha \beta}\left(x_{0}\right)+\frac{1}{m} \bar{R}_{\alpha \beta}\left(x_{0}\right)\right) x_{-m}^{\alpha} x_{m}^{\beta}-\frac{1}{\alpha^{\prime}} \bar{g}_{\alpha \beta}\left(x_{0}\right) \psi_{-m}^{\alpha} \psi_{m}^{\beta}+\cdots\right\}}\left|x_{0}\right\rangle\left|\psi_{0}\right\rangle_{\mathrm{RR}},
\end{aligned}
$$

where $\bar{g}_{\alpha \beta}\left(x_{0}\right)=\delta_{\alpha \beta}+\partial_{\alpha} \phi^{i}\left(x_{0}\right) \partial_{\beta} \phi^{i}\left(x_{0}\right)$ is the induced metric and $\bar{R}_{\alpha \beta}\left(x_{0}\right)=\frac{1}{2} \bar{R}_{\alpha \beta \gamma \delta}\left(x_{0}\right) \psi_{0}^{\gamma} \psi_{0}^{\delta}$ is the curvature two form defined by the induced metric. Here we adopt the Riemann normal coordinates at $x_{0}$ (C.8) is obtained by inserting the expansions like

$$
\phi^{i}(x)=\phi^{i}\left(x_{0}\right)+\partial_{\alpha} \phi^{i}\left(x_{0}\right) \delta x^{\alpha}+\frac{1}{2} \partial_{\alpha} \partial_{\beta} \phi^{i}\left(x_{0}\right) \delta x^{\alpha} \delta x^{\beta}+\cdots
$$

into (2.6) and (2.7). In (C.8), we recovered the $\alpha^{\prime}$ dependence treating $\bar{g}_{\alpha \beta}$ as a dimensionless field.

Let us now consider the CS-term defined in (2.41) and we would like to perform the path integral with respect to the non-zero modes. We evaluate the Gaussian integral coming from (C.8) and consider other terms as perturbation. The contribution from $S_{g}$ in (C.2) can be dropped by taking the $u \rightarrow \infty$ limit. Then, the Gaussian integral 
will become exact in the $\alpha^{\prime} \rightarrow 0$ limit. As a result, we obtain the CS-term

$$
\begin{aligned}
S_{C S}^{D(-1)} & =C_{0} \mu_{p} \int d x_{0}^{\alpha} d \psi_{0}^{\alpha} \operatorname{Tr} e^{\pi F_{\alpha \beta} \psi_{0}^{\alpha} \psi_{0}^{\beta}} \prod_{m=1}^{\infty} \frac{1}{\operatorname{det}\left(1-\frac{1}{m} \bar{R}\right)} \\
& =C_{0} \int \operatorname{Tr} e^{F / 2 \pi} \wedge \widehat{A}(\bar{R})
\end{aligned}
$$

where $\bar{R}=\left(\bar{R}_{\beta}^{\alpha}\right)$. To obtain the A-roof genus in (C.11), we have used the formula

$$
\prod_{a} \prod_{m=1}^{\infty} \frac{1}{\left(1+\frac{\left(x_{a} / 2 \pi\right)^{2}}{m^{2}}\right)}=\prod_{a} \frac{x_{a} / 2}{\sinh \left(x_{a} / 2\right)}=\widehat{A}(\bar{R})
$$

where $x_{a}$ 's are the skew eigen values of the curvature two-form $\bar{R} / 2 \pi$.

Note also that if we consider $u \rightarrow 0$ limit with fixed $\alpha^{\prime}$, we will again recover (3.112) which is written in terms of the internal metric $g_{\alpha \beta}$.

Finally, we comment about the topological invariance of the CS-term coupled to $C_{0}$. Since the massless RR-state $|C\rangle$ does not contain higher level oscillators, the oscillator dependent part in (C.7) will drop in the computation of the CS-term. Then, the relevant part of (C.8) can be rewritten using superfields as

$$
\exp \left\{-\frac{1}{4} \int d \widehat{\sigma}\left(D \phi^{i}(\mathbf{x}) \frac{1}{\sqrt{D^{4}}} D^{2} \phi^{i}(\mathbf{x})\right)\right\}\left|x_{0}\right\rangle\left|\psi_{0}\right\rangle_{\mathrm{RR}}
$$

where $D^{2}=\partial_{\tau}$ and $\frac{1}{\sqrt{D^{4}}} D^{2}$ is well-defined non-local operator although $\frac{1}{\sqrt{D^{4}}}$ is not. Therefore, the coupling to the constant $C_{0}$ in the CS-term becomes the partition function of a supersymmetric theory, which can be rewritten as an index of the supercharge. This implies the topological invariance of the D-instanton charge. Note that the charge density is not topological invariant as we have seen above.

\section{References}

[1] J. Dai, R. G. Leigh and J. Polchinski, Mod. Phys. Lett. A 4 (1989) 2073.

[2] C. G. Callan, C. Lovelace, C. R. Nappi and S. A. Yost, "Adding Holes And Crosscaps To The Superstring," Nucl. Phys. B 293 (1987) 83; "Loop Corrections to Superstring Equations of Motion," Nucl. Phys. B 308 (1988) 221. 
[3] For a review, P. Di Vecchia and A. Liccardo, "D-branes in string theory, I," hep-th/9912161.

[4] T. Banks, W. Fischler, S.H. Shenker and L. Susskind, "M Theory as a Matrix Model: A Conjecture," Phys. Rev. 55 (1997) 5112, hep-th/9610043.

[5] A. Sen, "SO(32) Spinors of Type I and Other Solitons on Brane-Antibrane Pair," JHEP 9809 (1998) 023, hep-th/9808141;

A. Sen, "Non-BPS states and branes in string theory," hep-th/9904207, and references therein.

[6] R. Minasian and G. Moore, "K-theory and Ramond-Ramond charge," JHEP 9711 (1997) 002, hep-th/9710230.

[7] E. Witten, "D-branes and K-theory," JHEP 9812 (1998) 019, hep-th/9810188.

[8] P. Horava, "Type IIA D-Branes, K-Theory, and Matrix Theory," Adv. Theor. Math. Phys. 2 (1999) 1373, hep-th/9812135.

[9] S. Terashima, "A Construction of Commutative D-branes from Lower Dimensional Non-BPS D-branes," JHEP 0105 (2001) 059, hep-th/0101087.

[10] J. Kluson, "D-branes from N non-BPS D0-branes," JHEP 0011 (2000) 016, hepth/0009189.

[11] T. Asakawa, S. Sugimoto and S. Terashima, "D-branes, matrix theory and Khomology," JHEP 0203 (2002) 034, hep-th/0108085.

[12] K. Bardakci, "Dual Models and Spontaneous Symmetry Breaking," Nucl. Phys. B68 (1974) 331; K. Bardakci and M. B. Halpern, "Explicit Spontaneous Breakdown in a Dual Model," Phys. Rev. D10 (1974) 4230; K. Bardakci and M. B. Halpern, "Explicit Spontaneous Breakdown in a Dual Model II: N Point Functions," Nucl. Phys. B96 (1975) 285; K. Bardakci, "Spontaneous Symmetry Breakdown in the Standard Dual String Model," Nucl. Phys. B133 (1978) 297 ; 
[13] A. Sen, "BPS D-branes on non-supersymmetric cycles," JHEP 9812 (1998) 021, hep-th/9812031.

A. Sen, "Descent relations among bosonic D-branes," Int. J. Mod. Phys. A 14 (1999) 4061, hep-th/9902105.

J. Majumder and A. Sen, "Blowing up D-branes on Non-supersymmetric Cycles," JHEP 9909 (1999) 004, hep-th/9906109.

J. Majumder and A. Sen, "Vortex pair creation on brane-antibrane pair via marginal deformation," JHEP 0006 (2000) 010, hep-th/0003124.

M. Naka, T. Takayanagi and T. Uesugi, "Boundary state description of tachyon condensation," JHEP 0006 (2000) 007, hep-th/0005114.

[14] N. Ishibashi, " $p$-branes from $(p-2)$-branes in the Bosonic String Theory," Nucl. Phys. B539 (1999) 107, hep-th/9804163.

[15] E. Witten, "On background independent open string field theory," Phys. Rev. D 46 (1992) 5467, hep-th/9208027.

[16] A. A. Gerasimov and S. L. Shatashvili, "On exact tachyon potential in open string field theory," JHEP 0010 (2000) 034, hep-th/0009103.

[17] D. Kutasov, M. Marino and G. W. Moore, "Some exact results on tachyon condensation in string field theory," JHEP 0010 (2000) 045, hep-th/0009148.

[18] D. Kutasov, M. Marino and G. Moore, "Remarks on tachyon condensation in superstring field theory," hep-th/0010108.

[19] A.A. Tseytlin, "Sigma model approach to string theory effective actions with tachyons," J. Math. Phys. 42 (2001) 2854, hep-th/0011033.

[20] P. Kraus and F. Larsen, "Boundary String Field Theory of the DDbar System," Phys. Rev. D63 (2001) 106004, hep-th/0012198.

[21] T. Takayanagi, S. Terashima and T. Uesugi, "Brane-Antibrane Action from Boundary String Field Theory," JHEP 0003 (2001) 019, hep-th/0012210. 
[22] S.P. de Alwis, "Boundary String Field Theory, the Boundary State Formalism and D-Brane Tension," Phys. Lett. B505 (2001) 215, hep-th/0101200.

[23] O. D. Andreev and A. A. Tseytlin, "Partition Function Representation For The Open Superstring Effective Action: Cancellation Of Mobius Infinities And Derivative Corrections To Born-Infeld Lagrangian," Nucl. Phys. B 311 (1988) 205.

[24] A. Connes, "Noncommutative geometry," Academic Press, 1994. See also,

A. Connes, "A Short Survey of Noncommutative Geometry," hep-th/0003006.

A. Connes, "Noncommutative Geometry Year 2000," math.QA/0011193.

[25] T. Asakawa, S. Sugimoto and S. Terashima, "D-branes and KK-theory in type I string theory," JHEP 0205 (2002) 007, hep-th/0202165.

[26] J. A. Harvey, D. Kutasov and E. J. Martinec, "On the relevance of tachyons," hep-th/0003101.

[27] N. Wyllard, "Derivative corrections to D-brane actions with constant background fields," Nucl. Phys. B598 (2001) 247, hep-th/0008125.

[28] P. Di Vecchia, M. Frau, A. Lerda, A. Liccardo, “(F,D $p)$ bound states from the boundary state." Nucl. Phys. B 565 (2000) 397, hep-th/9906214.

[29] S. Terashima and T. Uesugi, "On the Supersymmetry of Non-BPS D-brane," JHEP 0105 (2001) 054, hep-th/0104176.

[30] Y. Okawa and H. Ooguri, "An Exact Solution to Seiberg-Witten Equation of Noncommutative Gauge Theory," Phys. Rev. D64 (2001) 046009, hep-th/0104036.

[31] S. Mukhi and N. V. Suryanarayana, "Gauge-invariant couplings of noncommutative branes to Ramond-Ramond backgrounds," JHEP 0105 (2001) 023, hepth/0104045. 
[32] H. Liu and J. Michelson, "Ramond-Ramond couplings of noncommutative Dbranes," Phys. Lett. B 518 (2001) 143, hep-th/0104139.

[33] M. Billo, P. Di Vecchia, M. Frau, A. Lerda, I. Pesando, R. Russo and S. Sciuto, "Microscopic string analysis of the D0-D8 brane system and dual R-R states," Nucl. Phys. B 526 (1998) 199, hep-th/9802088.

[34] C. Kennedy and A. Wilkins, Phys. Lett. B 464 (1999) 206, hep-th/9905195.

[35] D. Quillen, "Superconnection and the Chern character," Topology 24 (1985) 89.

[36] R.C. Myers, "Dielectric-Branes," JHEP 9912 (1999) 022, hep-th/9910053.

[37] M. F. Atiyah and I. M. Singer, "The index of elliptic operators I, III," Ann. of Math. 87 (1968), 484-530, 546-604.

[38] L. Alvarez-Gaume, "Supersymmetry And The Atiyah-Singer Index Theorem," Commun. Math. Phys. 90 (1983) 161.

[39] D. Friedan and P. Windey, "Supersymmetric Derivation Of The Atiyah-Singer Index And The Chiral Anomaly," Nucl. Phys. B 235 (1984) 395.

[40] M. B. Green, J. A. Harvey and G. W. Moore, "I-brane inflow and anomalous couplings on D-branes," Class. Quant. Grav. 14 (1997) 47, hep-th/9605033. Y. K. Cheung and Z. Yin, "Anomalies, branes, and currents," Nucl. Phys. B 517 (1998) 69, hep-th/9710206.

[41] A. Alekseev and V. Schomerus, "RR charges of D2-branes in the WZW model," hep-th/0007096.

[42] N. Ishibashi, "A relation between commutative and noncommutative descriptions of D-branes," hep-th/9909176.

[43] L. Cornalba, "D-brane physics and noncommutative Yang-Mills theory," Adv. Theor. Math. Phys. 4 (2000) 271, hep-th/9909081. 
[44] K. Okuyama, "Boundary States in B-Field Background," Phys. Lett. B499 (2001) 305, hep-th/0009215.

[45] K. Okuyama, "A Path Integral Representation of the Map between Commutative and Noncommutative Gauge Fields," JHEP 0003 (2000) 016, hep-th/9910138.

[46] N. Seiberg and E. Witten, JHEP 9909 (1999) 032, hep-th/9908142.

[47] E. T. Akhmedov, A. A. Gerasimov and S. L. Shatashvili, "On unification of RR couplings," JHEP 0107 (2001) 040, hep-th/0105228.

[48] M. Frau, L. Gallot, A. Lerda and P. Strigazzi, "Stable non-BPS D-Branes in Type I String Theory," Nucl. Phys. B564 (2000) 60, hep-th/9903123;

M. Frau, L. Gallot, A. Lerda and P. Strigazzi, "Stable non-BPS D-Branes of Type I," hep-th/0003022.

[49] O. Bergman, "Tachyon condensation in unstable type I D-brane systems," JHEP 0011 (2000) 015, hep-th/0009252.

[50] S. Sugimoto and S. Terashima, "Tachyon matter in boundary string field theory," JHEP 0207 (2002) 025, hep-th/0205085.

[51] J. A. Minahan, "Rolling the tachyon in super BSFT," JHEP 0207 (2002) 030, hep-th/0205098.

[52] P. Yi, "Membranes from five-branes and fundamental strings from Dp branes," Nucl. Phys. B 550 (1999) 214, hep-th/9901159.

[53] O. Bergman, K. Hori and P. Yi, "Confinement on the brane," Nucl. Phys. B 580 (2000) 289, hep-th/0002223.

[54] J. A. Harvey, P. Kraus, F. Larsen and E. J. Martinec, "D-branes and strings as non-commutative solitons," JHEP 0007 (2000) 042, hep-th/0005031.

[55] A. Sen, "Supersymmetric World-volume Action for Non-BPS D-branes," JHEP 9910 (1999) 008, hep-th/9909062. 
[56] T. Yoneya, "Spontaneously Broken Space-Time Supersymmetry in Open String Theory without GSO Projection," Nucl. Phys. B576 (2000) 219, hep-th/9912255.

[57] K. Li and E. Witten, "Role of short distance behavior in off-shell open string field theory," Phys. Rev. D 48 (1993) 853, hep-th/9303067.

[58] H. Aoki, N. Ishibashi, S. Iso, H. Kawai, Y. Kitazawa and T. Tada, "Noncommutative Yang-Mills in IIB Matrix Model," Nucl. Phys. B 565 (2000) 176, hepth/9908141.

[59] N. Marcus, "Open String And Superstring Sigma Models With Boundary Fermions," DOE-ER-40423-09-P8. 